\title{
NON-HURWITZ CLASSICAL GROUPS
}

\author{
R. VINCENT AND A.E. ZALESSKI
}

\begin{abstract}
In previous work by Di Martino, Tamburini and Zalesski [Comm. Algebra 28 (2000) 5383-5404] it is shown that certain low-dimensional classical groups over finite fields are not Hurwitz. In this paper the list is extended by adding the special linear and special unitary groups in dimensions $8,9,11,13$. We also show that all groups $\operatorname{Sp}(n, q)$ are not Hurwitz for $q$ even and $n=6,8,12,16$. In the range $11<n<32$ many of these groups are shown to be non-Hurwitz. In addition, we observe that $\operatorname{PSp}(6,3), P \Omega^{ \pm}\left(8,3^{k}\right), P \Omega^{ \pm}(10, q), \Omega\left(11,3^{k}\right)$, $\Omega^{ \pm}\left(14,3^{k}\right), \Omega^{ \pm}\left(16,7^{k}\right), \Omega\left(n, 7^{k}\right)$ for $n=9,11,13, \operatorname{PSp}\left(8,7^{k}\right)$ are not Hurwitz.
\end{abstract}

\section{Introduction}

A finite group $H \neq 1$ is called Hurwitz if it is generated by two elements $X, Y$ satisfying the conditions $X^{2}=Y^{3}=(X Y)^{7}=1$. A long-standing problem is that of classifying simple Hurwitz groups. The problem has been solved for alternating groups by Conder [4], and for sporadic groups by several authors with the latest result by Wilson [27]. It remains open for groups of Lie type and for classical groups. Quite a lot is known. Groups ${ }^{3} D_{4}(q)$ for $(q, 3)=1,{ }^{2} G_{2}(q), G_{2}(q)$ are Hurwitz with few exceptions, groups ${ }^{3} D_{4}\left(3^{k}\right)$ are not Hurwitz; see Jones [10] and Malle [15, 16]. Classical groups of large rank are Hurwitz; see [12], [13] and [26]. However many classical groups of small rank are not Hurwitz [6]. The current state of the problem and its history is discussed in a survey of Tamburini and Vsemirnov [23]. Formally, the problems of determining all Hurwitz groups and all non-Hurwitz groups are equivalent. However, proving that a given group is Hurwitz or non-Hurwitz requires very different technique. In this paper we focus on proving that certain groups are not Hurwitz.

We show that $\operatorname{Sp}(6, q), \operatorname{Sp}(8, q), \operatorname{Sp}(10, q), \operatorname{Sp}(12, q)$ and $\operatorname{Sp}(16, q)$ with $q$ even are not Hurwitz groups. In addition, groups $\operatorname{PSp}(6,3), P \Omega^{ \pm}\left(8,3^{k}\right), P \Omega^{ \pm}(10, q)$, $\Omega\left(11,3^{k}\right), \Omega^{ \pm}\left(14,3^{k}\right), \Omega^{ \pm}\left(16,7^{k}\right), \Omega\left(n, 7^{k}\right)$ for $n=9,11,13, \operatorname{PSp}\left(8,7^{k}\right)$ are not Hurwitz. We extend the results of $[6]$ by proving that groups $\operatorname{SL}(n, q)$ and $\operatorname{SU}(n, q)$ are not Hurwitz for $n=8,9,11,13$. If $n$ is coprime to $q-1$ then $\operatorname{SL}(n, q)$ is simple. Similarly, $\mathrm{SU}(n, q)$ is simple if $n$ is coprime to $q+1$. Therefore, these results contribute to the above problem. In addition, we show that for $n \in\{12,14,15,16,17,18,19,22,23$, $24,25,31\}$ there are infinitely many values of $q$ such that $\operatorname{SL}(n, q)$ is not $\operatorname{Hurwitz}$ and there are infinitely many values of $q$ such that $\mathrm{SU}(n, q)$ is not Hurwitz; see Table 1.

Received 30 August 2005, revised 11 March 2006; published 15 March 2007. 2000 Mathematics Subject Classification 20C99, 20D05, 20F38, 20H30, 30F10.

(C) 2007, R. Vincent and A.E. Zalesski 
TheOREM 1.1. (1) Groups $\mathrm{SL}(n, q)$ and $\mathrm{SU}(n, q)$ are not Hurwitz if $n<14$ and $n \neq 12$ except for $\mathrm{SL}(2,8)$ and $\mathrm{SL}(3,2)$. In addition, groups $\operatorname{SL}(n, q)$ and $\mathrm{SU}(n, q)$ are not Hurwitz if $q \equiv 0(\bmod 3)$ and $n=14$ and $q \equiv 0(\bmod 7)$ and $n=12,17,18$.

(2) Groups $\operatorname{Sp}(n, q)$ with $q$ even are not Hurwitz if $n=6,8,10,12$ and $n=16$.

Let $H_{237}$ be the group defined by two generators $x, y$ subject to relations $x^{2}=$ $y^{3}=(x y)^{7}$. Theorem 1.1 follows from our more general results on representations of $H_{237}$. The first is the following (where o denotes a central product).

THEOREM 1.2. Let $F$ be an algebraically closed field of characteristic $p>0$ and set $H=H_{237}$. Let $\phi: H \rightarrow \mathrm{GL}(n, F)$ be an irreducible representation such that $G=\phi(H)$ preserves no symmetric bilinear form on $V=F^{n}$. Define $\bar{p}=p$ if $\left(7, p^{3}-p\right) \neq 1$ and $\bar{p}=p^{3}$ otherwise.

(A) Suppose that $p \neq 2,3,7$. Then one of the following holds:

(A1) $n>13$ or $n=12$;

(A2) $n=3$ and $G \cong \mathrm{SL}(3,2)$;

(A3) $n=8$ and $G \cong \operatorname{SL}(2,7) \circ \operatorname{SL}(2, \bar{p})$;

(A4) $n=9$ and $G \cong \operatorname{SL}(3,2) \times \operatorname{PSL}(2, \bar{p})$;

(A5) $n=13$ and $G \cong \operatorname{PSL}(2,27)$.

(B) Suppose that $p=2$. Then one of the following holds:

(B1) $n>13$ or $n=12$;

(B2) $n=3$ and $G \cong \operatorname{SL}(3,2)$;

(B3) $n=6$ and $G \cong \operatorname{SL}(3,2) \times \operatorname{SL}(2,8)$;

(B4) $n=13$ and $G \cong \operatorname{PSL}(2,27)$.

(C) Suppose that $p=3$. Then one of the following holds:

(C1) $n>14$ or $n=12$;

(C2) $n=3$ and $G \cong \operatorname{SL}(3,2)$;

(C3) $n=8$ and $G \cong \mathrm{SL}(2,7) \circ \mathrm{SL}(2,27)$;

(C4) $n=9$ and $G \cong \operatorname{SL}(3,2) \times \operatorname{PSL}(2,27)$.

(D) Suppose that $p=7$. Then one of the following holds:

(D1) $n>13$ and $n \neq 17,18$;

(D2) $n=13$ and $G \cong \operatorname{PSL}(2,27)$.

In particular, for $3<n<12$ and $n=13$ neither $\mathrm{SL}(n, q)$ nor $\mathrm{SU}(n, q)$ is Hurwitz. If $n \leqslant 7$ or $n=10$, these results are not new; see [3], [6] and [23].

In the next theorem we assume $n>13$ as the cases $n \leqslant 13, n \neq 12$ are considered in Theorem 1.2, and for $n=12$ our computations do not extend the results of [6].

TheOREM 1.3. Assume that $n>13$. Then for the values of $(n, q)$ given in Tables 1 and 2 groups $\mathrm{SL}(n, q)$ and $\mathrm{SU}(n, q)$ are not Hurwitz.

More generally, let $\rho: H_{237} \rightarrow \mathrm{GL}(n, q)$ (respectively, $H_{237} \rightarrow U(n, q)$ ) be an absolutely irreducible representation and $G:=\rho(H)$. If $(n, q)$ appears at the 2 nd column of Table 1 or the 3 rd column of Table 2, then $G$ preserves a symmetric bilinear form. 
Theorem 1.4. Let $H=H_{237}$ and $\phi: H \rightarrow \operatorname{Sp}(n, q)$ be an absolutely irreducible representation.

(1) If $p \neq 2$ then $n>20$ and $n \neq 22$. In addition, if $q \not \equiv 0, \pm 1(\bmod 7)$ then $n \neq 24$.

(2) Let $p=2$ and $6<n<18$. Then $n \neq 10$. If $n \in\{8,12,16\}$ then $\phi(H)$ preserves a quadratic form. In particular, groups $\operatorname{Sp}(n, q)$ with $q$ even and $n=$ 8,10,12,16 are not Hurwitz.

(3) Groups $\operatorname{Sp}(6, q)$ and $\Omega^{ \pm}(10, q)$ are not Hurwitz for $q$ even.

(4) Assume $p=2$ and $q \not \equiv 1(\bmod 7)$. Then $n \neq 12,16$, in particular, groups $\Omega^{ \pm}(12, q)$ and $\Omega^{ \pm}(16, q)$ are not Hurwitz. If $n=18,24$ then $\phi(H)$ preserves a quadratic form. In particular, groups $\operatorname{Sp}(18, q), \operatorname{Sp}(24, q)$ are not Hurwitz.

TheOREM 1.5. Let $p \neq 2, H=H_{237}$ and $\phi: H \rightarrow O(n, F)$ be an irreducible representation. Then $n \neq 10$. In addition, if $p=3$ then $n \neq 8,11,14,17$ and if $p=7$ then $n \neq 9,11,13,16,18$. In particular, the corresponding groups $\Omega(n, q)$ and $\Omega^{ \pm}(n, q)$ are not Hurwitz.

Theorem 1.5 for $p=3$ implies that groups ${ }^{3} D_{4}\left(3^{k}\right)$ are not Hurwitz which provides a new proof of this fact known from Malle [16].

Corollary 1.6. All groups $\operatorname{PSp}\left(8,7^{k}\right), P \Omega^{+}\left(8,3^{k}\right)$ and $P \Omega^{+}(10, q)$ are not Hurwitz.

Theorem 1.7. Let $p \neq 2,7, H=H_{237}$ and $\phi: H \rightarrow O(n, q)$ be an absolutely irreducible representation.

(1) If $q \not \equiv \pm 1(\bmod 7)$ then $n \neq 9,11,16,17,18,24$. In particular, for these $q$ groups $\Omega(n, q)$ are not Hurwitz for $n=9,11,17$ as well as $\Omega^{ \pm}(n, q)$ for $n=$ $16,18,24$.

(2) If $q \equiv 0(\bmod 3)$ and $q \neq 3^{3 k}$ then $n \neq 16,18,23,24$. In particular, for these $q$ groups $\Omega(n, q)$ for $n=9,11,23$ are not Hurwitz as well as $\Omega^{ \pm}(16, q)$ and $\Omega^{ \pm}(24, q)$.

Observe that the occurrence of certain values of $n$ in the boxes of Tables 1 and 2 is a consequence of results obtained in [6], especially, the boxes with $q \equiv$ $-1(\bmod 3)$ at the $\mathrm{SL}(n, q)$-column and with $q \equiv 1(\bmod 3)$ at the $\mathrm{SU}(n, q)$-column. Our computations have been performed independently and therefore confirm the results of [6]. The case $n=12$ known from [6] is included into the tables for reader's convenience.

We expect that our results concerning groups $\mathrm{SL}(n, q), \mathrm{SU}(n, q)$ and $\operatorname{Sp}(n, q)$ are close to being final (but not for the simple quotients of these groups). According to Table 1 the maximum value of $n$ for which some group $\operatorname{SL}(n, q)$ or $\mathrm{SU}(n, q)$ is not Hurwitz equals 31 (and equals 38 for $\mathrm{SU}(n, q)$ with $q$ even). In the opposite direction, in [26] it is shown that all groups $\mathrm{SL}(n, q)$ are Hurwitz for $n=49,57,63,64,70,77,85$ and many other with $n>90$; see also [28]. This is an additional evidence that small $n$, say for $n<39$, cannot be treated uniformly.

Let $\mathbf{Z}$ denote the ring of integers. Lucchini, Tamburini and Wilson [12] prove that all groups $\mathrm{SL}(n, \mathbf{Z})$ with $n>287$ are quotients of $H_{237}$. Vsemirnov [26] and Yongzhong Sun [28] extend this result for many other values of $n>48$. We have the following result:

TheOREM 1.8. Groups $G=\mathrm{SL}(n, \mathbf{Z})$ are not $(2,3,7)$-generated for $n \in\{22,23$, $24,25,26,29,30,31,32,37,38\}$ and $n \leqslant 20$. 
Table 1: Values of $n>11, n \neq 13$ for which $\mathrm{SL}(n, q), \mathrm{SU}(n, q)$ or $\operatorname{Sp}(n, q)$ with $q$ odd are not Hurwitz.

\begin{tabular}{|l|l|l|l|}
\hline \multicolumn{1}{|c|}{$q$} & \multicolumn{1}{|c|}{$\mathrm{SL}(n, q)$} & \multicolumn{1}{|c|}{$\mathrm{SU}(n, q)$} & $\begin{array}{l}\mathrm{Sp}(n, q) \\
n>18, \\
n \neq 22\end{array}$ \\
\hline$q \equiv 1(\bmod 21)$ & & $\leqslant 19,22$ & \\
\hline$q \equiv-1(\bmod 21)$ & $\leqslant 19,22$ & & \\
\hline$q \equiv 2,-10(\bmod 21)$ & $\leqslant 19,23$ & $12,16,17,18,23,24$ & 24 \\
\hline$q \equiv-2,10(\bmod 21)$ & $12,16,17,18,23,24$ & $\leqslant 19,23$ & 24 \\
\hline$q \equiv 4,-5(\bmod 21)$ & 12,23 & $\leqslant 19,22,23,24,25,31$ & 24 \\
\hline$q \equiv-4,5(\bmod 21)$ & $\leqslant 19,22,23,24$, & 12,23 & 24 \\
& 25,31 & & \\
\hline$q \equiv 8(\bmod 21)$ & 14 & $12,16,17,18$ & \\
\hline$q \equiv-8(\bmod 21)$ & $12,16,17,18$ & 14 & \\
\hline$q=3^{6 k}$ & 14 & $\leqslant 19,22$ & 24 \\
\hline$q=3^{6 k+3}$ & $\leqslant 19,22$ & 14 & 24 \\
\hline$q=3^{6 k \pm 1}$ & $\leqslant 19,22,23,24$, & $\leqslant 16,23,25$ & \\
\hline$q=3^{6 k \pm 2}$ & 25,31 & $\leqslant 16,23,25$ & $\leqslant 19,22,23,24,25,31$ \\
\hline$q \equiv 0(\bmod 7)$ & $\leqslant 12,17,18$ & $\leqslant 19,22$ & 24 \\
\hline
\end{tabular}

Table 2: Values of $n>11, n \neq 13$ for which $\mathrm{SL}(n, q), \mathrm{SU}(n, q)$ or $\mathrm{Sp}(n, q)$ with $q$ even are not Hurwitz.

\begin{tabular}{|l|l|l|l|}
\hline$q$ & \multicolumn{1}{|c|}{$\mathrm{SL}(n, q)$} & \multicolumn{1}{|c|}{$\mathrm{SU}(n, q)$} & $\mathrm{Sp}(n, q)$ \\
\hline $2^{6 k}$ & & $\leqslant 20,22,23$ & 12,16 \\
\hline $2^{6 k \pm 1}$ & $\leqslant 17,19,22,23,24,25$ & $16,17,18,19,23,24,31$ & $12,16,18,24$ \\
\hline $2^{6 k \pm 2}$ & $16,17,19,22,23$ & $\leqslant 20,22,23,24,25,26,30$, & $12,16,18,24$ \\
& & $31,32,38$ & \\
\hline $2^{6 k+3}$ & 14 & $16,17,18,19,23,24$ & 12,16 \\
\hline
\end{tabular}

As in [6], our method is based on a theorem of Scott [19, page 491] (see Theorem 2.1 below). Scott himself pointed out that his result can be used for showing that certain linear groups are not Hurwitz (he provided examples of $\mathrm{SL}(6,3)$ and $\mathrm{SL}(9,3))$. In a more systematic way Theorem 2.1 was used in Tamburini and Vassallo [22] and in Di Martino, Tamburini and Zalesski [6]. In particular, it was shown in [6] that the groups $\mathrm{SL}(n, q)$ and $\mathrm{SU}(n, q)$ with $n=5,6,7,10$ are not Hurwitz; however, the potential of Scott's theorem was not used in full. In this paper we examine further values of $n$.

Our method can be outlined as follows. To show that some group $H$ which contains non-central elements of order 2,3,7 is not Hurwitz, one could first realize $H$ as an irreducible matrix group over some algebraically closed field $F$ obtaining an $F H$-module $M$, say. If $H$ is Hurwitz with Hurwitz generators $X, Y$, Scott's formula 
in Theorem 2.1 provides an essential restriction to the shape of the Jordan normal form of matrices corresponding to $X, Y$ and $X Y$. Usually, one chooses $M$ to be a non-trivial module of minimum dimension. However, applying Scott's theorem solely to $M$ does not lead to major progress. Other useful modules to be examined are the adjoint module $\operatorname{Hom}(M, M)$ and the symmetric square of $M$. These have been used in Di Martino, Tamburini and Zalesski [6] to show that the groups $\mathrm{SL}(n, q)$ and $\mathrm{SU}(n, q)$ are not Hurwitz for $n=5,6,7,10$. We observe here that, instead of dealing with an individual finite group $H$, it is beneficial to deal with representations of the infinite group $H_{237}$ defined by two generators $x, y$ subject to relations $x^{2}=y^{3}=(x y)^{7}$. If $H$ is Hurwitz, there is a surjective homomorphism $H_{237} \rightarrow H$ so every $F H$-module can be viewed as an $F H_{237}$-module. The original question of whether $H=\mathrm{SL}(n, q)$ or $\mathrm{SU}(n, q)$ is Hurwitz can be replaced by the question on the existence of an irreducible representation $\phi: H_{237} \rightarrow \mathrm{GL}(n, F)$ such that $\phi\left(H_{237}\right)$ does not preserve a symmetric non-degenerate bilinear form, where $F$ contains the field of $q$ elements. (If $q$ is odd, this is equivalent to saying that $H$ is not a subgroup of an orthogonal group.) Scott's formula applies equally to $\phi\left(H_{237}\right)$ but now we have a larger store of modules. For instance there is an irreducible $\mathrm{FH}_{237^{-}}$ module $L$ of dimension 3 while there is no irreducible 3 -dimensional $\operatorname{SL}(n, q)$-module for $n>3$. This allows us to apply Scott's formula to $F H_{237}$-module $\operatorname{Hom}(M, L)$. Surprisingly, this eliminates certain options for the conjugacy class choice for the Hurwitz generators in $H=\mathrm{SL}(n, q)$ and $H=\mathrm{SU}(n, q)$ with $n=8,9,11,13$, and leads to the conclusion that these $H$ are not Hurwitz.

Let $H$ be as above and $Z(H)$ the center of $H$. It has to be noticed that the group $H / Z(H)$ can be Hurwitz but $H$ itself is not. Therefore, one is faced with the problem of deciding which projective groups $\operatorname{PSL}(n, q)$ and $\operatorname{PSU}(n, q)$ are Hurwitz provided $H=\mathrm{SL}(n, q)$ and $\mathrm{SU}(n, q)$ are not Hurwitz. This happens for $n=2$ as no $\mathrm{SL}(2, q)$ with $q$ odd is Hurwitz. Another series of examples is discovered in [25] for $n=5$. Further results on $\operatorname{PSL}(n, q)$ and $\operatorname{PSU}(n, q)$ for $n \leqslant 7$ are obtained in a recent paper [24] (which also contains a few auxiliary facts recorded in Section 2 below).

In Section 2 we describe the method in detail. In Section 3 we list a few irreducible $\mathrm{FH}_{237}$-modules that are particularly useful in our analysis.

In Section 4 we discuss tests for an irreducible $F H_{237}$-module $V$ arising in applying Scott's theorem to the adjoint module $V \otimes \hat{V}$ as well as to the symmetric and exterior square of $V$ (which are submodules of $V \otimes V$ ).

Section 5 contains proofs of the theorems stated in the introduction.

Notation. $F$ always denotes an algebraically closed field of characteristic $p \geqslant 0$. We denote by $M(n, F)$ the vector space of $(n \times n)$-matrices over $F$ and by $\operatorname{GL}(n, F)$ the group of all non-degenerate matrices. $\operatorname{SL}(n, F)$ is the $\operatorname{subgroup}$ of $\operatorname{GL}(n, F)$ of matrices of determinant 1 . The identity $(n \times n)$-matrix will be denoted by Id or $1_{n}$. We denote by $S$ and $E$ the subspaces of $M(n, F)$ constituted by all symmetric matrices and all alternating matrices, respectively; an alternating matrix is the same as skew symmetric if $p \neq 2$ and as symmetric with zero diagonal if $p=2$. Let $V=F^{n}$ denote the natural $M(n, F)$-module. We use the standard notation for classical groups. If $A \subset \mathrm{GL}(V)$ is a subset then $V^{A}$ denotes the subspace of all vectors fixed by every element of $A$, and $d^{A}$ or $d_{V}^{A}$ is the dimension of $V^{A}$. We also set $C(A)=\{M \in M(n, F): M a=a M$ for all $a \in A\}$ and $c_{V}^{A}=\operatorname{dim} C(A)$. 
We use the symbol $\hat{V}$ for the dual GL(V)-module, and we set $\hat{d}_{V}^{A}=\operatorname{dim} \hat{V}^{A}$. This is also used in the representation context: if $\phi: G \rightarrow \mathrm{GL}(V)$ is a representation of a group $G$ and $A \subseteq G$ then $C(A)$ means $C(\phi(A))$ and the symbols $d^{\phi(A)}, d_{V}^{A}$ and $c_{V}^{A}$ carry the obvious meaning. If $V$ is an $F G$-module then the $F G$-module $\operatorname{Hom}(V, V) \cong V \otimes \hat{V}$ is called the adjoint module of $V$. The symmetric and exterior square of $V$ is often denoted by $S(V)$ and $E(V)$.

For a matrix $t$ we denote by Jord $t$ the Jordan form of $t$. The Jordan block of size $r$ with eigenvalue 1 is denoted by $J_{r}$. We set $k J_{r}=\operatorname{diag}\left(J_{r}, \ldots, J_{r}\right) \quad(k$ times). We often use friendly notation for Jord $A$ for a unipotent matrix $A$, namely, $\operatorname{diag}\left(k_{1} J_{1}, k_{2} J_{2}, \ldots, k_{\ell} J_{\ell}\right)$ where $k_{i}$ is the multiplicity of $J_{i}$ occurring as a constituent of Jord $A$. Say, $\operatorname{diag}\left(2 J_{1}, 2 J_{3}, J_{4}\right)$ means the block-diagonal matrix with Jordan blocks $1,1, J_{3}, J_{3}, J_{4}$ at the diagonal. An element $t \in \operatorname{GL}(n, F)$ is called real if it is conjugate in $\operatorname{GL}(n, F)$ to its inverse. Next we introduce a parametrization of certain conjugacy classes of $\mathrm{GL}(n, F)$ in terms of multiplicity vectors. This is useful for producing tables and performing computations.

Let $A$ be a diagonalizable matrix such that $A^{l}=$ Id for some $l$. In this case we have a natural ordering of the eigenvalues. If we fix a primitive $l$-root $\varepsilon$ of 1 , then we order other $l$-roots as follows: $1=\varepsilon^{0}, \varepsilon, \varepsilon^{2}, \ldots, \varepsilon^{l-1}$. Another choice of $\varepsilon$ means the replacement $\varepsilon \rightarrow \varepsilon^{i}$ where $i$ is coprime to $l$, but we need $\varepsilon$ to be fixed. If $F$ is the field of complex numbers then we always fix $\varepsilon$ to be $\exp (2 \pi i / l)$ where $i^{2}=-1$. The Jordan normal form of $A$ is determined by the string $\left[m_{0}, m_{1}, \ldots, m_{l-1}\right]$ where $m_{i}$ is the multiplicity of $\varepsilon$ as an eigenvalue of $A$ and some $m_{i}$ may be equal to 0 . We call the string $\left[m_{0}, m_{1}, \ldots, m_{l-1}\right]$ the multiplicity vector of $A$.

If $A$ is unipotent then Jord $A$ is determined by the sizes of the Jordan blocks. However, in some situations it is useful to parametrize unipotent matrices by multiplicity vectors. To do this, set $m_{i}=\operatorname{dim}(A-\mathrm{Id})^{i} V-\operatorname{dim}(A-\mathrm{Id})^{i+1} V$ where $V=F^{n}$ is the natural space for $A$. Clearly, $\sum m_{i}=n$. Observe that $m_{0}=\operatorname{dim} V-\operatorname{dim}(A-\mathrm{Id}) V$ and $m_{i}=\operatorname{rank}(A-\mathrm{Id})^{i}-\operatorname{rank}(A-\mathrm{Id})^{i+1}$ for $i>0$. The string $\left[m_{0}, m_{1}, \ldots, m_{l-1}\right]$ is called the multiplicity vector of $A$. One can check that $m_{i}=\sum_{j=i+1}^{l} k_{j}$. Observe that $m_{i}=0$ if $i$ is greater than the degree of the minimum polynomial of $A$. It is rather obvious that $m_{0} \geqslant m_{1} \geqslant \cdots \geqslant m_{l-1}$ if $A$ is unipotent. One can observe that a unipotent matrix is determined by the very values $\left\{m_{0}, \ldots, m_{l-1}\right\}$ as their ordering is immaterial (in the sense that the values can always be reordered to be non-increasing). This allows us to ignore the condition $m_{0} \geqslant \cdots \geqslant m_{l-1}$ for the coordinates of the multiplicity vector of a unipotent matrix. This will be used for uniformity purposes. For instance, the vector $[1,2]$ can be used as a label of the similarity class of the matrix $\operatorname{diag}(1,-1,-1)$ if characteristic $p \neq 2$ as well as of the matrix $\operatorname{diag}\left(J_{1}, J_{2}\right)$ for $p=2$.

The multiplicity vector of $A$ is often denoted by $m^{A}$. Given a string of matrices $A_{1}, \ldots, A_{k}$ we call $\left[m^{A_{1}}, \ldots, m^{A_{k}}\right]$ the multiplicity vector of $\left\{A_{1}, \ldots, A_{k}\right\}$. For practical purposes we need to distinguish the parts related to these $A_{i}$. To make it easier we usually express the above multiplicity vector as $\left[m^{A_{1}}\right] \ldots\left[m^{A_{k}}\right]$.

If $u=\left(a_{0}, \ldots, a_{l}\right)$ and $v=\left(b_{0}, \ldots, b_{l}\right)$ are two vectors then the standard inner product $\langle u, v\rangle$ is defined to be $a_{0} b_{0}+\cdots+a_{l} b_{l}$. We use this for multiplicity vectors. 


\section{Some facts on representations}

Some results in this section (in particular, Scott's theorem, 2.1) do not require $F$ to be algebraically closed. However, we prefer to hold this assumption as this is sufficient for our exposition.

We start by stating a theorem of $\operatorname{Scott}[\mathbf{1 9}]$. Let $G$ be any group and $V$ an $F G$ module. We set $d_{V}^{g}=\operatorname{dim} V^{g}$ and $d_{V}^{G}=\operatorname{dim} V^{G}$. Clearly, $V^{g}=V^{g^{-1}}$. For $g \in G$ set $\bar{d}_{V}^{g}=n-d_{V}^{g}$ and $\bar{d}_{V}^{G}=n-d_{V}^{G}$. Let $\hat{V}$ denote the dual module for $V$.

TheOREM 2.1. (Scott [19, Theorem 1]) Let $G$ be a group generated by elements $g_{1}, \ldots, g_{k}$ and set $g_{k+1}=g_{1} \cdots g_{k}$. Let $V$ be an $F G$-module. Then

$$
h_{V}\left(g_{1}, \ldots, g_{k}\right):=\bar{d}_{V}^{g_{1}}+\cdots+\bar{d}_{V}^{g_{k}}+\bar{d}_{V}^{g_{k+1}}-\bar{d}_{V}^{G}-\bar{d}_{\hat{V}}^{G} \geqslant 0 .
$$

REMARK. This result is stated in [19] with $g_{k+1}^{-1}$ in place of $g_{k+1}$. However, this is immaterial as $V^{g}=V^{g^{-1}}$.

It is convenient to us to deal with $d_{V}^{g}$ in place of $\bar{d}_{V}^{g}$. As $\bar{d}_{V}^{g}=n-d_{V}^{g}$ and $\bar{d}_{V}^{G}=n-d_{V}^{G}$, one can express (1) as

$$
(k-1) n+d_{V}^{G}+d_{\hat{V}}^{G}-d_{V}^{g_{1}}-\cdots-d_{V}^{g_{k}}-d_{V}^{g_{k+1}} \geqslant 0 .
$$

We set $d f_{V}^{G}=:(k-1) n-d_{V}^{g_{1}}-\cdots-d_{V}^{g_{k}}-d_{V}^{g_{k+1}}$ and we call $d f_{V}^{G}$ the defect of $G$ on $V$. The following lemma is obvious.

LEMMA 2.2. (1) $d f_{V}^{G} \geqslant-d_{V}^{G}-d_{\hat{V}}^{G}$.

(2) If $V=V_{1} \oplus V_{2}$ is a direct sum of FG-modules $V_{1}, V_{2}$ then $d f_{V}^{G}=d f_{V_{1}}^{G}+d f_{V_{2}}^{G}$.

(3) Let $V_{1}$ be a submodule of $V$ and $V_{2}=V / V_{1}$. Then $d f_{V}^{G} \geqslant d f_{V_{1}}^{G}+d f_{V_{2}}^{G}$. If $V$ is the sum of the $g_{i}$-eigenspaces for every $i=1, \ldots, k+1$ then $d f_{V}^{G}=d f_{V_{1}}^{G}+d f_{V_{2}}^{G}$.

If $k=2,(2)$ simplifies to

$$
d_{V}^{g_{1}}+d_{V}^{g_{2}}+d_{V}^{g_{3}} \leqslant n+d_{V}^{G}+d_{\hat{V}}^{G} .
$$

If $V$ is a non-trivial irreducible $G$-module (or more generally, $V^{G}=0$ and $\hat{V}^{G}=0$ ) then (2) takes shape

$$
d_{V}^{g_{1}}+\cdots+d_{V}^{g_{k}}+d_{V}^{g_{k+1}} \leqslant(k-1) n
$$

and (3) simplifies to

$$
d_{V}^{g_{1}}+d_{V}^{g_{2}}+d_{V}^{g_{3}} \leqslant n
$$

Formula (5) is very useful for deciding whether a particular group $G$ is Hurwitz. In practice, one starts with a $G$-module $V$ of minimal dimension greater than 1 . The efficiency of formula (5) is revealed in full when it applies to several $G$-modules. For $G$ being $\operatorname{SL}(n, q)$ or $\mathrm{SU}(n, q)$ the only useful modules turn out to be the natural one, its symmetric square (the exterior square if $p=2$ ) and the adjoint module. Another practical way of using Scott's formula is in applying it to tensor products. In this case, we have to use the language of representation theory. In the remaining part of this section we develop some machinery for doing this efficiently.

Let $V, W$ be $F G$-modules. We set $M=\operatorname{Hom}_{F}(V, W)$. Recall that the $G$-action which turns $M$ into an $F G$-module is defined as follows. Let $g \in G, f \in M$ and 
$v \in V$. Define $g \circ f \in \operatorname{Hom}_{F}(V, W)$ by $(g \circ f)(v)=g f\left(g^{-1}(v)\right)$. Clearly, $g \circ f$ is a linear mapping. In addition, for $g, h \in G$ we have $\left.(g h \circ f)(v)=g h f\left(h^{-1} g^{-1}\right) v\right)=$ $g\left((h \circ f)\left(g^{-1} v\right)\right)=(g \circ(h \circ f))(v)$.

The following fact is well known. We provide a proof for readers' convenience.

Lemma 2.3. Let $V, W$ be $F G$-modules. Then

$$
\operatorname{dim} \operatorname{Hom}_{F G}\left(1_{G}, \operatorname{Hom}_{F}(V, W)\right)=\operatorname{dim}_{\operatorname{Hom}_{F G}}(V, W) .
$$

Proof. Let $f \in \operatorname{Hom}_{F}(V, W)$ and $g \in G$. The mapping sending $g \in G$ to the linear transformation $f \rightarrow g \circ f$ is a representation of $G$. Next, $g \circ f=f$ means that $g f\left(g^{-1}(v)\right)=f(v)$ for all $v \in V$, so $f\left(g^{-1}(v)\right)=g^{-1}(f(v))$ for all $v \in V$. If this is true for all $g \in G$, then $f$ is a $F G$-module homomorphism. It follows that the subspace $X$ of $G$-fixed points on $\operatorname{Hom}_{F}(V, W)$ is isomorphic (as a vector space) to $\operatorname{Hom}_{F G}(V, W)$. Clearly, the dimension of $X$ is equal to that of $\operatorname{Hom}_{F G}\left(1_{G}, X\right)$ and we are done.

Corollary 2.4. If $V, W$ are some $F G$-modules then $\operatorname{Hom}_{F}(V, W)$ has no $G$-fixed point if and only if no quotient module of $V$ is isomorphic to a non-zero submodule of $W$. In particular, this happens if $V$ and $W$ are irreducible and $V$ is not isomorphic to $W$.

Now we state the following special case of Theorem 2.1 which is particularly useful for what follows.

Proposition 2.5. Let $G=\left\langle g_{1}, \ldots, g_{k}\right\rangle$ and $g_{k+1}=g_{1} \cdots g_{k}$. Let $V, W$ be some $F G$-modules and $M=\operatorname{Hom}_{F}(V, W)$. Set $d_{M}^{g_{k}}=\operatorname{dim} M^{g_{i}}$ for $i=1, \ldots, k+1$. Then

$$
\begin{aligned}
d_{M}^{g_{1}}+\cdots+d_{M}^{g_{k}}+d_{M}^{g_{k+1}} \leqslant & (k-1)(\operatorname{dim} V)(\operatorname{dim} W) \\
& +\operatorname{dim} \operatorname{Hom}_{F G}(V, W)+\operatorname{dim}_{\operatorname{Hom}_{F G}(W, V) .}
\end{aligned}
$$

If $V$ and $W$ are irreducible and $V$ is not isomorphic to $W$ then the right-hand side is $(k-1)(\operatorname{dim} V)(\operatorname{dim} W)$.

Proof. The first claim follows from Theorem 2.1 and the second one follows from Corollary 2.4.

The case where $M=\operatorname{Hom}(V, V)=$ End $V$ is of particular interest. Let $\lambda: G \rightarrow$ $\mathrm{GL}(V)$ be the representation associated with $V$. As End $V$ can be identified with the vector space $M(n, F)$ of all $(n \times n)$-matrices over $F$, the $G$-action defined above can be expressed as $g \circ x=g x g^{-1}$ for $g \in G, x \in M(n, F)$. Then $d_{M}^{g}$ is exactly the dimension of the vector space $C(g)$ of matrices commuting with $\lambda(G)$. We set $c_{V}^{g}:=d_{\operatorname{End} V}^{g}$ and similarly denote by $c_{V}^{G}$ the dimension of the vector space of matrices commuting elementwise with $\lambda(G)$. It follows from (6) that

$$
c_{V}^{g_{1}}+\cdots+c_{V}^{g_{k}}+c_{V}^{g_{k+1}} \leqslant(k-1) n^{2}+2 c_{V}^{G} .
$$

LEMma 2.6. If $V$ is irreducible then

$$
c_{V}^{g_{1}}+\cdots+c_{V}^{g_{k}}+c_{V}^{g_{k+1}} \leqslant(k-1) n^{2}+2 .
$$

Proof. By Schur's lemma, $c_{V}^{G}=1=c_{\hat{V}}^{G}$ so the result follows from the above. 
For $k=2,(8)$ simplifies to

$$
c_{V}^{g_{1}}+c_{V}^{g_{2}}+c_{V}^{g_{3}} \leqslant n^{2}+2 .
$$

We illustrate the use of the Scott's theorem by providing a new proof of the following classical fact.

Lemma 2.7. Let $G=\langle A, B\rangle \subset \mathrm{GL}(n, F)$ be an irreducible subgroup. Suppose that the minimum polynomials of $A, B$ are of degree 2 . Then $n=2$.

Proof. The multiplicity vectors for $A$ and $B$ are of shape $[a, n-a],[b, n-b]$ for some integers $a, b>0$, respectively. So $c_{V}^{A}=a^{2}+(n-a)^{2} \geqslant n^{2} / 2$ and $c_{V}^{B} \geqslant n^{2} / 2$. Hence $c_{V}^{A}+c_{V}^{B} \geqslant n^{2}$. By formula (9) $c_{V}^{A B} \leqslant 2$. This implies $n \leqslant 2$ as $c_{V}^{g} \geqslant n$ for any $(n \times n)$-matrix $g$.

Definition 2.8. Let $G=\left\langle g_{1}, \ldots, g_{k}\right\rangle$ and set $g_{k+1}=g_{1} \cdots g_{k}$. Let $L$ be a field and $\lambda: G \rightarrow \mathrm{GL}(n, F)$ an absolutely irreducible representation. The value

$$
\operatorname{ri}(\lambda)=:(k-1) n^{2}+2-c_{V}^{\lambda\left(g_{1}\right)}-\cdots-c_{V}^{\lambda\left(g_{k}\right)}-c_{V}^{\lambda\left(g_{k+1}\right)}
$$

is called the rigidity index of $\lambda$. If $\operatorname{ri}(\lambda)=0$, one says that $\lambda$ is rigid.

So a representation $\lambda$ is rigid if it is irreducible and $\operatorname{ri}(\lambda)=0$.

The following result (which motivates the term 'rigid') goes back to P. Deligne (see Simpson [17]).

THEOREM 2.9. Let $G=\left\langle g_{1}, \ldots, g_{k}\right\rangle$ and $g_{k+1}=g_{1} \cdots g_{k}$. Let $\lambda, \mu: G \rightarrow \operatorname{GL}(n, F)$ be representations such that matrix $\lambda\left(g_{i}\right)$ is similar to $\mu\left(g_{i}\right)$ for every $i=1, \ldots, k+1$.

(1) Suppose that $\lambda$ is rigid. Then $\mu$ is equivalent to $\lambda$. (Equivalently, if $\lambda$ and $\mu$ are non-equivalent then $\lambda$ is not rigid.)

(2) Suppose that $\lambda$ is not rigid. Then there exists a representation $\nu: G \rightarrow$ $\mathrm{GL}(n, F)$ not equivalent to $\lambda$ such that matrices $\lambda\left(g_{i}\right)$ and $\nu\left(g_{i}\right)$ are similar for every $i=1, \ldots, k+1$.

Proof (see [21]). We reproduce the proof of (1) here as this emphasizes the role played by Theorem 2.1. Suppose the contrary. Let $V, W$ be the $F G$-modules associated with $\lambda, \mu$, respectively. As $V$ and $W$ are not isomorphic and $V$ is irreducible, the right-hand side in (6) is $(k-1) n^{2}$. As $\lambda\left(g_{i}\right)$ is similar to $\mu\left(g_{i}\right)$ for $i=1, \ldots, k+1$, the left hand sides in (6) and in (7) coincide. In addition, they are equal to $(k-1) n^{2}+2$ as $\lambda$ is rigid. This is a contradiction.

The following useful result is an immediate consequence of Theorem 2.9.

Theorem 2.10. Let $G=\left\langle g_{1}, \ldots, g_{k}\right\rangle$ and $g_{k+1}=g_{1} \cdots g_{k}$. Let $L$ be a field and let $\lambda: G \rightarrow \mathrm{GL}(n, L)$ be a rigid representation. Let $\alpha$ be an automorphism of $L$ extended to $\mathrm{GL}(n, L)$ by the standard way. If a preserves the similarity classes of $\lambda\left(g_{1}\right), \ldots, \lambda\left(g_{k+1}\right)$ then $\lambda$ is equivalent to $\alpha \lambda$.

This can be used as follows.

TheOREM 2.11. Let $G=\left\langle g_{1}, \ldots, g_{k}\right\rangle$ and $g_{k+1}=g_{1} \cdots g_{k}$. Let $L$ be a finite field of characteristic $p$ and let $\lambda: G \rightarrow \operatorname{GL}(n, L)$ be an irreducible rigid representation. Let $K$ be a proper subfield of $L$ such that every $\lambda\left(g_{i}\right)$ for $i=1, \ldots, k+1$ is similar to a matrix in $\mathrm{GL}(n, K)$. Then $\lambda$ is equivalent to a representation into $\mathrm{GL}(n, K)$. 
Proof. Let $\alpha$ be a generator of the Galois group of $L / K$. Observe that $\alpha$ preserves the similarity class of $\lambda\left(g_{i}\right)$ for every $i=1, \ldots, k+1$. Indeed, $\lambda\left(g_{i}\right)$ is similar to a matrix over $K$ so $\alpha$ fixes a GL $(n, L)$-conjugate of $\lambda\left(g_{i}\right)$ hence the similarity class. By Theorem 2.10, $\lambda \cong \alpha \lambda$, that is, $\alpha(\lambda(g))=M \lambda(g) M^{-1}$ for some $M \in \mathrm{GL}(n, L)$ and $g \in G$. It follows that the trace $t(g)$ of $\lambda(g)$ is fixed by $\alpha$ for all $g \in G$. By the Galois theory, $t(g) \in K$ for all $g \in G$. As $\lambda$ is absolutely irreducible (by the definition of rigidity), the enveloping algebra $\langle\lambda(G)\rangle$ of $G$ over $K$ is simple and finite, hence is isomorphic to a matrix algebra $M(r, P)$ for some field $P$ which contains $K$ (Wedderburn's theorem). Here $r=n$ as $\lambda(G)$ contains $n^{2}$ linear independent matrices over $L$ by Burnside's theorem. As all traces $t(g)$ belong to $K$, we have that $K=P$.

The following theorem is rather well known.

TheOREM 2.12. Let $L$ and $\lambda$ be as in Theorem 2.11.

(1) Suppose that $L$ has an automorphism of order 2 and let $\sigma$ be the automorphism of $\mathrm{GL}(n, L)$ extending it. Suppose that every $\lambda\left(g_{i}\right)$ for $i=1, \ldots, k+1$ is similar to $\sigma\left(\lambda\left(g_{i}^{-1}\right)\right)$. Then $\lambda$ is equivalent to a representation into the unitary group $U(n, L)$.

(2) Suppose that $\lambda\left(g_{i}\right)$ is similar to $\lambda\left(g_{i}^{-1}\right)$ for every $i=1, \ldots, k+1$, that is, every $\lambda\left(g_{i}\right)$ is real. Then $\lambda$ is equivalent to a representation into either $\operatorname{Sp}(n, L)$ or $O(n, L)$.

Proof. For uniformity of the argument we declare $\sigma$ to be the trivial automorphism of $\mathrm{GL}(n, L)$ in case (2). For $x \in \mathrm{GL}(n, L)$ set $x^{*}=\sigma\left(\left(x^{-1}\right)\right)^{T}$ and $\lambda^{*}(g)=$ $\sigma\left(\lambda\left(g_{i}^{-1}\right)\right)^{T}$ for $g \in G$. Then $*$ is an involutory automorphism of GL $(n, L)$. Therefore, $g \rightarrow \lambda^{*}(g)$ is a representation of $G$ and $\lambda^{* *}=\lambda$. By assumption, the matrices $\lambda^{*}\left(g_{i}\right)$ and $\lambda\left(g_{i}\right)$ are similar for every $i=1, \ldots, k+1$ (as every matrix $x$ is similar to $\left.x^{T}\right)$. As $\lambda$ is rigid, $\lambda$ is equivalent to $\lambda^{*}$ by Theorem 2.9. So the result follows from [11, Lemma 2.10.15].

Set $R=M(n, F)$ and view $R$ as a $\mathrm{GL}(n, F)$-module under the congruence action (the congruence action is defined by sending each $M \in R$ to $g M g^{T}$ for $g \in \operatorname{GL}(n, F)$ where $g^{T}$ stands for the transpose of $\left.g\right)$. Let $V$ be the natural GL $(n, F)$-module. We denote by $S=S(V)$ the $\mathrm{GL}(n, F)$-module of all symmetric bilinear forms on $V$. It becomes a $\mathrm{GL}(n, F)$-module if one defines the action of $g \in \mathrm{GL}(n, F)$ on $f(x, y) \in S$ for $x, y \in V$ by $(g f)(x, y)=f\left(g^{T} x, g^{T} y\right)$. (Here we use the transpose of $g$ to have $S$ a left module.) Then $S$ can be identified with the set of all symmetric matrices viewed as a $\mathrm{GL}(n, F)$-module under the congruence action. Similarly, denote by $E=E(V)$ the $\mathrm{GL}(n, F)$-module of all alternating bilinear forms on $V$. If $p \neq 2$, then $E$ can be identified with the set of all skew symmetric matrices viewed as a $\mathrm{GL}(n, F)$-module under the congruence action. In addition, $R=S \oplus E$. If $p=2$ then $E$ can be identified with the set of all symmetric matrices with zero diagonal. It is a classical fact that $E$ is an irreducible $\operatorname{GL}(n, F)$-module and, if $p \neq 2$, so is $S$. If $p=2$ then $S / E$ is irreducible of dimension $n$ and $E$ is a unique minimal submodule of $S$ and of $R$. Recall that $\operatorname{dim} S=n(n+1) / 2$ and $\operatorname{dim} E=n(n-1) / 2$. Observe that $R=M(n, F)$ as a $\operatorname{GL}(n, F)$-module under the congruence action is isomorphic to $\operatorname{Hom}(V, \hat{V}) \cong V \otimes V$. Therefore, $S$ is isomorphic to the symmetric square of $V$ which is the subspace of $V \otimes V$ spanned by $v \otimes v$ and $v \otimes v^{\prime}+v^{\prime} \otimes v$ for $v, v^{\prime} \in V$. 
LEMma 2.13. Let $G \subset \mathrm{GL}(V)$ be a subgroup which preserves a non-degenerate bilinear form $f$ on $V$. Let $W$ be a $G$-stable subspace of $V$. Then $\hat{W} \cong V / W^{\perp}$ ( a $G$-module isomorphism).

Proof. For $u \in V$ define a mapping $\alpha_{u}: V \rightarrow F$ as $\alpha_{u}(v)=f(u, v)$. Then $\alpha_{u} \in \hat{V}$ and $u \rightarrow \alpha_{u}$ is a $G$-module isomorphism $V \rightarrow \hat{V}$. Observe that the restriction $\left.\alpha_{u}\right|_{W}$ of $\alpha_{u}$ to $W$ belongs to $\hat{W}$ and the kernel of the linear mapping $\left.u \rightarrow \alpha_{u}\right|_{W}$ is $W^{\perp}$. (The kernel consists of $u$ such that $\left.\alpha_{u}\right|_{W}=0$, equivalently, $f(u, W)=0$.) Hence $V / W^{\perp} \cong \hat{W}$ as required.

The following is well known.

Lemma 2.14. (1) The dual $\hat{E}$ of $E$ is isomorphic to $E(\hat{V})$.

(2) If $p \neq 2$ then the dual $\hat{S}$ of $S$ is isomorphic to $S(\hat{V})$.

(3) If $p=2$ then $\hat{S} \cong R / E$.

Proof. (1) Define a mapping $\alpha: R \rightarrow R$ by $\alpha(M)=M-M^{T}$ for $M \in R$. Then the image of $\alpha$ belongs to $E$ and the kernel of $\alpha$ coincides with $S$. (This is true for $p=2$ as well as for $p \neq 2$.) By a dimension reason, the image of $\alpha$ coincides with $E$. As $\alpha$ is a $\operatorname{GL}(n, F)$-module homomorphism, we have that $E \cong R / S$. As $E$ is irreducible, so is $\hat{E}$. It follows that $\hat{E}$ is a minimal submodule of the dual of $R$; that is, of $\operatorname{Hom}(\hat{V}, V) \cong \hat{V} \otimes \hat{V}$. However, $\hat{V} \otimes \hat{V}$ contains $E(\hat{V})$ as a minimal submodule. So the result follows.

(2) Similarly, define a mapping $\beta: R \rightarrow R$ by $\beta(M)=M+M^{T}$. Then the image of $\beta$ belongs to $S$. As $p \neq 2$, the kernel of $\beta$ coincides with $E$. Now a similar argument yields the result.

(3) Define a non-degenerate bilinear form on $R$ by $f(A, B)=\operatorname{Tr}(A B)$ for $A, B \in$ $R$ where $\operatorname{Tr}$ stands for the trace of a matrix. The result follows by taking $R$ for $V$ and $S$ for $W$ in Lemma 2.13. Indeed, $S=\left\{Y \in R: Y=Y^{T}\right\}$ and $E=\left\{X+X^{T}: X \in\right.$ $R\}$. Hence $f\left(X+X^{T}, Y\right)=\operatorname{Tr}(X Y)+\operatorname{Tr}\left(X^{T} Y\right)=0$ as $\operatorname{Tr}\left(X^{T} Y\right)=\operatorname{Tr}\left(Y X^{T}\right)=$ $\operatorname{Tr}(X Y)^{T}$. So $f(E, S)=0$. As $\operatorname{dim} S+\operatorname{dim} E=\operatorname{dim} R$, the result follows.

REMARK. If $p=2$ and $n>3$ then $\hat{S}$ is not isomorphic to $S(\hat{V})$. Indeed, in this case $\hat{S}$ has an irreducible quotient module isomorphic to $\hat{E}$ so it has a submodule of dimension $n$ in contrast with $S(\hat{V})$ which has a unique irreducible submodule of dimension $n(n-1) / 2$.

Lemma 2.15. Let $G \subset \mathrm{GL}(n, F)$ be an irreducible subgroup. Then $\operatorname{dim} S^{G} \leqslant 1$ and $\operatorname{dim} E^{G} \leqslant 1$.

Proof. For $i=1,2$ let $\Gamma_{i} \in S$ and $g \Gamma_{i} g^{T}=\Gamma_{i}$ for all $g \in G$. As $G$ is irreducible, each $\Gamma_{i}$ is non-degenerate so $\Gamma_{1}^{-1} g \Gamma_{1}=\left(g^{T}\right)^{-1}=\Gamma_{2}^{-1} g \Gamma_{2}$. Hence $\Gamma_{2} \Gamma_{1}^{-1}$ centralizes $G$. By Schur's lemma, $\Gamma_{2} \Gamma_{1}^{-1}$ is scalar, whence the result. The second claim follows by the same argument for $\Gamma_{i} \in E$.

Let $K$ denote the $F G$-module of all quadratic forms on $V$.

Lemma 2.16. Let $n, q$ be even. Then $K \cong R / E \cong \hat{S}$. 
Proof. Denote by $T$ the subspace of upper triangular matrices in $R$. Then $T \cap E=0$ and $\operatorname{dim} T+\operatorname{dim} E=n^{2}$. Hence $T \oplus E=R$. A quadratic form $Q: F^{n} \rightarrow F$ is defined for $\left(x_{1}, \ldots, x_{n}\right)^{T} \in F^{n}$ by the formula:

$$
\sum_{i} \alpha_{i i} x_{i}^{2}+\sum_{k<l} \alpha_{k l} x_{k} x_{l}
$$

where $\alpha_{i j} \in F$. Denote by $t(Q)$ the upper triangular matrix whose the $(k, l)$-entries for $k \leqslant l$ are $\alpha_{k l}$. We call $t(Q)$ the matrix of $Q$. Thus, $Q \rightarrow t(Q)$ is a bijection $t: K \rightarrow T$. Moreover, $t$ is a vector space isomorphism. For $g \in \mathrm{GL}(n, q)$ the action of $g$ on $K$ is defined by $(g Q)(v)=Q\left(g^{T} v\right)$ for $v=\left(x_{1}, \ldots, x_{n}\right)^{T} \in F^{n}$. Under this action $K$ becomes an GL $(n, F)$-module. To show that the $\mathrm{GL}(n, F)$-modules $K$ and $R / E$ are isomorphic, we have to prove that $t\left((g(Q))+g t(Q) g^{T} \in E\right.$. It suffices to prove this for $Q=x_{k} x_{l}$ with $k \leqslant l$ as the mapping $Q \rightarrow t(g(Q))+g t(Q) g^{T}$ is linear. Therefore, in this case $g(Q) v=\left(g^{T} v\right)_{k}\left(g^{T} v\right)_{l}=\left(\sum_{i} g_{i k} x_{i}\right)\left(\sum_{j} g_{j l} x_{j}\right)=$ $\sum_{i j} g_{i k} g_{j l} x_{i} x_{j}$. Therefore, the $(i, j)$-entry of the matrix $t(g(Q))$ is $g_{i k} g_{j l}+g_{j k} g_{i l}$ for $i<j$ and $g_{i k} g_{i l}$ for $i=j$. On the other hand, $t(Q)=E_{k l}$, the matrix with $(k, l)$ entry equal to 1 and 0 elsewhere. The $(i, j)$-entry of $g E_{k l} g^{T}$ is $g_{i k} g_{j l}$. Therefore, the diagonal entries of $t(g(Q))$ and $g^{T} t(Q) g \in E$ are the same, while the off-diagonal entries differ by matrices from $E$. This proves that $K \cong R / E$ and $R / E \cong \hat{S}$ by Lemma 2.14(3).

Lemma 2.17. Let $p=2$ and let $G$ be an irreducible subgroup of $\operatorname{GL}(n, q)$ where $n, q$ are even.

(1) $\operatorname{dim} K^{G} \leqslant 1$.

(2) If $G$ preserves a symmetric bilinear form and preserves no quadratic form then $\operatorname{dim} K^{G}=0$ and $\operatorname{dim} \hat{K}^{G}=1$ (equivalently, $\operatorname{dim} \hat{S}^{G}=0$ and $\operatorname{dim} S^{G}=1$ ).

Proof. (1) Observe that $K$ contains a submodule $L$ formed by the squares of the linear forms on $V$. Clearly, $\operatorname{dim} L=n$ and $L$ is irreducible. So $\operatorname{dim} K / L=n(n-1) / 2$. The kernel of the polarization mapping $\pi$ (which sends every $Q \in K$ to the bilinear form $Q(u+v)+Q(u)+Q(v)$ for $u, v \in V)$ ) coincides with $L$. Therefore, $K / L \cong E$. As $L$ is irreducible, $L \cap K^{G}=0$. So $\operatorname{dim} K^{G}=\operatorname{dim} \pi\left(K^{G}\right)$. As $\pi\left(K^{G}\right) \subseteq E^{G} \subseteq S^{G}$ and $\operatorname{dim} S^{G} \leqslant 1$ by Lemma 2.15 , the result follows.

(2) It is obvious that $\operatorname{dim} K^{G}=0$. As $\hat{K} \cong S$, the assertion follows from Lemma 2.15 .

Proposition 2.18. Let $G=\left\langle g_{1}, \ldots, g_{k}\right\rangle \subset \mathrm{GL}(n, F)$ be an irreducible group and set $g_{k+1}=g_{1} \cdots g_{k}$. Let $V=F^{n}$ and let $S$ be the set of all symmetric matrices in $M(n, F)$ viewed as an $F G$-module under the congruence action. For $g \in G$ set $d_{S}^{g}=\operatorname{dim} S^{g}$ and $d f_{S}^{G}=(k-1) \operatorname{dim} S-d_{S}^{g_{1}}-\cdots-d_{S}^{g_{k+1}}$.

(1) $\operatorname{dim} E^{G}=\operatorname{dim} \hat{E}^{G} \leqslant 1$. If $p \neq 2$ then $\operatorname{dim} S^{G}=(k-1) \operatorname{dim} \hat{S}^{G} \leqslant 1$; if $p=2$ then $S^{G}=0$ implies $\hat{S}^{G}=0$.

(2) $d f_{E}^{G} \geqslant-2$ and $d f_{S}^{G} \geqslant-2$.

(3) If $G$ preserves no symmetric bilinear form then $d f_{S}^{G} \geqslant 0$; if $p=2$ then, additionally, $d f_{E}^{G} \geqslant 0$. If $p \neq 2$ and $G$ preserves no skew symmetric form then $d f_{E}^{G} \geqslant 0$.

(4) If $p=2$ and $G$ preserves a symmetric bilinear form but no quadratic form then $\operatorname{dim} \hat{S}^{G}=0$ and $d f_{S}^{G} \geqslant-1$. 
Proof. (1) As $G$ is irreducible on $V$, it is irreducible on $\hat{V}$. By Lemma 2.15, $\operatorname{dim} E^{G} \leqslant 1$ and $\operatorname{dim} S^{G} \leqslant 1$. By Lemma $2.14, \operatorname{dim} \hat{E}^{G} \leqslant 1$. If $\operatorname{dim} E^{G}$ or $\operatorname{dim} \hat{E}^{G}$ equals 1 then $G$ preserves a symplectic form on $V$; hence $V$ is a self-dual $G$-module. Then $\operatorname{dim} E^{G}=\operatorname{dim} \hat{E}^{G}=1$. Let $p \neq 2$. The equality $\operatorname{dim} S^{G}=1$ means that $G$ preserves a symmetric bilinear form $f$ on $V$. As $G$ is irreducible, $f$ is non-degenerate. Let $\Gamma$ be the matrix of $f$ relative to some basis so $\Gamma$ is non-degenerate and $g \Gamma g^{T}=\Gamma$ for all $g \in G$. Then $\Gamma^{-1} g \Gamma=\left(g^{T}\right)^{-1}$ which implies that $V$ is a selfdual $F G$-module, that is, $V \cong \hat{V}$. By Lemma $2.14, S \cong \hat{S}$ so $\operatorname{dim} \hat{S}^{G}=1$. The proof of the converse is similar. Let $p=2$. It is well known that $K^{G} \neq 0$ implies that $S^{G} \neq 0$ (see also the proof of Lemma 2.17(1)). As $\hat{S}=K$ by Lemma 2.16, the result follows.

(2) By formula (3) applied to $S$ we have that $d f_{S}^{G} \geqslant-\operatorname{dim} S^{G}-\operatorname{dim} \hat{S}^{G}$, and the right-hand side is not less than -2 by Lemma 2.15, Lemma 2.14 (for $p \neq 2$ ) and Lemma 2.17 (for $p=2$ ). Similarly, $-\operatorname{dim} E^{G}-\operatorname{dim} \hat{E}^{G} \geqslant-2$ by Lemmas 2.15 and 2.14 .

(3) By formula (3) applied to $S$ we have $d f_{S}^{G}+\operatorname{dim} S^{G}+\operatorname{dim} \hat{S}^{G} \geqslant 0$. As $S^{G}=0$, by (1) we have $\hat{S}^{G}=0$ and the result follows. If $p=2$ then $E \subset S$ so $S^{G}=0$ implies $E^{G}=0$. By Lemma 2.14, $\hat{E} \cong E(\hat{V})$ hence $\hat{E}^{G}=0$, and (3) applied to $E$ yields the result.

(4) As $\hat{S} \cong K$ (Lemma 2.16), we have $\hat{S}^{G}=K^{G}=0$ and $\operatorname{dim} S^{G}=1$. So the result follows from Lemma 2.2.

Verifying formulas for $S$ from items (2), (3), (4) of Proposition 2.18 for a given multiplicity vector is called the symmetric test, and verifying those for $E$ is called the exterior test. We refer to them as $T_{S}$ and $T_{E}$, respectively. To be precise, if $G$ is irreducible then we have:

$$
T_{S}= \begin{cases}d f_{S}^{G} \geqslant-2 & \\ d f_{S}^{G} \geqslant 0 & \text { if } G \text { preserves no non-degenerate symmetric bilinear form, } \\ d f_{S}^{G} \geqslant-1 & \text { if } p=2 \text { and } G \text { preserves a non-degenerate alternating } \\ & \text { bilinear form, and no quadratic form. }\end{cases}
$$

Similarly,

$$
T_{E}=\left\{\begin{array}{l}
d f_{E}^{G} \geqslant-2 \\
d f_{E}^{G} \geqslant 0
\end{array} \text { if } G\right. \text { preserves no skew symmetric bilinear form. }
$$

Additionally, verifying formula (8) is called the adjoint test which is refered to as $T_{A}$. Computational aspects of this matter for $k=2$ are discussed in Section 4 .

We shall regularly apply Theorem 2.1 to the $F G$-module $M=\operatorname{Hom}(V, W)$ where $V, W$ are irreducible $F G$-modules. Lemma 2.3 gives us the right-hand side values $d_{M}^{G}$ and $d_{\hat{M}}^{G}$ for formulas (2), (4) and (5) above, that is, with $M$ in place of $V$. Namely, $d_{M}^{G}=\operatorname{dim}_{H_{F G}}(V, W)$ and $d_{\hat{M}}^{G}=\operatorname{dim}_{H_{F o m}}(W, V)$, because $\hat{M} \cong \operatorname{Hom}(W, V)$. We next discuss some aspects of computing the left-hand side. Let $g \in G$ with $g^{l}=1$ and let $A, B$ be the matrices of the action of $g$ in $V, W$, respectively. Suppose first that $A, B$ are diagonalizable with multiplicity vectors $m^{A}=\left[m_{0}^{A}, m_{1}^{A}, \ldots, m_{l-1}^{A}\right]$ and $m^{B}=\left[m_{0}^{B}, m_{1}^{B}, \ldots, m_{l-1}^{B}\right]$, respectively. Then, by the definition of the action of $g$ on $M=\operatorname{Hom}_{F}(V, W)$, the multiplicity $d_{M}^{g}$ of eigenvalue 1 of the action of $g$ on $M$ is $\sum_{i=0}^{l-1} m_{i}^{A} m_{i}^{B}$. This can be viewed as the inner product of the multiplicity vectors if one regards them as elements of $\mathbf{Q}^{l}$. Therefore, if we denote by $m_{V}^{g}$ and $m_{W}^{g}$ the 
multiplicity vectors $m^{A}$ and $m^{B}$ of $g$ on $V, W$, respectively, then $d_{M}^{g}=\left\langle m_{V}^{g}, m_{W}^{g}\right\rangle$ where $\langle$,$\rangle is used to denote the inner product.$

Furthermore, we show that formula $d_{M}^{g}=\left\langle m_{V}^{g}, m_{W}^{g}\right\rangle$ holds when $A, B$ are unipotent matrices. Let $s_{1} \geqslant \cdots \geqslant s_{k}$ and $t_{1} \geqslant \cdots \geqslant t_{l}$ be the sizes of the blocks in the Jordan form of $A$ and $B$, respectively. Then $d_{M}^{g}=\operatorname{dim}_{F\langle g\rangle}(V, W)=$ $\sum_{i, j} \min \left\{s_{i}, t_{j}\right\}$; see, for instance, Humphreys [9, Section 1.2]. One can show that the right-hand side value is equal to the inner product $\left\langle m_{V}^{g}, m_{W}^{g}\right\rangle$. To do this, one can use diagrams $Y(A)$ for the partitions $\operatorname{dim} V=s_{1}+\cdots+s_{k}$. Young diagrams look like

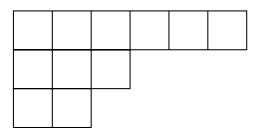

where the $i$ th row consists of $s_{i}$ boxes. One observes that the coordinates of the multiplicity vector $m^{A}$ are exactly the lengths of the columns of $Y(A)$. Next one can use induction on $k$ to establish the inner product formula.

If $V=W$ and $m_{V}^{g}=\left[m_{0}^{g}, \ldots, m_{l-1}^{g}\right]$ is the multiplicity vector for $g$ then $c_{V}^{g}=$ $d_{M}^{g}=\sum_{i}\left(m_{i}^{g}\right)^{2}$ which is trivial for semisimple matrices but not obvious in general (of course, this is well known; see for instance [9, Section 1.3]).

In fact, the inner product formula can be extended to arbitrary matrices. For this, we introduce a notion of a multiplicity function in place of a multiplicity vector. Let $\alpha \in F$ and $A \in$ End $V$. Set $m_{i}^{A}(\alpha)=\operatorname{dim}(\alpha \cdot \operatorname{Id}-A)^{i} V-\operatorname{dim}(\alpha \cdot \operatorname{Id}-A)^{i+1} V$ (here we set $\left.(\alpha \cdot \operatorname{Id}-A)^{0} V=V\right)$. Let $Z^{+}$denote the set of all non-negative integers. The function $m^{A}: F \rightarrow Z^{+} \times \cdots \times Z^{+}$defined by $\alpha \rightarrow\left[m_{0}(\alpha), m_{1}(\alpha), \ldots\right]$ is called the multiplicity function for $A$. This is a generalization of the notion of a multiplicity vector for unipotent matrices, and, in a sense, it also extends the notion of a multiplicity vector introduced for semisimple matrices of finite order. Clearly, $m^{A}(\alpha)=0$ if $\alpha$ is not an eigenvalue of $A$. If $A, B$ are square $F$-matrices (not necessarily of the same size), we set $\left\langle m^{A}, m^{B}\right\rangle=\sum_{\alpha \in F}\left\langle m^{A}(\alpha), m^{B}(\alpha)\right\rangle=$ $\sum_{\alpha \in F} \sum_{i} m_{i}^{A}(\alpha) m_{i}^{B}(\alpha)$. Obviously, this sum contains only finitely many non-zero terms. If $V$ is a $G$-module, and $A$ is the matrix of the action of $g \in G$ on $V$, we usually write $m^{g}$ for $m^{A}$, which will not lead to any confusion as it corresponds to the traditional convention.

The above argument for unipotent matrices works in the case where each $A$ and $B$ has only one eigenvalue, common for $A$ and $B$. As distinct eigenvalues do not actually interfere, we have the following lemma.

Lemma 2.19. Let $M=\operatorname{Hom}(V, W)$ and $g \in G$. Then $d_{M}^{g}=\left\langle m_{V}^{g}, m_{W}^{g}\right\rangle$.

To be more accurate, one can argue as follows. Set $V_{\alpha}=\{v \in V:(A-\alpha$. $\mathrm{Id})^{r} v=0$ for some $\left.r\right\}$. Then $\operatorname{Hom}_{F\langle g\rangle}(V, W)=\sum_{\alpha \in F} \operatorname{Hom}_{F\langle g\rangle}\left(V_{\alpha}, W_{\alpha}\right)$ so $d_{M}^{g}=$ $\operatorname{dim} \operatorname{Hom}_{F\langle g\rangle}(V, W)=\sum_{\alpha \in F} \operatorname{dim} \operatorname{Hom}_{F\langle g\rangle}\left(V_{\alpha}, W_{\alpha}\right)=\sum_{\alpha}\left\langle m_{V}^{g}(\alpha), m_{W}^{g}(\alpha)\right\rangle$.

If $V=W$ then $c_{V}^{g}=\sum_{\alpha \in F} \sum_{i}\left(m_{i}(\alpha)\right)^{2}$ as $c_{V}^{g}=d_{M}^{g}$ in this case. This yields the following.

Lemma 2.20. Let $\lambda: G \rightarrow \mathrm{GL}(n, F)$ be an irreducible representation. Then the rigidity index is even. 
Proof. By Definition 2.8, ri $(\lambda)=-2 n^{2}+2+\sum_{i=1}^{k+1}\left(n^{2}-c_{V}^{\lambda\left(g_{i}\right)}\right)$ so it suffices to show that $n^{2}-c_{V}^{\lambda(g)}$ is even for any $g \in G$. Let $\left[m_{1}, \ldots, m_{l}\right]$ be the multiplicity function of $g$. Then $m_{1}+\cdots+m_{l}=n$ and $c_{V}^{\lambda(g)}=m_{1}^{2}+\cdots+m_{l}^{2}=n^{2}-2 \sum_{i<j} m_{i} m_{j}$.

The following well known fact is used many times without a reference, especially in the cases where $g$ is semisimple.

LEMma 2.21. Let $g \in \mathrm{GL}(n, F)$ and let $k$ be the degree of the minimum polynomial of $g$. Then $c^{g} \leqslant n^{2} / k$.

Proof. Let $m^{g}(\alpha)=\left[m_{0}(\alpha), \ldots\right]$ be the multiplicity function of $g$. Then $c^{g}=$ $\sum_{\alpha \in F} \sum_{i}\left(m_{i}(\alpha)\right)^{2}$. Observe that $k$ is equal to the number of non-zero terms in this sum. (Indeed, the minimum polynomial of $g$ makes shape $\Pi_{\alpha}(x-\alpha)^{k_{\alpha}}$ where $k_{\alpha}$ is the maximum size of a Jordan block of $g$ with eigenvalue $\alpha$. In addition, $m_{i}(\alpha)=\operatorname{dim}(\alpha \cdot \operatorname{Id}-g)^{i} V-\operatorname{dim}(\alpha \cdot \mathrm{Id}-g)^{i+1} V$ which is non-zero if and only if $i+1 \leqslant k_{\alpha}$. So the claim follows.) Recall that $\sum_{\alpha \in F} \sum_{i} m_{i}(\alpha)=n$. So the lemma follows from the well known inequalities $\sum_{i=1}^{k} x_{i}^{2} \geqslant\left(x_{1}+\cdots+x_{k}\right)^{2} / k$ for real numbers $x_{1}, \ldots, x_{k}$.

The notion of a multiplicity vector (function) can be extended to strings of matrices. Say, if $A_{1}, \ldots, A_{k}$ is a string of $(n \times n)$-matrices over $F$, we define the multiplicity vector (function) $m^{A_{1}, \ldots, A_{k}}$ for the string to be $\left[m^{A_{1}}, \ldots, m^{A_{k}}\right]$. If $B_{1}, \ldots, B_{k}$ is a string of $(l \times l)$-matrices over $F$ with the same $k$, then we set $\left\langle m^{A_{1}, \ldots, A_{k}}, m^{B_{1}, \ldots, B_{k}}\right\rangle=\sum_{i=1}^{k}\left\langle m^{A_{i}}, m^{B_{i}}\right\rangle$. This can be used when $V, W$ are $G$ modules and $A_{i}, B_{i}$ are the matrices of the action of $g_{i} \in G$ on $V, W$, respectively. We also write $m_{V}^{g_{i}}$ for $m_{V}^{A_{i}}$ in this and similar situations.

If $V, W$ are irreducible and non-isomorphic then formula (6) can be expressed as

$$
\left\langle m_{V}^{g_{1}, \ldots, g_{k+1}}, m_{W}^{g_{1}, \ldots, g_{k+1}}\right\rangle=\sum_{i=1}^{k+1}\left\langle m_{V}^{g_{i}}, m_{W}^{g_{i}}\right\rangle \leqslant(k-1)(\operatorname{dim} V)(\operatorname{dim} W),
$$

and we often omit the superscript $g_{1}, \ldots, g_{k+1}$. If $V \cong W$ then

$$
\sum_{i=1}^{k+1} c_{V}^{g_{i}}=\sum_{i=1}^{k+1}\left\langle m_{V}^{g_{i}}, m_{V}^{g_{i}}\right\rangle \leqslant(k-1)(\operatorname{dim} V)^{2}+2 .
$$

Verifying formula (10) for some actual irreducible $F G$-module $W$ and a virtual irreducible $F G$-module $V$ with given multiplicity vector is called the tensor test. This complements the adjoint test, symmetric test and exterior test for $V$ discussed above. They are used as conditions of the non-existence of $V$ when we argue by the way of contradiction. If formula (10) holds, we say that $V$ passes the tensor test with $W$, otherwise we say that $V$ fails the tensor test with $W$ which means that we have a contradiction, and hence $V$ does not exist. If $V, W$ are not assumed to be non-isomorphic then failing formula (10) means that $V, W$ must be isomorphic. One observes that this is exactly the argument establishing Theorem 2.9(1). Of course if $V$ is an actual $F G$-module, it passes every test. As tests examine only the multiplicity vector $\left[m_{V}^{g_{1}}, \ldots, m_{V}^{g_{k+1}}\right]$, failing the test means that there is no irreducible $F G$-module $V$ with this multiplicity vector. 
ExAmPle. Let $G=H_{237}$. Lemmas $3.7(p \neq 2,7)$ and $3.8(p \neq 2,3)$ produce irreducible $F G$-modules $W$ with multiplicity vector $[4,2][2,2,2][0,1,1,1,1,1,1]$ and $[3,4][1,3,3][1,1,1,1,1,1,1]$, respectively. It is easy to check that formula (10) yields that $2 d_{V}^{x} \leqslant \operatorname{dim} V+d_{V}^{x y}$ and $\operatorname{dim} V \leqslant d_{V}^{x}+2 d_{V}^{y}$. These complement Scott's formula (5) which says that $d_{V}^{x}+d_{V}^{y}+d_{V}^{x y} \leqslant \operatorname{dim} V$.

Section 3 provides further examples of $\mathrm{FH}_{237}$-modules, and the tables given in Appendix B record more formulas, similar to those in the above example.

A multiplicity vector (function) is called basic if it is not the sum of the multiplicity vectors (functions) of actual representations of $G$. A representation (and corresponding module) is called basic if its multiplicity vector (function) is basic.

Let $U, V, W$ be irreducible $F G$-modules with multiplicity vectors (functions) $m_{U}, m_{V}, m_{W}$, respectively. Suppose that $m_{U}=m_{V}+m_{W}$. Obviously, if some module $N$ passes the tensor tests with $V, W$ then it passes the test with $U$. Therefore, such $U$ is not useful for testing. Thus, the only representations useful for tensor tests are basic. Obviously, if $\phi=\rho \otimes \tau$ is basic then $\rho$ and $\tau$ are basic. There is a connection between basic and rigid representations:

LEMMA 2.22. (1) Every rigid representation is basic.

(2) If a multiplicity vector is not basic, it has to pass every tensor test.

Proof. (1) Suppose that $R$ is a rigid $F G$-module. By the way of contradiction, let $M_{1}, \ldots, M_{t}$ be non-zero irreducible $F G$-modules such that $m_{R}=\sum m_{M_{i}}$. Then $\left\langle m_{M_{i}}, m_{R}\right\rangle \leqslant(k-1)\left(\operatorname{dim} M_{i}\right)(\operatorname{dim} R)$ by formula $(10)$. Hence $\left\langle m_{R}, m_{R}\right\rangle \leqslant$ $(k-1)(\operatorname{dim} R)(\operatorname{dim} R)$ while the rigidity requires $\left\langle m_{R}, m_{R}\right\rangle=(k-1)(\operatorname{dim} R)^{2}+2$.

(2) This is obvious.

The tensor product of two representations $\rho, \tau$ can be a rigid representation; see Lemmas 3.12, 3.14 and 3.15 below. We show in Theorem 2.26 that if $\rho \otimes \tau$ is rigid then both $\rho, \tau$ are rigid. For this we need a few preparatory observations.

Let $C=\langle g\rangle$ be a cyclic group, let $\rho: C \rightarrow \mathrm{GL}(n, F)$ be a representation and set $m^{g}=m^{\rho(g)}$ for the multiplicity function of $\rho(g)$. Let $V$ be the natural module for $\operatorname{GL}(n, F)$ and set $H=\operatorname{Hom}_{F}(V, V)$. If $\alpha$ is an eigenvalue of $g$ on $H$, we set $H_{\alpha}=\left\{x \in H:(g-\alpha \cdot \mathrm{Id})^{k} x=0\right.$ for some $\left.k=k(x)\right\}$.

We denote by $\mu^{g}$ the multiplicity function of $g$ on $\operatorname{Hom}(V, V)$. Thus, $\mu^{g}(\alpha)=$ $\left[\mu_{0}^{g}(\alpha), \mu_{1}^{g}(\alpha), \ldots\right]$ for $\alpha \in F$. Hence $\mu_{0}^{g}(1)=c^{g}$ is the dimension of the 1-eigenspace of $g$ on $\operatorname{Hom}(V, V)$.

Lemma 2.23. Let $\alpha \in F$. Then $\mu_{i}^{g}(\alpha) \leqslant \mu_{0}^{g}(\alpha) \leqslant \mu_{0}^{g}(1)=c^{g}$.

Proof. The first inequality is well known. Express $\rho(g)=D U$ where $D$ is diagonalizable and $U$ is a unipotent matrix such that $D U=U D$. Then $H_{\alpha}$ is exactly the $\alpha$-eigenspace of $D$ on $H$. Hence $H_{\alpha}=\oplus_{\beta \in F} \operatorname{Hom}\left(V_{\beta}, V_{\alpha \beta}\right)$ is an $F C$ module isomorphism (the sum is finite as $V_{\beta}=0$ if $\beta$ is not an eigenvalue of $D)$. Let $m(\beta)=\left[m_{0}(\beta), m_{1}(\beta), \ldots\right]$ denote the multiplicity vector of $\left.U\right|_{V_{\beta}}$. By Lemma 2.19, the dimension of the 1-eigenspace of $U$ on $\operatorname{Hom}\left(V_{\beta}, V_{\alpha \beta}\right)$ is equal to $\langle m(\beta), m(\alpha \beta)\rangle$ so $\mu_{0}^{g}(\alpha)$, the dimension of the 1-eigenspace of $U$ on $H_{\alpha}$, is equal to 
$\sum_{j} m_{j}(\beta) m_{j}(\alpha \beta)$. Therefore, $\mu_{0}^{g}(1)$, the dimension of the 1-eigenspace of $g$ on $H$, is equal to $\sum_{\beta} \sum_{j} m_{j}(\beta) m_{j}(\alpha \beta)$. If $\alpha=1$ then this is equal to $\sum_{\beta} \sum_{j}\left(m_{j}(\beta)\right)^{2}$. So

$$
\begin{aligned}
\mu_{0}^{g}(1)-\mu_{0}^{g}(\alpha) & =\sum_{\beta} \sum_{j}\left(m_{j}(\beta)\right)^{2}-\sum_{\beta} \sum_{j} m_{j}(\beta) m_{j}(\alpha \beta) \\
& =\frac{1}{2} \sum_{\beta} \sum_{j}\left(m_{j}(\beta)-m_{j}(\alpha \beta)\right)^{2}
\end{aligned}
$$

as $\sum_{\beta} \sum_{j}\left(m_{j}(\beta)\right)^{2}=\sum_{\beta} \sum_{j}\left(m_{j}(\alpha \beta)\right)^{2}$.

Let $\tau$ be another irreducible representation of $C$ realized in a module $W$.

LEMMA 2.24. Let $V, W$ be $F C$-modules.

(1) FC-modules $\operatorname{Hom}_{F}(V \otimes W, V \otimes W)$ and $\left.\operatorname{Hom}_{F}(V \otimes V) \otimes \operatorname{Hom}_{F}(W, W)\right)$ are isomorphic.

(2) Let $\mu^{g}, \mu^{\tau(g)}, \mu^{(\rho \otimes \tau)(g)}$ denote the multiplicity functions of $g$ on $\operatorname{Hom}(V, V)$, $\operatorname{Hom}(W, W)$ and $\operatorname{Hom}(V \otimes W, V \otimes W)$, respectively. Then

$$
\mu_{0}^{(\rho \otimes \tau)(g)}(1)=\sum_{\alpha}\left\langle\mu^{g}(\alpha), \mu^{\tau(g)}(\alpha)\right\rangle .
$$

Proof. The following $F C$-modules are known to be isomorphic:

$$
\begin{gathered}
\operatorname{Hom}(V \otimes W, V \otimes W), \quad(V \otimes W) \otimes(\hat{V}, \hat{W}), \quad(V \otimes \hat{V}) \otimes(W \otimes \hat{W}), \\
\operatorname{Hom}(V, V) \otimes \operatorname{Hom}(W, W), \quad \operatorname{Hom}(\operatorname{Hom}(V, V), \operatorname{Hom}(W, W)),
\end{gathered}
$$

where the latter isomorphism is due to the fact that $\operatorname{Hom}(W, W)$ is self-dual. This implies that $\mu_{0}^{(\rho \otimes \tau)(g)}(1)=d_{M}^{g}$ where $M=\operatorname{Hom}(\operatorname{Hom}(V, V), \operatorname{Hom}(W, W))$. By Lemma 2.19, $d_{M}^{g}=\sum_{\alpha}\left\langle\mu^{g}(\alpha), \mu^{\tau(g)}(\alpha)\right\rangle$.

LEMMA 2.25. $\mu_{0}^{(\rho \otimes \tau)(g)}(1) \leqslant n^{2} \mu_{0}^{\tau(g)}(1)$.

Proof. By Lemmas 2.24 and 2.23

$$
\mu_{0}^{(\rho \otimes \tau)(g)}(1)=\sum_{\alpha}\left\langle\mu^{g}(\alpha), \mu^{\tau(g)}(\alpha)\right\rangle \leqslant \sum_{\alpha} \mu^{g}(\alpha) \mu_{0}^{\tau(g)}(1) .
$$

As $\sum_{\alpha} \mu^{g}(\alpha)=n^{2}$, the lemma follows.

TheOREm 2.26. Let $\rho, \tau$ be non-equivalent irreducible representations of a group $G=\left\langle g_{1}, \ldots, g_{k}\right\rangle$ and $g_{k+1}=g_{1} \cdots g_{k}$. Suppose that $\rho \otimes \tau$ is rigid. Then $\rho$ and $\tau$ are rigid.

Proof. Let $n=\operatorname{dim} \rho$ and $n^{\prime}=\operatorname{dim} \tau$. Then

$$
(k-1) n^{2}\left(n^{\prime}\right)^{2}+2=\sum_{j=1}^{k+1} \mu_{0}^{(\rho \otimes \tau)\left(g_{j}\right)}(1) \leqslant n^{2} \sum_{j=1}^{k+1} \mu_{0}^{\tau\left(g_{j}\right)}(1)
$$

by Lemma 2.25 , whence $n^{2}\left((k-1)\left(n^{\prime}\right)^{2}-\sum_{j=1}^{k+1} \mu_{0}^{\tau\left(g_{j}\right)}(1)\right) \leqslant-2$. Therefore,

$$
\left(n^{\prime}\right)^{2}(k-1)-\sum_{j=1}^{k+1} \mu_{0}^{\tau\left(g_{j}\right)}(1)<0 .
$$


The left-hand side is even (Lemma 2.20), and is not less than -2 by Lemma 2.6 as $\tau$ is irreducible. So the equality holds, which means that $\tau$ is rigid. By symmetry, so is $\rho$.

Lemma 2.27. Suppose that $g \in \mathrm{GL}(n, F)$ preserves a non-degenerate bilinear form $f$.

(1) $g$ is real; in addition, if $n$ is odd and $\operatorname{det} g=1$ then 1 is an eigenvalue of $g$.

(2) Suppose that $g$ is semisimple; then $n-d^{g}$ is even.

(3) Suppose that $g$ is unipotent and $\operatorname{Jord} g=\operatorname{diag}\left(k_{1} J_{1}, k_{2} J_{2}, \ldots, k_{r} J_{r}\right)$. If $f$ is alternating then $k_{i}$ are even for $i$ odd; if $p \neq 2$ and $f$ is symmetric then $k_{i}$ are even for $i$ even.

Proof. (1) Let $X$ be the Gram matrix of $f$ associated to the standard basis in $F^{n}$, and let $g^{T}$ denote the transpose of $g$. Then $g^{T} X g=X$. As $X$ is non-degenerate, it follows that $g$ is similar to $\left(g^{T}\right)^{-1}$. It is well known that $g$ and $g^{T}$ are similar matrices. Hence $\operatorname{det}(g-\mathrm{Id})=\operatorname{det}\left(g^{-1}-\mathrm{Id}\right)=\operatorname{det}(\mathrm{Id}-g) \operatorname{det} g^{-1}$ as $\operatorname{det} g=1$. If $n$ is odd, $\operatorname{det}(g-\mathrm{Id})=0$ and the result follows.

(2) Set $W=V^{g}$, that is, $W$ is the 1-eigenspace for $g$ and $d^{g}=\operatorname{dim} W$. Then $W$ is non-degenerate, that is, $W \cap W^{\perp}=0$ so $g$ fixes no non-zero vector on $W^{\perp}$. By (1), $\operatorname{dim} W^{\perp}$ is even, whence the result.

(3) See [20, Chapter IV-E.2.10].

Theorem 2.28. Let $q$ be even, $H=H_{237}$ and let $\phi: H \rightarrow \operatorname{Sp}(n, q)$ be an irreducible representation. Then $n \neq 10$. If $n=8,12$ or 16 then $\phi(H)$ preserves a quadratic form. In particular, groups $\operatorname{Sp}(8, q), \operatorname{Sp}(10, q), \Omega^{ \pm}(10, q), \operatorname{Sp}(12, q)$ and $\operatorname{Sp}(16, q)$ with $q$ even are not Hurwitz.

Proof. Suppose the contrary. By Lemma 2.27, elements $\phi(y)$ and $\phi(x y)$ are real. Therefore, the multiplicity vector for $\phi(x), \phi(y), \phi(x y)$ can be expressed as $[a, n-a]$ $[n-2 b, b, b]\left[m_{0}, m_{1}, m_{2}, m_{3}, m_{3}, m_{2}, m_{1}\right]$. Recall that $2 d_{S}^{\phi(x)}=n(n+1)-2 a(n-a)$ whose minimum value is attained by $a=n / 2$ and is equal to $n(n+2) / 2$. Similarly, $2 d_{S}^{\phi(y)}=n(n+1)-2 b(2 n+1)+6 b^{2}$ and $2 d_{S}^{\phi(x y)}=\left(n-2 m_{1}-2 m_{2}-2 m_{3}\right)\left(n-2 m_{1}-\right.$ $\left.2 m_{2}-2 m_{3}+1\right)+2 m_{1}^{2}+2 m_{2}^{2}+2 m_{3}^{2}$. One can check that the minimum values for $d_{S}^{\phi(y)}$ and $d_{S}^{\phi(x y)}$ are $(12,6),(19,9),(26,12),(46,20)$ for $n=8,10,12,16$, respectively. It follows that that the minimum of $d f_{S}^{\phi}=\operatorname{dim} S-d_{S}^{\phi(x)}-d_{S}^{\phi(y)}-d_{S}^{\phi(x y)}$ is equal to $-2,-3,-2,-2$, respectively. If $n=10$ then $d f_{S}=-3$ which violates Proposition $2.18(3)$. If $n=8,12,16$, this contradicts Proposition 2.18(3).

Recall that $J_{k}$ denotes the Jordan block of size $k$ with eigenvalue 1.

Lemma 2.29. Let $l, m$ be integers such that $1 \leqslant l \leqslant m \leqslant p$. The Jordan form of $J_{l} \otimes J_{m}$ is described as follows.

(i) If $l+m \leqslant p$ then $\operatorname{Jord}\left(J_{l} \otimes J_{m}\right)=\operatorname{diag}\left(J_{m+l-1}, J_{m+l-3}, \ldots, J_{m-l+1}\right)$;

(ii) If $l+m>p$ and $m<p$ then

$$
\operatorname{Jord}\left(J_{l} \otimes J_{m}\right)=\operatorname{diag}\left(J_{p}, \ldots, J_{p}, J_{2 p-m-l-1}, J_{2 p-l-m-3}, \ldots, J_{m-l+1}\right),
$$

where $J_{p}$ is repeated $m+l-p$ times;

(iii) If $m=p$ then $\operatorname{Jord}\left(J_{l} \otimes J_{p}\right)=\operatorname{diag}\left(J_{p}, \ldots, J_{p}\right)$, where $J_{p}$ is repeated $l$ times.

Proof. See [7, Theorem VIII.2.7]. 


\section{Examples of rigid representations}

In this section we denote by $\tilde{H}$ the group defined by two generators $\tilde{x}, y$ subject to relations $\tilde{x}^{4}=\left[\tilde{x}^{2}, y\right]=y^{3}=(\tilde{x} y)^{7}=1$. Then the mapping $\tilde{x} \rightarrow x, y \rightarrow y$ extends to a homomorphism $\tilde{H} \rightarrow H_{237}$. The kernel of this homomorphism is $Z(\tilde{H})$ (it is well known that $Z(H)=1$ ).

As above, $F$ is an algebraically closed field of characteristic $p$. If $p \neq 3$ then we fix a primitive 3 -root $\omega$ of 1 , and if $p \neq 7$ then we fix some primitive 7 -root $\varepsilon$ of 1 . Recall that $\bar{p}=p$ if $\left(7, p^{3}-p\right) \neq 1$ and $\bar{p}=p^{3}$ otherwise.

We shall often use the following fact without a reference.

LEMma 3.1. (1) Group $\tilde{H}$ (and $H_{237}$ ) coincides with its commutator subgroup. This is also true for every Hurwitz group.

(2) If $\phi$ is a linear representation of $\tilde{H}$ (or a Hurwitz group), then all matrices in the image of $\phi$ have determinant 1.

Lemma 3.2. Let $G=\langle x, y\rangle \in \mathrm{GL}(n, F)$ be irreducible and $x^{2} \in Z(G), y^{3} \in Z(G)$. Then the dimension of each eigenspace of $y$ does not exceed $n / 2$, while the dimension of each eigenspace of $x$ does not exceed $2 n / 3$.

Proof. Let $W$ be an eigenspace of $x$. Then $W \cap y W \cap y^{2} W=0$ as it is $G$-stable. It follows that $\operatorname{dim} W \leqslant 2 n / 3$. Similarly, if $W$ is an eigenspace of $y$, then $W \cap x W=0$, hence $\operatorname{dim} W \leqslant n / 2$.

LEMMA 3.3. (1) If $p \neq 7$ then there are exactly 3 equivalence classes of irreducible representations $\tilde{H} \rightarrow \mathrm{GL}(2, F)$.

(2) If $p=7$ then all irreducible representations $\tilde{H} \rightarrow \mathrm{GL}(2, F)$ are equivalent.

(3) Let $\phi: \tilde{H} \rightarrow \mathrm{GL}(2, F)$ be an irreducible representation and $p>0$. Then $\phi(\tilde{H}) \cong \mathrm{SL}(2, \bar{p})$.

Proof. Let $p \neq 7$. Let $C_{1}, C_{2}, C_{\varepsilon}$ be the similarity classes of matrices $\operatorname{diag}(i,-i)$, $\operatorname{diag}\left(\omega, \omega^{-1}\right)$ and $\operatorname{diag}\left(\varepsilon, \varepsilon^{-1}\right)$, respectively. It is well known and can be easily observed that there are matrices $A \in C_{1}, B \in C_{2}$ such that $A B \in C_{\varepsilon}$. Then the mapping $\tilde{x} \rightarrow A, y \in B$ extends to a homomorphism $\lambda_{\varepsilon}: \tilde{H} \rightarrow \operatorname{GL}(2, F)$. By Lemma 3.1, $\lambda_{\varepsilon}$ is irreducible. Obviously, $\lambda_{\varepsilon}$ is rigid so it is unique up to equivalence (Theorem 2.9). In addition, $\lambda_{\varepsilon}, \lambda_{\varepsilon^{2}}$ and $\lambda_{\varepsilon^{3}}$ are pairwise non-equivalent as their characters are distinct. Let $\lambda$ be an arbitrary irreducible representation of $\tilde{H}$ in $\operatorname{GL}(2, F)$. Then $\lambda(\tilde{H}) \in \mathrm{SL}(2, F)$ by Lemma 3.1. Hence $\lambda(\tilde{x} y)$ belongs to $C_{\varepsilon}, C_{\varepsilon^{2}}$ or $C_{\varepsilon^{3}}$, and $\lambda(\tilde{x}) \in C_{1}, \lambda(y) \in C_{2}$. By Theorem 2.9, $\lambda$ is equivalent to $\lambda_{\varepsilon}, \lambda_{\varepsilon^{2}}$ or $\lambda_{\varepsilon^{3}}$.

(2) In this case let $C_{3}$ be the similarity class containing $J_{2}$. A similar argument works. As there is a single similarity class of elements of order 7 in $\operatorname{GL}(2, F)$, all irreducible representations $\tilde{H} \rightarrow \mathrm{GL}(2, F)$ are equivalent.

(3) By Theorem 2.11, $\lambda(\tilde{H})$ is similar to a subgroup of $\operatorname{SL}(2, \bar{p})$. Using the classification of finite groups of $(2 \times 2)$-matrices (consult, for instance [8]) one observes that $\mathrm{SL}(2, \bar{p})$ contains no proper irreducible subgroups containing an element of order 7 .

Remarks. (1) Item (3) in Lemma 3.3 is a theorem of Macbeath [14].

(2) Below we hold symbols $\lambda_{\varepsilon}, \lambda_{\varepsilon^{2}}$ and $\lambda_{\varepsilon^{3}}$ to denote the representations in item (1) of the lemma. 
LemmA 3.4. Let $G=\mathrm{SL}\left(2, p^{m}\right)$ and let $\rho_{n}$ denote the natural $F$-representation of $G$ in the space of homogeneous polynomials in two variables of degree $n-1$ (so $\left.\operatorname{dim} \rho_{n}=n\right)$. Assume $2 \leqslant n \leqslant p$.

(1) $\rho_{n}$ is irreducible.

(2) $\rho_{n}(G)$ preserves a symmetric bilinear form if $n$ is odd, otherwise it preserves a skew symmetric bilinear form.

(3) Let $\gamma$ denote the Frobenius (or field) automorphism of $\operatorname{SL}\left(2, p^{m}\right)$ obtained from the mapping $y \rightarrow y^{p}$ for $y \in F_{p^{m}}$. Let $1 \leqslant k<m$. Then $\rho_{i} \otimes \gamma^{k} \rho_{j}$ is irreducible.

Proof. This is well known. (1) and (3) is a particular case of Steinberg [18, Theorem $49])$. As every element of $\mathrm{SL}(2, F)$ conjugate to its inverse, the characters of $\rho_{n}$ and of its dual coincide. Hence $\rho_{n}$ is self-dual. As $\rho_{n}(\mathrm{Id})=\operatorname{Id}$ for $n$ odd, (3) follows from [18, Lemma 79].

Lemma 3.5. Let $G=\mathrm{GL}(2, F)$. Let $\rho_{n}$ denote the natural F-representation of $G$ in the space of homogeneous polynomials of degree $n-1\left(\right.$ so $\left.\operatorname{dim} \rho_{n}=n\right)$. Assume $2 \leqslant n \leqslant p$.

(1) The representation $\rho_{n}$ is irreducible.

(2) For $i=1,2,3$ let $\lambda_{\varepsilon^{i}}$ be the representation $\tilde{H} \rightarrow \mathrm{GL}(2, F)$ defined in Lemma 3.3. Then the representation $\rho_{n} \lambda_{\varepsilon^{i}}: \tilde{H} \rightarrow \mathrm{GL}(n, F)$ is irreducible. If $n<6$, it is rigid.

(3) For $p \neq 7$ and for each $n=3,4,5$ the representations $\rho_{n} \lambda_{\varepsilon^{i}}$ with $i=1,2,3$ are pairwise non-equivalent.

(4) If $n$ is odd, $\rho_{n} \lambda_{\varepsilon^{i}}$ is trivial on $\tilde{x}^{2}$ so it can be viewed as a representation of $H_{237}$.

Proof. By Lemma 3.4(1), $\rho_{n}(\mathrm{SL}(2, p))$ is irreducible for $n \leqslant p$. This implies (1). As $\lambda_{\varepsilon^{i}}(\tilde{H})=\mathrm{SL}(2, \bar{p}),(2)$ follows. Rigidity in (2) is a matter of a simple computation. (3) is implied by the fact that the character values of $\tilde{x} y$ for $i=1,2,3$ are distinct for each $n<6$. (4) is obvious.

REMARK. $\rho_{3}$ and $\rho_{5}$ can be viewed as representations of $\operatorname{PSL}(2, q)$.

Lemma 3.6. (1) Let $p \neq 7$ and $H=H_{237}$. Then $H$ has rigid representations $\phi_{1}, \phi_{2}$ of dimension 3 whose multiplicity vectors are

$$
[1,2][1,1,1][0,1,1,0,1,0,0] \text { and }[1,2][1,1,1][0,0,0,1,0,1,1] \text {, }
$$

respectively, if $p \neq 2$; otherwise,

$$
[2,1][1,1,1][0,1,1,0,1,0,0] \text { and }[2,1][1,1,1][0,0,0,1,0,1,1] \text {. }
$$

In addition, $\phi_{i}(H) \cong \operatorname{SL}(3,2) \cong \operatorname{PSL}(2,7)$ for $i=1,2$ and $\phi_{i}(H)$ preserves no symmetric bilinear form.

(2) Let $p \neq 2,7$. Then $\tilde{H}$ has rigid representations $\psi_{1}, \psi_{2}$ of dimension 4 with multiplicity vectors

$$
[0,2,0,2][2,1,1][1,1,1,0,1,0,0] \text { and }[0,2,0,2][2,1,1][1,0,0,1,0,1,1] \text {, }
$$

respectively. In addition, $\psi_{i}(\tilde{H}) \cong \mathrm{SL}(2,7)$ for $i=1,2$ and $\psi_{i}(\tilde{H})$ preserves no symmetric bilinear form. 
Proof. Let $G=\operatorname{SL}(2,7)$ and let $a, b \in G$ be such that $a^{2} \in Z(G) b^{3}=1$ and $(a b)^{7}=1$. Group $G$ has irreducible representations $\phi_{1}^{\prime}, \phi_{2}^{\prime}$ of dimension 3 , and $\psi_{1}^{\prime}$, $\psi_{2}^{\prime}$ in dimension 4 with the above multiplicity vectors for $a, b, a b$. This can be seen by inspection of the Brauer character table (see [1] and [2]) unless $a$ or $b$ is of order $p$. Let $\phi_{1}, \phi_{2}$ and $\psi_{1}, \psi_{2}$ be the representations of $\tilde{H}$ obtained from a surjective homomorphism $\tilde{H} \rightarrow \mathrm{SL}(2,7)$. If $p=3$ then $c^{\phi_{i}^{\prime}(b)} \leqslant 3$ and $c^{\psi_{i}^{\prime}(b)} \leqslant 6$ for $i=1,2$ by formula (11). This implies that $\operatorname{Jord} \phi_{i}^{\prime}(b)=J_{3}$ and $\operatorname{Jord} \psi_{i}^{\prime}(b)=\operatorname{diag}\left(J_{3}, 1\right)$ for $i=1,2$. The case $p=2$ occurs only in (1), where $\operatorname{diag}\left(1, J_{2}\right)$ is the only option for Jord $\psi^{\prime}(a)$. So the lemma follows.

Remarks. (1) The case $p=7$ is considered in Lemma 3.5.

(2) Recall that $\operatorname{Jord} \psi_{i}(\tilde{x})=\operatorname{diag}(i, i,-i,-i)$ where $i^{2}=-1$ and the multiplicity vector of $\psi_{i}(\tilde{x})$ is $[0,2,0,2]$ according to our convention.

Lemma 3.7. Let $p \neq 2,7$ and let $H=H_{237}$. Then $H$ has a rigid representation $\pi$ of degree 6 with multiplicity vector $[4,2][2,2,2][0,1,1,1,1,1,1]$. In addition, $\pi(H)$ preserves a symmetric bilinear form and $\pi(H) \cong \mathrm{SL}(3,2)$.

Proof. Let $G=\operatorname{SL}(3,2)$. If $p \neq 2,7$ then $G$ has an irreducible representation $\pi$ of degree 6 and $\pi(G)$ preserves a symmetric bilinear form; see [2]. As $G$ is a quotient group of $H, \pi$ can be regarded as a representation of $H$. If $p \neq 3$ then $\pi$ is not modular and the lemma follows by inspection of the character of $\pi$ in [1]. Let $p=3$ and let $S$ be a Sylow 3-subgroup of $G$. As $|S|=3$, a complex irreducible representation of degree 6 is of defect 0 , hence it remains irreducible under reduction modulo 3. The representation obtained is equivalent to $\pi$ as $\pi$ is unique. The restriction to $S$ of a modular representation of defect 0 is a direct sum of the regular representation of $S$. This means that $\operatorname{Jord} \pi(y)=\operatorname{diag}\left(J_{3}, J_{3}\right)$, and the result follows.

Lemma 3.8. Let $p \neq 2$ and let $H=H_{237}$. Then $H$ has a rigid representation $\theta$ of degree 7 with multiplicity vector $[3,4][1,3,3][1,1,1,1,1,1,1]$ if $p \neq 3$, and $[3,4][3,3,1][1,1,1,1,1,1,1]$ for $p=3$. In addition, $\sigma(H)$ preserves a symmetric bilinear form and $\theta(H) \cong \mathrm{SL}(2,8)$.

Proof. Let $G=\mathrm{SL}(2,8)$. If $p \neq 2,3$ then $G$ has an irreducible representation $\theta$ of degree 7 whose Brauer character value at elements of order 3 is equal to -2 ; see [2]. As $G$ is a quotient group of $H_{237}, \theta$ can be viewed as a representation of $H$. If $p \neq 3,7$ then $\theta$ is not modular so the lemma follows by inspection of the character of $\theta$ in [1]. Let $p=7$ and let $S$ be a Sylow 7-subgroup of $G$. As $|S|=7$, all four complex irreducible representations of degree 7 are of defect 0 . Hence $G$ has four 7 -modular irreducible representations of defect 0 . The restrictions to $S$ of each of them is the regular representation of $S$. It follows that $\operatorname{Jord} \theta(X Y)=J_{7}$ and the result follows.

Let $p=3$. Sylow 3 -subgroups of $G$ are cyclic of order 9 . Let $t \in G$ be of order 9. It follows from the theory of representations of groups with cyclic Sylow $p$-subgroup [7, Ch. VII] that Jord $\theta(t)=J_{7}$; see [29, Lemma 2.2]. Then Jord $\theta\left(t^{3}\right)=$ $\operatorname{diag}\left(J_{3}, J_{2}, J_{2}\right)$. As $y$ is conjugate to $t^{3}$ in $G$, the result follows.

The fact that $\theta$ preserves a symmetric bilinear form is recorded in [2].

REMARK. The group $G=\operatorname{SL}(2,8)$ has no irreducible representation of dimension 7 in characteristic 2 . 
Lemma 3.9. Assume $p \neq 3$ and let $H=H_{237}$. Then $H$ has rigid representations $\sigma_{1}, \sigma_{2}$ of degree 13 whose multiplicity vectors are $[7,6][4,6,3][1,2,2,2,2,2,2]$ and $[7,6][4,3,6][1,2,2,2,2,2,2]$, respectively, for $p \neq 7$; if $p=7$ then they are $[7,6][4,6,3][2,2,2,2,2,2,1]$ and $[7,6][4,3,6][2,2,2,2,2,2,1]$. In addition, $\sigma_{i}(H)$ for $i=1,2$ preserves no symmetric bilinear form and $\sigma_{i}(H) \cong \operatorname{PSL}(2,27)$.

Proof. Let $G=\operatorname{PSL}(2,27)$. Let $h: H_{237} \rightarrow G$ be a surjective homomorphism and let $X=h(x), Y=h(y)$. Suppose first that $F=\mathbf{C}$. Then $G$ has two irreducible representation $\sigma_{1}, \sigma_{2}$ of degree 13. Let $\chi_{1}, \chi_{2}$ be their characters. Reordering $\sigma_{1}$ and $\sigma_{2}$ if necessary, by [1] we observe that $\chi_{1}(X)=1, \chi_{1}(X Y)=-1$ and $\chi_{1}(Y)=\frac{1}{2}(-1+3 \sqrt{-3})=1+3 \omega, \chi_{1}\left(Y^{2}\right)=1+3 \omega^{2}$ where $\omega=\frac{1}{2}(-1+\sqrt{-3})$. In addition, $\chi_{2}\left(Y^{m}\right)=\chi_{1}\left(Y^{2 m}\right)$ for $m=1,2$ and the values of $\chi_{2}$ at $X$ and $X Y$ are the same. The eigenvalue multiplicities of of $X, Y$ and $X Y$ can be obtained from computations with the characters of cyclic groups $\langle X\rangle,\langle Y\rangle,\langle X Y\rangle$, respectively. This yields the result on multiplicity vectors. In fact, $G$ has an irreducible representation of dimension 13 in any characteristic not equal to 3 (see [2]), and the Brauer character values on $p^{\prime}$-elements of $G$ coincide with the complex character values. Therefore, the Jordan form of $p^{\prime}$-elements and their multiplicity vectors are the same as in the complex number case. Let $p=2$. The multiplicity vector for $X$ is of shape $[a, 13-a]$ where $a \geqslant 7$. So $c^{X}=a^{2}+(13-a)^{2} \geqslant 85$ with equality for $a=7$, and the value is greater for $a>7$. Then $c^{X}+c^{Y}+c^{X Y}=c^{X}+86 \geqslant 85+86=13^{2}+2$. Scott's formula implies that $a=7$.

Let $p=7$. Here $c^{X}+c^{Y}=146$ so Scott's formula implies that $c^{X Y} \leqslant 171-146=$ 25. As $|X Y|=7$, the Jordan form of $X Y$ has no block of size greater than 7 . If the multiplicity vector of $X Y$ is $[2,2,2,2,2,2,1]$ then $c^{X Y}=25$ and for all other multiplicity vectors this value is greater. Therefore, $[2,2,2,2,2,2,1]$ is the only option, and the rigidity follows. Clearly, Jord $X Y=\operatorname{diag}\left(J_{7}, J_{6}\right)$.

LEMMA 3.10. Let $G_{1}, G_{2}$ be finite simple groups and $X \subset G_{1} \times G_{2}$ a proper subgroup. Let $\pi_{i}: X \rightarrow G_{i}$ denote the natural projections. Suppose that $\pi_{i}(X)=G_{i}$. Then $X \cong G_{1} \cong G_{2}$ and $\pi_{2} \pi_{1}^{-1}: G_{1} \rightarrow G_{2}$ is an isomorphism $\alpha: G_{1} \rightarrow G_{2}$.

Proof. Set $K_{i}=\operatorname{ker} \pi_{i}$. Clearly, $K_{1} \cap K_{2}=1$ so $\pi_{1}\left(K_{2}\right) \cong K_{2}$. If $K_{2} \neq 1$ then $\pi_{1}\left(K_{2}\right)$ is a non-trivial normal subgroup of $G_{1}=\pi_{1}(X)$. Therefore, $K_{2}=1$ or $\pi_{1}\left(K_{2}\right)=G_{1}$. In the latter case $|X|=\left|G_{1}\right| \cdot\left|G_{2}\right|$ so $X=G_{1} \times G_{2}$. Therefore, $K_{2}=1$. Similarly, $K_{1}=1$. So $G_{1} \cong G_{2} \cong X$ and $\pi_{1}, \pi_{2}$ are isomorphisms. The second claim of the lemma is trivial.

LEMma 3.11. For $1 \leqslant i<j \leqslant 3$ let $\lambda_{\varepsilon^{i}}: \tilde{H}_{237} \rightarrow \mathrm{SL}(2, p)$ be as in Lemma 3.3. Define $\bar{\lambda}_{i}: H_{237} \rightarrow \operatorname{PSL}(2, p)$ to be $\lambda_{\varepsilon^{i}}$ followed by the projection $\operatorname{SL}(2, p) \rightarrow$ $\operatorname{PSL}(2, p)$. In Lemma 3.10 specify $G_{1} \cong G_{2} \cong \operatorname{PSL}(2, p)$ where $\left(p^{2}-1,7\right) \neq 1$ and $X=\left\{\left(\bar{\lambda}_{i}(h), \bar{\lambda}_{j}(h)\right): h \in H_{237}\right\}$. Then $X=G_{1} \times G_{2}$.

Proof. The lemma follows from Lemma 3.10 as soon as we show that $\tau:=\bar{\lambda}_{j} \bar{\lambda}_{i}^{-1}$ is not an isomorphism. Let $C_{i}$ be the conjugacy class in $\operatorname{PSL}(2, p)$ corresponding to the matrices $\operatorname{diag}\left(\varepsilon^{i}, \varepsilon^{-i}\right)$ for $1 \leqslant i \leqslant 3$. It is well known that every automorphism $\alpha$ of $\operatorname{PSL}(2, p)$ is obtained from an inner automorphism of $\operatorname{PGL}(2, p)$. Hence $\alpha$ preserves $C_{i}$ in contrast with $\tau$. So the result follows. 
LEMMA 3.12. For $i=1,2$ let $p \neq 7$ and let $p \geqslant n_{i}>1$ be integers. Set $\alpha_{1}=\rho_{n_{1}} \lambda_{\varepsilon^{j}}$ and $\alpha_{2}=\rho_{n_{2}} \lambda_{\varepsilon^{k}}$ where $1 \leqslant j<k \leqslant 3$ and $\lambda_{\varepsilon^{j}}, \lambda_{\varepsilon^{k}}$ are as in Lemma 3.3. Then $\beta:=\alpha_{1} \otimes \alpha_{2}$ is an irreducible representation of $\tilde{H}$. In addition, if $G:=\beta(\tilde{H})$ and $\bar{G}=G / Z(G)$ then $\bar{G} \cong \operatorname{PSL}\left(2, p^{3}\right)$ if $\bar{p}=p^{3}$ and $\bar{G} \cong \operatorname{PSL}(2, p) \times \operatorname{PSL}(2, p)$ otherwise.

Proof. As $\alpha_{i}(\tilde{H}) / Z\left(\alpha_{i}(\tilde{H})\right)=\operatorname{PSL}(2, \bar{p})$, one observes that $\beta(\tilde{H}) / Z(\beta(\tilde{H}))$ is contained in the direct product $\operatorname{PSL}(2, \bar{p}) \times \operatorname{PSL}(2, \bar{p})$. Suppose first that $\bar{p}=p$. Observe that $\alpha_{i}(\tilde{H})$ can be viewed as $\rho_{n_{i}}\left(G_{i}\right)$ where $G_{i}=\mathrm{SL}(2, p)$. Then $\beta(\tilde{H})$ is a homomorphic image of $C_{1} \times G_{2}$ by Lemma 3.11. So $\beta(\tilde{H})$ can be obtained as an external tensor product of irreducible representations of $G_{1}$ and $G_{2}$. It is well known that such a representation is irreducible.

Let $\bar{p}=p^{3}$. Let $\gamma$ denote the Frobenius (or field) automorphism of $\operatorname{SL}\left(2, p^{3}\right.$ ) obtained from the mapping $y \rightarrow y^{p}$ for $y \in F_{p^{3}}$. Then $\lambda_{\varepsilon^{k}}=\gamma^{a} \lambda_{\varepsilon^{j}}$ for some $a \in$ $\{1,2\}$ so the kernels of these representations coincide. Hence $\beta$ is a representation of $\operatorname{SL}\left(2, p^{3}\right)$ inflated to $\tilde{H}$. It is irreducible (see, for instance Steinberg $[\mathbf{1 8}$, Theorem $49])$ and $\beta(\tilde{H}) / Z(\beta(\tilde{H})) \cong \operatorname{PSL}\left(2, p^{3}\right)$.

We say that $G$ is a central product of groups $G_{1}, G_{2}$ if $G \cong\left(G_{1} \times G_{2}\right) / Z$ where $Z$ is a subgroup of $Z\left(G_{1} \times G_{2}\right)$. Below, we use this term only if $G$ is not a direct product of non-trivial subgroups.

LEMma 3.13. Assume $p \neq 7$ and let $\lambda_{\varepsilon}, \lambda_{\varepsilon^{2}}$, and $\lambda_{\varepsilon^{3}}$ be representations of $\tilde{H}$ introduced in the proof of Lemma 3.3. For $1 \leqslant i<j \leqslant 3$ set $\lambda_{i j}=\lambda_{\varepsilon^{i}} \otimes \lambda_{\varepsilon^{j}}$. Then $\lambda_{i j}$ are ordinary irreducible representations of $H_{237}$. They are rigid and nonequivalent to each other, and each $\lambda_{i j}$ preserves a symmetric bilinear form. In addition, $\lambda_{i j}(H) \cong \operatorname{PSL}\left(2, p^{3}\right)$ if $\bar{p}=p^{3}$ and $\lambda_{i j} \cong \operatorname{SL}(2, p) \circ \operatorname{SL}(2, p)$ (a central product) otherwise.

Proof. Observe that $\lambda_{i j}\left(\tilde{x}^{2}\right)=\mathrm{Id}$. Therefore $\lambda_{i j}$ can be viewed as a representation of $H_{237}$. We show first that these representations are irreducible. Suppose the contrary. It is easy to see that the eigenvalues of $\lambda_{i j}(\tilde{x} y)$ are $\varepsilon^{ \pm i \pm j} \neq 1$. Therefore, in view of Lemma 3.1, $\lambda_{i j}$ has no trivial composition factor. If $p \neq 2, \lambda_{i j}$ has no composition factor of dimension 2 as $H_{237}$ has no irreducible representation of dimension 2; see Lemma 3.3. Let $p=2$. Observe that the Jordan form of $y$ in each representation is $\operatorname{diag}\left(1,1, \omega, \omega^{-1}\right)$. If $\alpha, \beta$ are the composition factors then $\operatorname{det} \alpha(y)=1$ and $\operatorname{det} \beta(y)=1$, hence either $\alpha(y)=\mathrm{Id}$ or $\beta(y)=\mathrm{Id}$. This is impossible, by Lemma 3.1. As the characters of $\lambda_{i j}$ are distinct, they are not equivalent. The multiplicity vector $v$ of $\lambda_{12}$ is $[2,2][2,1,1][0,1,0,1,1,0,1]$ so the rigidity follows as $\langle v, v\rangle=18$. Other cases are similar. Choosing a suitable basis in $F^{2}$ one can assume that $\lambda_{\varepsilon^{i}}$ preserves a skew symmetric bilinear form with Gram matrix $\Gamma=\left(\begin{array}{cc}0 & 1 \\ -1 & 0\end{array}\right)$ provided that $p \neq 2$. Then $\lambda_{\varepsilon^{i}} \otimes \lambda_{\varepsilon^{j}}$ preserves a symmetric bilinear form with Gram matrix $\Gamma \otimes \Gamma$. If $p=2$, replace -1 by 1 with the same conclusion.

The additional claim follows from Lemma 3.12.

LEMMA 3.14. Let $H=H_{237}$.

(1) Let $p \neq 2,3,7$. Then $H$ has rigid $F$-representations $\nu_{1}, \nu_{2}, \nu_{3}$ of degree 8 with multiplicity vectors

$$
\begin{gathered}
{[4,4][2,3,3][0,2,1,1,1,1,2], \quad[4,4][2,3,3][0,1,2,1,1,2,1],} \\
{[4,4][2,3,3][0,1,1,2,2,1,1],}
\end{gathered}
$$


respectively. In addition, $\nu_{i}(H)$ preserves a symmetric bilinear form and $\nu_{i}(H) \cong$ $\mathrm{SL}(2, \bar{p})$ if $p \neq \bar{p}$ and a central product $\mathrm{SL}(2, p) \circ \mathrm{SL}(2, p)$ otherwise.

(2) Let $p \neq 2,7$. Then $H$ has rigid $F$-representations $\tau_{i j}(1 \leqslant i \leqslant 3, j=1,2)$ of degree 8 with multiplicity vectors

$$
\begin{array}{ll}
{[4,4][2,3,3][1,2,1,2,0,1,1],} & {[4,4][2,3,3][1,0,2,1,1,1,2],} \\
{[4,4][2,3,3][1,1,0,1,2,2,1],} & {[4,4][2,3,3][1,1,1,0,2,1,2],} \\
{[4,4][2,3,3][1,2,1,1,1,2,0],} & {[4,4][2,3,3][1,1,2,2,1,0,1] .}
\end{array}
$$

(If $p=3$, one has to replace $[2,3,3]$ by $[3,3,2]$.) In addition, $\tau_{i j}(H) \cong \operatorname{PSL}(2, \bar{p})$ $\circ \mathrm{SL}(2,7)$ (a central product) and $\tau_{i j}(H)$ preserves no symmetric bilinear form.

(3) Let $p=2$. Group $H$ has rigid F-representations $\sigma_{i j}(1 \leqslant i \leqslant 3, j=1,2)$ of degree 6 with multiplicity vectors

$$
\begin{array}{ll}
{[3,3][2,2,2][1,1,1,2,0,1,0],} & {[3,3][2,2,2][1,0,1,0,2,1,1],} \\
{[3,3][2,2,2][1,0,1,1,1,0,2],} & {[3,3][2,2,2][1,2,0,1,1,1,0],} \\
{[3,3][2,2,2][1,1,0,0,1,2,1],} & {[3,3][2,2,2][1,1,2,1,0,0,1] .}
\end{array}
$$

In addition, $\sigma_{i j}(H) \cong \mathrm{SL}(2,8) \times \mathrm{SL}(3,2)$ and $\sigma_{i j}(H)$ preserves no non-zero bilinear form.

Proof. (1) Set $\nu_{i}=\rho_{4} \lambda_{i} \otimes \lambda_{j}$ where $1 \leqslant i \leqslant 3$ and with $j=i+1(\bmod 3)$. Then $\nu_{i}(Z(\tilde{H}))=$ Id so $\nu_{i}$ can be viewed as a representation of $H$. The multiplicity vector of the representation $\nu_{i}$ is computed straightforward. The assertion on the structure of $\nu_{i}(\tilde{H})$ follows from Lemma 3.12. By Lemma 3.4, $\rho_{4} \lambda_{i}(\tilde{H})$ and $\lambda_{j}(\tilde{H})$ preserves a skew symmetric bilinear forms with matrices $\Gamma_{1}, \Gamma_{2}$, say. Then $\nu_{i}$ preserves a symmetric bilinear forms with matrix $\Gamma_{1} \otimes \Gamma_{2}$.

(2) Let $\lambda_{\varepsilon^{i}}(1 \leqslant i \leqslant 3)$ and $\psi_{j}(1 \leqslant j \leqslant 2)$ be representations defined in Lemmas 3.3 and 3.6, respectively. Let $G_{1} \cong \operatorname{SL}(2, \bar{p})$ and $G_{2} \cong \operatorname{SL}(2,7)$. Then $\lambda_{\varepsilon^{i}}(\tilde{H}) \cong G_{1}$ and $\psi_{j}(\tilde{H}) \cong G_{2}$. Set $\tau_{i j}=\lambda_{\varepsilon^{i}} \otimes \psi_{j}$ so $\operatorname{dim} \tau_{i j}=8$. Then $\tau_{i j}(\tilde{H})$ is contained in a quotient group of $G_{1} \times G_{2}$. In fact, $\lambda_{\varepsilon^{i}}$ can be viewed as a representation of $G_{1}$ and $\psi_{j}$ as a representation of $G_{2}$. Therefore, $\tau_{i j}(\tilde{H})$ is contained in the external tensor product $\lambda_{\varepsilon^{i}} \otimes \psi_{j}$ viewed as a representation of $G_{1} \times G_{2}$. This is well known to be irreducible. The image $G=:\left(\lambda_{\varepsilon^{i}} \otimes \psi_{j}\right)\left(G_{1} \times G_{2}\right)$ is isomorphic to $G_{1} \circ G_{2}$, a central product of these groups, as the center of $G$ is of order 2 and $G$ has subgroups isomorphic to $G_{1}$ and $G_{2}$.

As $\tau_{i j}\left(\tilde{x}^{2}\right)$ is the identity matrix, $\tau_{i j}$ is actually a representation of $H_{237}$. It is not hard to observe that $G_{1} \circ G_{2}$ has no proper Hurwitz subgroup. So the claim on images follows. The shape of the multiplicity vectors is the matter of a simple computation unless $p=3$. In this case $\operatorname{Jord} \lambda_{i}(y)=J_{2}$ and $\operatorname{Jord} \psi(y)=\operatorname{diag}\left(J_{1}, J_{3}\right)$. Then $\operatorname{Jord} \tau_{i j}(y)=\operatorname{diag}\left(J_{3}, J_{3}, J_{2}\right)$. This corresponds to the multiplicity vector $[3,3,2]$. So the rigidity index of $\tau_{i j}$ is equal to 0 (see Definition 2.8) hence $\tau_{i j}$ is rigid. Obviously, $\tau_{i j}$ is not self-dual, hence $\tau_{i j}(H)$ preserves no non-zero bilinear form.

(3) As in (2), set $\sigma_{i j}=\lambda_{\varepsilon^{i}} \otimes \phi_{j}$ where $\phi_{j}$ for $1 \leqslant j \leqslant 2$ is introduced in Lemma 3.6. The same argument as in (2) yields the result. 
Lemma 3.15. Let $p \neq 2,7$. Then $H_{237}$ has rigid representations $\eta_{m}(1 \leqslant m \leqslant 6)$ of dimension 9 with multiplicity vectors

$$
\begin{aligned}
{[5,4][3,3,3][1,2,2,2,1,1,0], } & {[5,4][3,3,3][1,1,2,1,2,0,2], } \\
{[5,4][3,3,3][1,1,2,2,0,1,2], } & {[5,4][3,3,3][1,2,0,2,1,2,1], } \\
{[5,4][3,3,3],[1,2,1,0,2,2,1], } & {[5,4][3,3,3],[1,0,1,1,2,2,2] . }
\end{aligned}
$$

In addition, the image of each representation preserves no symmetric bilinear form and it is isomorphic to $\operatorname{PSL}(2, \bar{p}) \times \mathrm{SL}(3,2)$.

Proof. We set $\eta_{i j}=\rho_{3} \lambda_{\varepsilon^{i}} \otimes \phi_{j}$ where $\rho_{3} \lambda_{\varepsilon^{i}}$ for $i=1,2,3$ are as in Lemma 3.5 and $\phi_{j}$ for $j=1,2$ are as in Lemma 3.6. Set $G_{1} \cong \operatorname{PSL}(2, \bar{p})$ and $G_{2} \cong \operatorname{SL}(3,2)$. As $\rho_{3} \lambda_{\varepsilon^{i}}(H) \cong G_{1}$ and $\phi_{j}(H) \cong G_{2}$, we see that $\eta_{i j}(H) \subseteq G_{1} \times G_{2}$. In fact, we have the equality here, as otherwise $\eta_{i j}(H)$ would be a proper Hurwitz subgroup of $G_{1} \times G_{2}$. These are only $G_{1}$ and $G_{2}$; however, $\eta_{i j}(H)$ is none of them.

If $p \neq 3$ then the shape of multiplicity vectors is the matter of elementary computation. If $p=3$ then the Jordan form of $\rho_{3} \lambda_{\varepsilon^{i}}(y)$ is $J_{3}$ as well as of $\phi_{j}(y)$. Therefore, Jord $\eta_{i j}(y)=\operatorname{Jord}\left(J_{3} \otimes J_{3}\right)$ which is known to be $\operatorname{diag}\left(J_{3}, J_{3}, J_{3}\right)$. The multiplicity vector for this matrix is $[3,3,3]$. So the rigidity index of $\eta_{i j}$ is equal to 0 (in the sense of Definition 2.8) hence $\eta_{i j}$ is rigid. As $\eta_{i j}$ is irreducible and not self-dual, $\eta_{i j}\left(H_{237}\right.$ preserves no non-zero bilinear form.

The results of this section are collected in Appendix A (Tables A-1, A-2, A-3, A-4). They are used to produce Tables B-1, B-2, B-3, B-4 (Appendix B) as follows. Let $\phi: H_{237} \rightarrow \mathrm{GL}(n, F)$ be an irreducible representation realized in a module $V$. Express the multiplicity vec- tor $m^{V}$ of $\phi(x), \phi(y), \phi(x y)$ as $[a, n-a],[n-b-$ $c, b, c],\left[m_{0}, m_{1}, m_{2}, m_{3}, m_{4}, m_{5}, m_{6}\right]$ where $m_{0}+\cdots+m_{7}=n$. Let $W$ be the module associated with a representation constructed in the above lemmas, and let $m^{W}$ be the corresponding multiplicity vector. Then we use formula (10) to produce the entries of Tables B-1, etc.

EXAMPLE. Let $W$ be the module associated with the representation in Lemma $3.6(1)$ for $p \neq 2,7$ so $m^{W}=[1,2][1,1,1][0,1,1,0,1,0,0]$. Then formula (10) gives:

$$
a+2(n-a)+(n-b-c)+b+c+m_{1}+m_{2}+m_{4} \leqslant 3 n
$$

which coincides with $T_{4}$ in Table B-1 and $T_{4}^{3}$ in Table B-3. If $p=2$ then $m^{W}=$ $[2,1][1,1,1][0,1,1,0,1,0,0]$ which similarly gives $T_{4}^{2}$ in Table B-2.

The condition in Tables B-1 to B-4 at the column headed 'warning' reminds the reader to be careful when the multiplicity vector on test is of the dimension indicated. One can use the test provided modules $V$ and $W$ are not isomorphic. To illustrate this, we show that the alternating group $A_{7}$ is not Hurwitz. Indeed, it has an irreducible complex representation of degree 6 and one can easily observe that the multiplicity vector of a triple of elements of order $2,3,7$ in $A_{7}$ can only be $[4,2][2,2,2][0,1,1,1,1,1,1]$. This contradicts $T_{12}$ which can be used for testing here as the test has been obtained from a representation $\theta$ with $\theta\left(H_{237}\right) \cong \operatorname{SL}(3,2)$.

We conclude this section by reminding the reader of some known examples of Hurwitz matrix groups of small dimensions. In particular, groups of Lie type $G_{2}(q)$ 
are Hurwitz for every $q>4$ (Malle [15]) as well as their twisted versions ${ }^{2} G_{2}(q)$ with $q=3^{2 m+1}$ and $m \geqslant 1$ (Jones [10]). They have 7-dimensional irreducible representations over the field of $q$ elements if $q$ is odd. If $q$ is even then $G_{2}(q)$ has 6-dimensional irreducible representations over the field of $q$ elements. In addition, groups ${ }^{3} D_{4}(q)$ with $q \neq 4$ and coprime to 3 are Hurwitz and they are realizable by $(8 \times 8)$-matrices over $F_{q^{3}}$ (see [16]). The representations of $H_{237}$ extending these representations are not basic (see the definition prior to Lemma 2.22). Therefore, they are useless for performing tensor tests. One can compute the multiplicity vectors of the representations in question. These are $[3,3],[2,2,2],[0,1,1,1,1,1,1]$ in dimension 6 with $p=2,[3,4],[3,2,2],[1,1,1,1,1,1,1]$ in dimension 7 and $[4,4]$, $[2,3,3],[2,1,1,1,1,1,1]$ in dimension 8 .

One can extract from [23] a list of sporadic simple groups known to be Hurwitz. These are $J_{1}, J_{2}, \mathrm{He}, \mathrm{Ru}, \mathrm{Co}_{3}, \mathrm{Fi}_{22}, \mathrm{HN}, \mathrm{Ly}, \mathrm{Th}, J_{4}, \mathrm{Fi}_{24}^{\prime}$, and $M$.

\section{Relationship between $d f_{A}, d f_{S}$ and $d f_{E}$}

Let $H=H_{237}$ and let $\phi: H \rightarrow \mathrm{GL}(n, F)$ be an irreducible representation. Set $G=\phi(H)$ and let $V$ be the associated $F H$-module. Of course, $V$ can be viewed as an $F G$-module. Express the multiplicity vector of the triple $\phi(x), \phi(y), \phi(x y)$ as follows:

$$
[a, n-a],[n-b-c, b, c],\left[m_{0}, m_{1}, m_{2}, m_{3}, m_{4}, m_{5}, m_{6}\right] .
$$

As in Section 2, $S$ denotes the set of symmetric matrices viewed as a $\operatorname{GL}(n, F)$ module via the congruence action $M \rightarrow g M g^{T}$ for $M \in S, g \in \mathrm{GL}(n, F)$. Similarly, $E$ is the set of skew symmetric matrices, if $p \neq 2$, and the set of symmetric matrices with zero diagonal if $p=2$. We denote by $A$ and $R$ the vector space $M(n, F)$ viewed as an $F G$-module under the adjoint and the congruence action, respectively. In other words, $A \cong V \otimes \hat{V}$ and $R \cong V \otimes V$. We view $S, E, A$ as $H$-modules obtained in the obvious way from $V$, so $d f_{S}^{H}, d f_{E}^{H}, d f_{A}^{H}$ are their defects defined prior to Lemma 2.2.

LEMMA 4.1.

$$
d f_{A}^{H}=: \quad-n^{2}-2 a^{2}+2 n(a+b+c)-2 b^{2}-2 c^{2}-2 b c-\sum_{i=0}^{6} m_{i}^{2} .
$$

Proof. This follows from the fact that $c^{\phi}(g)$ for $g \in H$ is equal to $\sum m_{i}^{2}$ where $m_{i}$ are the coordinates of the multiplicity vector for $\phi(g)$; see the comments prior to Lemma 2.19.

LEMma 4.2. Let $g \in \mathrm{GL}(n, F)$.

(1) Let $p \neq 2$ and $g^{2}=$ Id. Then $d_{A}^{g}=2 d_{S}^{g}-n$ and $d_{S}^{g}=d_{E}^{g}+n$.

(2) Let $p \neq 3, g^{3}=\mathrm{Id}$ and express the multiplicity vector of $g$ as $\left[d_{V}^{g}, b, c\right]$. Then $d_{A}^{g}=2 d_{S}^{g}-d_{V}^{g}+(b-c)^{2}$.

(3) Suppose that $p \neq 7, g^{7}=\mathrm{Id}$ and express the multiplicity vector of $g$ as $\left[d_{V}^{g}, m_{1}, m_{2}, m_{3}, m_{4}, m_{5}, m_{6}\right]$. Then $d_{A}^{g}=2 d_{S}^{g}-d_{V}^{g}+\left(m_{1}-m_{6}\right)^{2}+\left(m_{2}-m_{5}\right)^{2}+$ $\left(m_{3}-m_{4}\right)^{2}$.

Proof. (1) Let $a=d_{V}^{g}$. Then $d_{A}^{g}=a^{2}+(n-a)^{2}$ and $2 d_{S}^{g}=n^{2}+n-2 a(n-a)$. So the result follows.

(2) We have $d_{A}^{g}=\left(d_{V}^{g}\right)^{2}+b^{2}+c^{2}$ and $2 d_{S}^{g}=\left(d_{V}^{g}\right)^{2}+d_{V}^{g}+2 b c$, whence the result. 
(3) We have $d_{A}^{g}=\left(d_{V}^{g}\right)^{2}+\sum_{i=1}^{6} m_{i}^{2}$ and $2 d_{S}^{g}=\left(d_{V}^{g}\right)^{2}+d_{V}^{g}+2 m_{1} m_{6}+2 m_{2} m_{5}+$ $2 m_{3} m_{4}$, so the result follows.

LEMmA 4.3. Let $p=2$ and $g \in \operatorname{GL}(n, F)$. Let $\operatorname{Jord} g=\operatorname{diag}\left(k_{1} J_{1}, k_{2} J_{2}\right)$ and let $[a, n-a]$ be the multiplicity vector for $g$.

(1) $a=k_{1}+k_{2}, n-a=k_{2}$.

(2) $d_{S}^{g}=d_{E}^{g}+d_{V}^{g}=d_{E}^{g}+a$.

(3) $d_{S}^{g}=n(n+1) 2-a(n-a)$ and $d_{A}^{g}=2 d_{S}^{g}-n$.

Proof. (1) is trivial. (2) Let $B=\left\{b_{1}, \ldots, b_{n}\right\}$ be the standard basis of $F^{n}$. Then $g$ is similar to a permutation matrix $\pi$ (that is, $\pi B=B$ ), and the respective permutation of $B$ has $k_{2}$ cycles of size 2 and $k_{1}=2 a-n$ fixed points. Let $e_{i j}$ be the matrix with 1 at the $(i, j)$-position and 0 elsewhere. Then $\left\{e_{i j}+e_{j i}: 1 \leqslant\right.$ $i<j \leqslant n\}$ is a basis $B_{E}$ in $E$ and a basis $B_{S}$ of $S$ is obtained by adding $e_{i i}$ for $i=1, \ldots, n$. Then $\pi B_{E} \pi^{T}=B_{E}$ and $\pi B_{S} \pi^{T}=B_{S}$. Observe that $\pi^{T}=$ $\pi^{-1}$ so the action of $\pi$ on $\left\{e_{i i}\right\}$ is isomorphic to the action on $B$. This implies (3). It is well known that $\operatorname{dim} S^{g}$ is equal to the number of $\pi$-orbits on $B_{S}$. This number does not depend on the ground field so we can compute it by viewing the Jordan form of $\left.\pi\right|_{S}$ over Q. As $\operatorname{Jord}_{\mathbf{Q}} \pi=\operatorname{diag}\left(\operatorname{Id}_{a},-\operatorname{Id}_{n-a}\right)$ we have $\operatorname{dim} S^{g}=$ $(a(a+1)+(n-a)(n-a+1)) / 2=n(n+1) / 2-a(n-a)$, as required for $(2)$.

LEMMA 4.4. Let $g$ be a unipotent matrix in $\operatorname{GL}(n, F)$ and $p \neq 2$. Then $d_{A}^{g}=d_{S}^{g}+d_{E}^{g}$.

Proof. As $g$ is unipotent, $V$ and $\hat{V}$ are isomorphic $g$-modules. If $p \neq 2$ then $R=$ $S \oplus E$ is a direct sum of $\mathrm{GL}(n, F)$-modules with respect to the congruence action. Therefore, $d_{S}^{g}+d_{E}^{g}=d_{R}^{g}$. As $R \cong V \otimes V$ and $A \cong V \otimes \hat{V}$ and $g$ is unipotent, their restrictions to $g$ are isomorphic $g$-modules. Therefore, $d_{A}^{g}=d_{S}^{g}+d_{E}^{g}$.

LEMMA 4.5. Let $g \in \mathrm{GL}(n, F)$ and $g^{p}=\mathrm{Id}$.

(1) Let $p=3$ and Jord $g=\operatorname{diag}\left(k_{1} J_{1}, k_{2} J_{2}, k_{3} J_{3}\right)$. Then $d_{A}^{g}=2 d_{S}^{g}-d_{V}^{g}+k_{2}=$ $2 d_{S}^{g}-k_{1}-k_{3}$ and $d_{E}^{g}=d_{S}^{g}-d_{V}^{g}+k_{2}=d_{S}^{g}-k_{1}-k_{3}$.

(2) Let $p=7$ and $\operatorname{Jord} \phi(g)=\operatorname{diag}\left(k_{1} J_{1}, k_{2} J_{2}, k_{3} J_{3}, k_{4} J_{4}, k_{5} J_{5}, k_{6} J_{6}, k_{7} J_{7}\right)$. Then $d_{A}^{g}=2 d_{S}^{g}-k_{1}-k_{3}-k_{5}-k_{7}=2 d_{S}^{g}-d_{V}^{g}+k_{2}+k_{4}+k_{6}$.

Proof. (1) By [6, Lemma 4.3],

$$
d_{S}^{g}=\frac{k_{1}\left(k_{1}+1\right)}{2}+k_{1} k_{2}+k_{2}^{2}+\frac{k_{3}\left(3 k_{3}+1\right)}{2}+k_{1} k_{3}+2 k_{2} k_{3}
$$

On the other hand, $d_{A}^{g}=\left(k_{1}+k_{2}+k_{3}\right)^{2}+\left(k_{2}+k_{3}\right)^{2}+k_{3}^{2}$, so the result follows.

(2) By [6, Lemma 4.3],

$$
\begin{aligned}
2 d_{S}^{g}= & k_{1}\left(k_{1}+1\right)+2 k_{2}^{2}+k_{3}\left(3 k_{3}+1\right)+4 k_{4}^{2}+k_{5}\left(5 k_{5}+1\right)+6 k_{6}^{2}+k_{7}\left(7 k_{7}+1\right) \\
& +2 k_{1} \sum_{j=2}^{7} k_{j}+4 k_{2} \sum_{j=3}^{7} k_{j}+6 k_{3} \sum_{j=4}^{7} k_{j}+8 k_{4} \sum_{j=5}^{7} k_{j}+10 k_{5}\left(k_{6}+k_{7}\right)+12 k_{6} k_{7} .
\end{aligned}
$$

On the other hand, $d_{A}^{g}=\sum_{j=0}^{7}\left(\sum_{i=j}^{7} k_{i}\right)^{2}$. So the result follows by expansion of the above expressions. 
Proposition 4.6. (1) Let $p \neq 3,7$. Then

$d f_{A}^{H}=2 d f_{S}^{H}+d_{V}^{\phi(y)}+d_{V}^{\phi(x y)}-(b-c)^{2}-\left(m_{1}-m_{6}\right)^{2}-\left(m_{2}-m_{5}\right)^{2}-\left(m_{3}-m_{4}\right)^{2}$.

In particular, if $p \neq 3,7$ and $\phi(y)$ and $\phi(x y)$ are real then $d f_{A}^{H}=2 d f_{S}^{H}+d_{V}^{\phi(y)}+$ $d_{V}^{\phi(x y)}$.

(2) Let $p=2$. Then $d f_{S}^{H}=d f_{E}^{H}+d f_{V}^{H}$.

(3) Let $p \neq 2,3,7$. Then $d f_{E}^{H}=d f_{S}^{H}+d_{V}^{\phi(y)}+d_{V}^{\phi(x y)}$.

(4) Let $p=3$ and Jord $\phi(y)=\operatorname{diag}\left(k_{1} J_{1}, k_{2} J_{2}, k_{3} J_{3}\right)$. Then $d f_{A}^{H}=2 d f_{S}^{H}+k_{1}+$ $k_{3}+d_{V}^{\phi(x y)}-\left(m_{1}-m_{6}\right)^{2}-\left(m_{2}-m_{5}\right)^{2}-\left(m_{3}-m_{4}\right)^{2}$ and $d f_{E}^{\phi}=d f_{S}^{\phi}+d f_{V}^{\phi(x y)}+k_{1}+k_{3}$.

(5) Let $p=7$ and $\operatorname{Jord} \phi(y)=\operatorname{diag}\left(k_{1} J_{1}, k_{2} J_{2}, k_{3} J_{3}, k_{4} J_{4}, k_{5} J_{5}, k_{6} J_{6}, k_{7} J_{7}\right)$. Then $d f_{A}^{H}=2 d f_{S}^{H}+d_{V}^{\phi(y)}-(b-c)^{2}+k_{1}+k_{3}+k_{5}+k_{7}$ and $d f_{E}^{H}=d f_{S}^{H}+d f_{V}^{\phi(y)}+$ $k_{1}+k_{3}+k_{5}+k_{7}$.

Proof. (1) As $d f_{A}^{H}=n^{2}-d_{A}^{\phi(x)}-d_{A}^{\phi(y)}-d_{A}^{\phi(x y)}$ and $2 d f_{S}^{H}=n^{2}+n-2 d_{S}^{\phi(x)}-2 d_{S}^{\phi(y)}-$ $2 d_{S}^{\phi(x y)}$, the result for $p \neq 2$ follows from Lemma 4.2. If $p=2$, use additionally Lemma 4.3(3).

(2) By Lemma 4.3, $\operatorname{dim} S^{\phi(x)}=\operatorname{dim} E^{\phi(x)}+a$. In addition, $\operatorname{dim} S^{\phi(y)}=d_{V}^{\phi(y)}+$ $\operatorname{dim} E^{\phi(y)}$ and $\operatorname{dim} S^{\phi(x y)}=\operatorname{dim} E^{\phi(x y)}+d_{V}^{\phi(x y)}$. As $\operatorname{dim} S=\operatorname{dim} E+n$, we have that $d f_{S}^{H}=\operatorname{dim} S-d_{S}^{\phi(x)}-d_{S}^{\phi(y)}-d_{S}^{\phi(x y)}=\operatorname{dim} E+n-d_{E}^{\phi(x)}-d_{V}^{\phi(x)}-d_{E}^{\phi(y)}-$ $d_{V}^{\phi(y)}-d_{E}^{\phi(x y)}-d_{V}^{\phi(x y)}=d f_{E}^{H}+d f_{V}^{H}$.

(3) If $g \in G$ is of odd order coprime to $p$ then $d_{S}^{\phi(g)}=d_{E}^{\phi(g)}+d_{V}^{\phi(g)}$. As $d_{S}^{\phi(x)}=$ $d_{E}^{\phi(x)}+n$, the claim follows by straightforward computations.

(4), (5) Combine Lemmas 4.2 and 4.5.

REMARKS. (i) Claim (2) shows that test $T_{S}$ is useless for $p=2$, while the formulas for $E$ in items (3), (4), (5) tell us that $T_{E}$ is useless for $p \neq 2$.

(ii) Formulas in items (4) and (5) can be easily expressed in terms of multiplicity vectors. Say, if $p=3$ then $n-b-c=k_{1}+k_{2}+k_{3}, b=k_{2}+k_{3}$ and $c=k_{3}$ whence $k_{1}+k_{3}=n-2 b$. Similarly, if $p=7$ then $k_{1}+k_{3}+k_{5}+k_{7}=m_{0}-m_{1}+m_{2}-m_{3}+$ $m_{4}-m_{5}+m_{6}=n-2\left(m_{1}+m_{3}+m_{5}\right)$.

Proposition 4.7. Suppose that the minimum polynomial of $\phi(x y)$ is of degree at most 5 ; then $n<7$. Moreover, if $p \neq 2$ then $n<6$.

Proof. Set $X=\phi(x)$ and $Y=\phi(y)$. Observe first that if $n=x_{1}+\cdots+x_{k}$ (where $n$ is fixed) then $x_{1}^{2}+\cdots+x_{k}^{2} \geqslant n^{2} / k$. (This fact does not require $x_{1}, \ldots, x_{k}$ to be integers and can be therefore obtained by computing the minimum of the real variable function $f\left(x_{1}, \ldots, x_{k}\right)=x_{1}^{2}+\cdots+x_{k}^{2}$ subject to the condition that $\left.n=x_{1}+\cdots+x_{k}\right)$. Therefore, in formula $n^{2}+2 \geqslant c^{X}+c^{Y}+c^{X Y}$ we have $c^{X} \geqslant n^{2} / 2$, $c^{Y} \geqslant n^{2} / 3, c^{X Y} \geqslant n^{2} / 5$, so $n^{2}+2 \geqslant 31 n^{2} / 30$ whence $n^{2} \leqslant 60$ and $n<8$.

As $m_{i}$ are integers, for $n=7$ one obtains that $c^{X} \geqslant 25, c^{Y} \geqslant 17$ and $c^{X Y} \geqslant 11$, which sums to $53>7^{2}+2$. Similarly, if $n=6$ and $p \neq 2$, one obtains that $c^{X} \geqslant 20$, $c^{Y} \geqslant 12$ and $c^{X Y} \geqslant 8$, which sums to $40>6^{2}+2$.

Proposition 4.8. Let $p=7$ and $n>6$. Suppose that the minimum polynomial of $\phi(x y)$ is of degree 6 . Then $\phi(H)$ preserves a symmetric bilinear form and $n=12$. 
Proof. The Jordan form of $\phi(x y)$ has no block of size 7 , hence $m_{6}=0$. Recall that $m_{0} \geqslant m_{1} \geqslant m_{2} \geqslant m_{3} \geqslant m_{4} \geqslant m_{5}$. We use Table B-4. If $n>5$, from $T_{0}^{7}$ and $T_{2}^{7}$ we get $2 a+m_{0} \leqslant n+m_{5}$ so $2 a \leqslant n-m_{0}+m_{5} \leqslant n$. By $T_{1}^{7}$ we have $2\left(m_{0}+m_{1}+m_{2}\right) \leqslant 2 a$ so $2\left(m_{0}+m_{1}+m_{2}\right) \leqslant n=\sum_{i} m_{i}$ hence $m_{0}+m_{1}+m_{2} \leqslant m_{3}+m_{4}+m_{5}$. Therefore, $m_{0}=m_{1}=m_{2}=m_{3}=m_{4}=m_{5}=n / 6$ and $n=2 a$. By the determinant condition, $a$ is even so $n$ is divisible by 4 . Furthermore, $n-b-c \leqslant n / 3$ from $T_{0}^{7}$ and that $n-b-c \geqslant n / 3$ from $T_{2}^{7}$. Hence $d^{\phi(y)}=n / 3$. It follows that the Jordan form of $\phi(x y)$ is $\left(m J_{6}\right)$ where $n=6 m$. By Lemma 4.5, $2 d_{S}^{\phi(x y)}=d_{A}^{\phi(x y)}=n^{2} / 6$; hence $d_{S}^{\phi(x y)}=n^{2} / 12$. Then

$$
d f_{S}^{H}=\frac{n(n+1)}{2}-\frac{n(n+2)}{4}-\frac{n(n+3)}{18}-b c-\frac{n^{2}}{12}=\frac{n^{2}}{9}-\frac{n}{6}-b c .
$$

Observe that

$$
-b c=-\frac{(b+c)^{2}}{2}+\frac{b^{2}+c^{2}}{2}=-\frac{2 n^{2}}{9}-\frac{n^{2}}{18}+\frac{d_{A}^{\phi(y)}}{2}=-\frac{5 n^{2}}{18}+\frac{d_{A}^{\phi(y)}}{2} .
$$

In addition, $d_{A}^{\phi(y)} \leqslant n^{2}+2-d_{A}^{\phi(x)}-d_{A}^{\phi(x y)}=n^{2}+2-n^{2} / 2-n^{2} / 6=n^{2} / 3+2$. Altogether,

$$
d f_{S}^{H} \leqslant \frac{n^{2}}{9}-\frac{n}{6}-\frac{5 n^{2}}{18}+\frac{n^{2}}{6}+1=-\frac{n}{6}+1
$$

As $d f_{S}^{H} \geqslant-2$, we conclude that $n \leqslant 18$. As $n$ is divisible by 12 , we have $n=12$. If $\phi(H)$ preserves no symmetric bilinear form, $-n / 6+1 \geqslant d f_{S}^{H} \geqslant 0$, which is impossible.

For $p>7$ we have only a weaker analogue of Proposition 4.8 .

Proposition 4.9. Suppose that $n>6, p \neq 7$ and $d_{V}^{\phi(x y)}=0$. Then either $n=12$ and $\phi(H)$ preserves a symmetric bilinear form, or $n=8, p \neq 2,3,7$ and $\phi$ is as in Lemma 3.14(1).

Proof. We use tests from Appendix B. Assume first that $p \neq 2,3,7$. By $T_{12}$ in Table B-1, $2 a \leqslant n$. If $n \neq 3$ then adding $T_{4}$ to $T_{5}$ we have that $\sum_{i>0} m_{i}=n \leqslant 2 a$. Hence $n=2 a$. As $n-a$ is even by the determinant condition, $n$ is divisible by 4 . In addition, $n=\left(m_{1}+m_{2}+m_{4}\right)+\left(m_{3}+m_{5}+m_{6}\right)$ implies $m_{1}+m_{2}+m_{4}=n / 2$ and $m_{3}+m_{5}+m_{6}=n / 2$ in view of $T_{4}, T_{5}$. Observe that $T_{6}, T_{7}$ and $T_{8}$ are equivalent to $n-b-c \leqslant m_{i}+m_{7-i}$ for $i=1,2,3$ (as $m_{0}=0$ ). Similarly, $T_{14}, T_{15}$ and $T_{16}$ are equivalent to $m_{i}+m_{7-i} \leqslant n-b-c$ (here we do not need to assume $n \neq 8$ as $\phi$ is not equivalent to the representations in Lemma 3.14(1)). Hence $m_{i}+m_{7-i}=n-b-c$. Summing these over $i \in\{1,2,4\}$, one obtains $3 b+3 c=2 n$, in particular, $n$ is divisible by 3 for $n \neq 4,8$ and $n-b-c=n / 3$.

Suppose first that $\phi(G)$ preserves no symmetric bilinear form. Then $d f_{S}^{H} \geqslant 0$. This can be expressed as

$$
\frac{n(n+1)}{2}-\frac{n(n+2)}{4}-\frac{n(n+3)}{18}-\frac{b(2 n-3 b)}{3}-\sum_{1 \leqslant i \leqslant 3} \frac{m_{i}\left(n-3 m_{i}\right)}{3} \geqslant 0,
$$

whence $(6+24 b) n-n^{2} \leqslant 36\left(b^{2}+m_{1}^{2}+m_{2}^{2}+m_{4}^{2}\right)$. Similarly, $(6+24 c) n-n^{2} \leqslant$ $36\left(c^{2}+m_{3}^{2}+m_{5}^{2}+m_{6}^{2}\right)$. Adding these two inequalities, we get

$$
12 n+18 n^{2} \leqslant 36\left(d_{A}^{\phi(y)}+d_{A}^{\phi(x y)}\right) .
$$


By $T_{A}$ we have $d_{A}^{\phi(y)}+d_{A}^{\phi(x y)} \leqslant n^{2} / 2+2$ so $6 n+9 n^{2} \leqslant 9 n^{2}+36$ which is false for $n>6$.

If $\phi(G)$ is orthogonal then $d f_{S}^{H} \geqslant-2$, so the above computation gives $6 n+9 n^{2} \leqslant$ $9 n^{2}+72$; hence $n \leqslant 12$. As $n$ is divisible by 4 and 3 , we have $n=12$.

Let $p=2$. We use Table B-2. If $n \neq 3$ then from $T_{4}^{2}$ and $T_{5}^{2}$ we get $2 a \leqslant$ $n-m_{0}=n$. As $p=2$, we always have $2 a \geqslant n$, so $n=2 a$. In addition, $n=$ $\left(m_{1}+m_{2}+m_{4}\right)+\left(m_{3}+m_{5}+m_{6}\right)$ implies $m_{1}+m_{2}+m_{4}=n / 2$ and $m_{3}+m_{5}+m_{6}=n / 2$ in view of $T_{4}^{2}$ and $T_{5}^{2}$. By $T_{1}^{2}, T_{2}^{2}$ and $T_{3}^{2}$, we have that $m_{i}+m_{7-i} \leqslant n-b-c$ and by $T_{6}^{2}, T_{7}^{2}$ and $T_{8}^{2}$ that $n-b-c \leqslant m_{i}+m_{7-i}$ for $i=1,2,3$. Hence $3(n-b-c)=n$. The formula for computing $d f_{S}$ makes no difference with that for $p>7$, so the above argument works again and yields that $n \leqslant 12$ and $\phi(G)$ preserves symmetric bilinear form. As $n$ is divisible by $6, n=12$.

Let $p=3$. We use Table B-3. Then $2 a \leqslant n$ by $T_{9}^{3}$ and $n \leqslant 2 a$ by $T_{4}^{3}$ and $T_{5}^{3}$. So $n=2 a$. As above, $n$ is divisible by 4 . In addition, $n=\left(m_{1}+m_{2}+m_{4}\right)+\left(m_{3}+\right.$ $\left.m_{5}+m_{6}\right)$ implies $m_{1}+m_{2}+m_{4}=n / 2$ and $m_{3}+m_{5}+m_{6}=n / 2$ in view of $T_{4}^{3}$ and $T_{5}^{3}$. By $T_{6}^{3}, T_{7}^{3}$ and $T_{8}^{3}$ we have that $n-b-c \leqslant m_{i}+m_{7-i}$ for $i=1,2,3$ and always $n \leqslant 3(n-b-c)$. Hence $3(n-b-c)=n$ and $b+c=2 n / 3$. As $n / 3=n-b-c \geqslant b \geqslant c$ for $p=3$, we have that $b=c=n / 3$. So the Jordan form of $\phi(y)$ is $\left(b J_{3}\right)$ and $3 b=n$. So $n$ is divisible by 12 . Observe that $T_{6}^{3}, T_{7}^{3}$ and $T_{8}^{3}$ are equivalent to $d_{V}^{\phi(y)}=n-b-c \leqslant m_{i}+m_{7-i}$ for $i=1,2,3$. So $m_{i}+m_{7-i} \geqslant n / 3$. As $\sum_{i} m_{i}=n$, we deduce that $m_{i}+m_{7-i}=n / 3$. As $2 d_{S}^{\phi(y)}=d_{A}^{\phi(y)}+b$ by Lemma 4.5 , we have $d_{S}^{\phi(y)}=\left(n^{2}+n\right) / 6$. Therefore,

$d f_{S}^{H}=\frac{n(n+1)}{2}-\frac{n(n+2)}{4}-\frac{n^{2}+n}{6}-\sum_{1 \leqslant i \leqslant 3} m_{i} m_{7-i}=\frac{n^{2}-2 n}{12}-\sum_{1 \leqslant i \leqslant 3} m_{i} m_{7-i}$.

However, $\sum_{1 \leqslant i \leqslant 3} m_{i} m_{7-i}=\sum_{1 \leqslant i \leqslant 3}\left(m_{i}+m_{7-i}\right)^{2} / 2-\left(\sum_{1 \leqslant i \leqslant 6} m_{i}^{2}\right) / 2=n^{2} / 6-$ $d_{A}^{\phi(x y)} / 2$. As $d_{A}^{\phi(x y)}+d_{A}^{\phi(y)}+d_{A}^{\phi(x)} \leqslant n^{2}+2$ and $d_{A}^{\phi(x)}=n^{2} / 2$ and $d_{A}^{\phi(y)}=n^{2} / 3$, we have that $d_{A}^{\phi(x y)} \leqslant n^{2}+2-n^{2} / 2-n^{2} / 3=n^{2} / 6+2$ whence $d_{A}^{\phi(x y)} / 2 \leqslant n^{2} / 12+1$. Therefore, $d f_{S}^{H}=\left(n^{2}-2 n\right) / 12-n^{2} / 6+d_{A}^{\phi(x y)} / 2 \leqslant-n / 6+1$. If $\phi(H)$ preserves a symmetric bilinear form that $-2 \leqslant-n / 6+1$, whence $n \leqslant 18$ so $n=12$. Otherwise, $d f_{S}^{H} \geqslant 0$ and $n \leqslant 6$ which is false.

Proposition 4.10. If $d^{\phi(y)}=2$ (respectively, 1) then $n<12$ (respectively, 8). If $d^{\phi(y)}=2$ and the minimum polynomial of $\phi(x y)$ is of degree 6 then $n<10$.

Proof. Set $X=\phi(x)$ and $Y=\phi(y)$. Observe that $c^{X} \geqslant n^{2} / 2, c^{X Y} \geqslant n^{2} / 7$ (see the proof of Lemma 4.7). By formula (9) $c^{Y} \leqslant 2+n^{2}-c^{X}-c^{X Y} \leqslant 2+5 n^{2} / 14$. As $d^{Y}=2$ (or, respectively, 1), we have $c^{Y} \geqslant 4+(n-2)^{2} / 2$ (respectively, $1+(n-1)^{2} / 2$ ) so $4+(n-2)^{2} / 2 \leqslant 2+5 n^{2} / 14$ (respectively, $1+(n-1)^{2} / 2 \leqslant 2+5 n^{2} / 14$ ). Equivalently, $n^{2}-14 n+28 \leqslant 0$ (respectively, $2 n^{2}-14 n-7 \leqslant 0$ ) whence $n<12$ (respectively, $n<8$ ).

For the additional claim, as the minimum polynomial of $X Y$ is of degree at most 6 , we have $c^{X Y} \geqslant n^{2} / 6$ whence $c^{Y} \leqslant 2+n^{2}-c^{X}-c^{X Y} \leqslant 2+n^{2} / 3$. So $4+(n-2)^{2} / 2 \leqslant 2+n^{2} / 3$, whence $n^{2}-12 n+24 \leqslant 0$. This implies that $n<10$.

Lemma 4.11. Let $\phi: H_{237} \rightarrow \mathrm{GL}(n, F)$ be a rigid representation. Suppose that $\phi(y)$ and $\phi(x y)$ are real. Then $\phi\left(H_{237}\right)$ preserves a symmetric bilinear form and $n \leqslant 8$. In addition, $n \leqslant 6$ for $p=2$, and $n \leqslant 7$ for $p=3$ or 7 . 
Proof. By Lemma 2.12(2), $\phi\left(H_{237}\right)$ preserves a non-degenerate symmetric or alternating bilinear form $f$. Assume $n>2$. By Lemma 2.7, the minimum polynomial of $\phi(y)$ is of degree 3. In particular, $d^{\phi(y)}>0$. As $\phi$ is rigid, $d f_{A}^{H}=-2$. Let $p \neq 3,7$. By Proposition 4.6, $2 d f_{S}^{H}+d^{\phi(y)}+d^{\phi(x y)}=-2$ whence $d f_{S}^{H} \leqslant-2$. By Lemma 2.18, $f$ is symmetric and $d f_{S}^{H}=-2$. Then $d^{\phi(y)}+d^{\phi(x y)}=2$. So $d^{\phi(y)}=1$ or 2 . Let $p \neq 2$. By summing inequalities $T_{17}-T_{22}$ in Table B-1 we get $n \leqslant 6 d^{\phi(y)}+d^{\phi(x y)}$. If $d^{\phi(y)}=1$ then $d^{\phi(x y)}=1$ and $n \leqslant 7$. (Lemma 3.8 gives an example with $d^{\phi(y)}=d^{\phi(x y)}=1$ and $n=7$.) If $d^{\phi(y)}=2$ then $d^{\phi(x y)}=0$ and $n \leqslant 12$. In this case $c^{\phi(x y)} \geqslant n^{2} / 6$ and $c^{\phi(y)}=4+(n-2)^{2} / 2$ whence $n^{2}-12 n+24 \leqslant 0$ by formula (9). This implies $n \leqslant 9$. In fact, $n$ is even as $b=c$ and $d^{\phi(y)}=2$. So $n \leqslant 8$. (If $n=8$, we have an example $[4,4][2,3,3][0,2,1,1,1,1,2]$ in Lemma 3.14(1).)

Let $p=2$. By summing inequalities $T_{1}^{2}, T_{2}^{2}, T_{3}^{2}$ in Table B-2, we get $n \leqslant$ $3 d^{\phi(y)}+d^{\phi(x y)}$. If $d^{\phi(y)}=1$, then $d^{\phi(x y)}=1$ and $n \leqslant 4$. If $d^{\phi(y)}=2$, then $n \leqslant 6$.

Let $p=3$. Let $\operatorname{Jord} \phi(y)=\operatorname{diag}\left(k_{1} J_{1}, k_{2} J_{2}, k_{3} J_{3}\right)$. Then $d^{\phi(y)}=k_{1}+k_{2}+k_{3}$, $b=k_{2}+k_{3}, c=k_{3}$. By Proposition $4.6, k_{1}+k_{3}+d^{\phi(x y)} \leqslant 2$. As the minimum polynomial of $\phi(y)$ is of degree $3, k_{3}>0$. Hence $k_{3}=1$ or 2 . By summing inequalities $T_{11}^{3}-T_{16}^{3}$, we have that $m_{1}+m_{2}+m_{3}+m_{4}+m_{5}+m_{6} \leqslant 6 c=6 k_{3}$ whence $n-m_{0} \leqslant 6 k_{3}$ and $n \leqslant 6 k_{3}+d^{\phi(x y)}$. If $k_{3}=1$ then $k_{1}+d^{\phi(x y)} \leqslant 1$ whence $n \leqslant 7$. (See an example for $n=7$ in Lemma 3.8.) If $k_{3}=2$ then $k_{1}=0$ and $d^{\phi(x y)}=0$ so $n \leqslant 12$. As above we have $n \leqslant 9$. However, $n=2 k_{2}+6$ is even hence $n \leqslant 8$. (There is an example for $n=6$ in Lemma 3.7 for $k_{3}=2$.)

Let $p=7$ and let $\operatorname{Jord} \phi(x y)=\operatorname{diag}\left(k_{1} J_{1}, k_{2} J_{2}, k_{3} J_{3}, k_{4} J_{4}, k_{5} J_{5}, k_{6} J_{6}, k_{7} J_{7}\right)$. Then by Proposition $4.6, k_{1}+k_{3}+k_{5}+k_{7}+d^{\phi(y)} \leqslant 2$. In particular, $d^{\phi(y)} \leqslant 2$. If $d^{\phi(y)}=1$ then $n \leqslant 7$ by Lemma 4.10. (See Lemma 3.8 for an example for $n=7$.) Let $d^{\phi(y)}=2$. Then $k_{1}=k_{3}=k_{5}=k_{7}=0$. In particular, $k_{7}=0$ means that the minimum polynomial of $\phi(x y)$ is of degree at most 6 , hence equal to 6 in view of Lemma 4.7 (provided $n>5$ ). By Lemma 4.10, $n<10$ so $k_{6}=1$ and then $k_{2} \leqslant 1$. If $n>6$ then $k_{2}=1, n=8$ and $\operatorname{Jord} \phi(x y)=\operatorname{diag}\left(J_{2}, J_{6}\right)$. By Lemma 2.27, $f$ is symplectic, hence $d f_{S}^{H} \geqslant 0$.

Proposition 4.12. Let $H=H_{237}$ and $\phi: H \rightarrow \mathrm{GL}(n, F)$ be a rigid representation. Suppose that $\phi(y)$ and $\phi(x y)$ are real. Then one of the following holds:

(1) $n=p=2$ and $\phi(H) \cong \mathrm{SL}(2,8)$;

(2) $n=3, p \neq 2$ and $\phi(H) \cong \operatorname{PSL}(2, \bar{p}) \cong O^{\prime}(3, \bar{p})$;

(3) $n=4$ and $\phi(H) \cong \operatorname{SL}(2, p) \circ \mathrm{SL}(2, p)$ if $p=\bar{p}$ and $\operatorname{PSL}\left(2, p^{3}\right)$ otherwise;

(4) $n=5, p>3$ and $\phi(H) \cong \operatorname{PSL}(2, \bar{p})$;

(5) $n=6, p \neq 2,7$ and $\phi(H) \cong \operatorname{SL}(3,2)$;

(6) $n=7, p \neq 2$ and $\phi(H) \cong \mathrm{SL}(2,8)$;

(7) $n=8, p \neq 2,3,7$ and $\phi(H) \cong \mathrm{SL}(2, p) \circ \mathrm{SL}(2, p)$ if $p=\bar{p}$ and $\operatorname{PSL}\left(2, p^{3}\right)$ otherwise.

Proof. By Lemma 4.11, $n \leqslant 8$. The existence of the representations in (1) - (7) follows from Lemma 3.3 for $n=2$, Lemma 3.5 for $n=3$, Lemma 3.13 for $n=4$, Lemma 3.5 for $n=5$, Lemma 3.7 for $n=6$, Lemma 3.8 for $n=7$ and Lemma 3.14 for $n=8$. In order to show that $\phi$ is one of these representations it suffices to observe, in view of Theorem 2.10, that the multiplicity vector $\left[m^{\phi(x)}\right],\left[m^{\phi(y)}\right],\left[m^{\phi(x y)}\right]$ coincides with a multiplicity vector provided in the above lemmas. This can be easily done by using the determinant conditions and the adjoint test. Let, say, $n=6, p \neq 2$. Then $\left[m^{\phi(x)}\right]=[4,2]$ or $[2,4]$ so the adjoint test combined with the 
determinant conditions implies $\left[m^{\phi(y)}\right]=[2,2,2]$ and $\left[m^{\phi(x y)}\right]=[0,1,1,1,1,1,1]$ if for $p \neq 7$, and $[1,1,1,1,1,1,0]$ for $p=7$. The option $\left[m^{\phi(x)}\right]=[2,4]$ contradicts tests $T_{4}, T_{4}^{3}$ and $T_{1}^{7}$ in Tables B-1, B-3 and B-4, respectively. If $p=7$, the option $\left[m^{\phi(x)}\right]=[4,2]$ contradicts test $T_{0}^{7}$ in Table B-4, otherwise the multiplicity vector in question coincides with that in Lemma 3.7. Let $n=6, p=2$. Then $\left[m^{\phi(x)}\right]$ could be $[4,2]$ or $[3,3]$. At the former case $\left[m^{\phi(y)}\right]=[2,2,2]$ and $\left[m^{\phi(x y)}\right]=[0,1,1,1,1,1,1]$ is the only option which however contradicts test $t_{5}^{2}$ in Table B-2. Let $\left[m^{\phi(x)}\right]=[3,3]$. The option $\left[m^{\phi(y)}\right]=[4,1,1]$ contradicts the adjoint test, so $\left[m^{\phi(y)}\right]=[2,2,2]$ by the determinant condition. As $\phi$ is rigid, $c^{\phi(x y)}=8$ which cannot hold if $\phi(x y)$ is real.

For $n=7,8$ we can argue similarly, but we wish to provide a more conceptual argument. By Lemma 4.11, $p \neq 2,3,7$ if $n=8$ and $p \neq 2$ if $n=7$. Furthermore, we have seen in the first paragraph of the proof of Lemma 4.11 that $\left(d^{\phi(y)}, d^{\phi(x y)}\right)=$ $(1,1)$ if $n=7, p \neq 3$ and $(2,0)$ if $n=8$. If $n=7, p \neq 3$ then $\left[m^{\phi(y)}\right]=[1,3,3]$ hence the only option left by the adjoint test and the determinant condition is $[4,3][1,3,3][1,1,1,1,1,1,1]$ which occurs in Lemma 3.8. If $n=7, p=3$ then $k_{3}=1$ in the proof of Lemma 4.11. It is easy to rule out the option $k_{1}=1$ so $k_{1}=0$ and $m_{0}=1$. The adjoint test and the determinant condition left us with the only option $[4,3][3,3,1][1,1,1,1,1,1,1]$ which occurs in Lemma 3.8. If $n=8$ then $\left[m^{\phi(y)}\right]=[2,3,3]$ and $d^{\phi(x y)}=0$. Then the result follows from Proposition 4.9.

\section{Non-Hurwitz irreducible groups}

In this section we assume that $G \subset \mathrm{GL}(n, F)$ is an irreducible subgroup which preserves no non-zero quadratic form. This is equivalent to saying that $G$ fixes no non-zero element of $S$ or that $d_{S}^{G}=0$ or that $G$ is contained in no orthogonal group.

We start from arbitrary elements $X, Y, Z \in \mathrm{SL}(n, \bar{F}))$ such that $X^{2}=Y^{3}=$ $Z^{7}=\operatorname{Id}$ and $\operatorname{det} X=\operatorname{det} Y=\operatorname{det} Z=1$. (The latter condition is often referred as the determinant condition.) The conjugacy classes of these elements are described by multiplicity vectors of shape $[a, n-a][n-b-c, b, c],\left[m_{0}, m_{1}, m_{2}, m_{3}, m_{4}, m_{5}, m_{6}\right]$ where $m_{0}+\cdots+m_{7}=n$. If $G=\phi\left(H_{237}\right)$ is the image of a representation $\phi$ such that $X=\phi(x), Y=\phi(y)$ and $Z=\phi(x y)$ then the multiplicity vector satisfies conditions $T_{A}, T_{S}$ and $T_{E}$ as well as the conditions in the tables in Appendix B. Our aim is to write down all such vectors. So we arrive at the following algorithm. We look through all multiplicity vectors and discard those which do not satisfy any of the above condition. Vectors we shall be left with are called admissible. This approach makes it convenient to say that a vector passes test $T_{A}$ (or $T_{S}$ etc.) if it satisfies $T_{A}$. Thus, a vector is called admissible if it satisfies the determinant condition and passes all the tests $T_{A}, T_{S}$ and $T_{E}$ as well as those recorded in Appendix B (which consists of Tables B-1, B-2, B-3, B-4, depending on $p$ ). We emphasize that tests $T_{A}, T_{S}$ and $T_{E}$ consist of applying Scott's formula to the adjoint module, symmetric square and exterior square of the representation module $V$, while the tests in the tables of Appendix B are produced by applying Scott's formula to the tensor product of $V$ with the modules constructed in Section 3.

EXAMPLE. Vector $[2,3][3,1,1][1,1,1,0,0,1,1]$ does not satisfy the determinant condition as $\operatorname{det} X=-1$. Vector $[3,2][3,1,1][1,1,1,0,0,1,1]$ does not pass test $T_{0}$ in Table B-1. 
Recall that test $T_{E}$ can be omitted for $p \neq 2$ as every vector passed tests $T_{S}$ and $T_{0}$ passes $T_{E}$. Similarly, if $p=2$ then test $T_{S}$ is not useful. This follows from Proposition 4.6 which describes the dependence between tests $T_{A}, T_{S}, T_{0}$ and $T_{E}$.

The tables in Appendix C-Appendix F list all admissible multiplicity vectors for certain values of $n$ (according to the value of $p$ ). To make the tables shorter we have been forced to omit the vectors which can be obtained from a vector given in a table by the substitution $\omega \rightarrow \omega^{2}$ (that is, up to permuting $b$ and $c$ ) and $\varepsilon \rightarrow \varepsilon^{i}$ (which is equivalent to permuting $m_{1}, m_{2}, m_{3}, m_{4}, m_{5}, m_{6}$ by powers of (132645)). (It would be incorrect to use other permutations.)

The above algorithm has been implemented as a computer program, so the tables in Appendix C-Appendix $\mathrm{F}$ have been obtained as the output of the program. In principle, the necessary computations can be performed manually, as our main results concern matrices of size at most 40 .

Proof of Theorem 1.2. If $n=2$ then $p=2$ and the result follows from Lemma 3.3. For $n=3$ consult [6, Theorem 1]. For $n=4,5,6,7,10$ the result is contained in [6, Theorem 2], except for the case $n=6, p=2$. Let $m^{V}=[a, n-a][n-b-$ $c, b, c]\left[m_{0}, m_{1}, m_{2}, m_{3}, m_{4}, m_{5}, m_{6}\right]$ be the multiplicity vector of $\phi(x), \phi(y), \phi(x y)$. Then it is admissible. For $n<20$ the list of admissible vectors is provided by Tables C-1, D-1, E-1, F-1 (depending on $p$ ). These tables contain no entry for $n=10,11$ which tells us that there is no representation in question. In addition, Table D-1 contains no entry for $n=8,9$, Table E- 1 contains no entry for $n=13,14$ and Table F-1 contains no entry for $n=12,17,18$ which leads to the similar conclusion for $p=2, n=8,9 ; p=3, n=13,14 ;$ and $p=7, n=8,9,12,17,18$.

If $p=2$ then the entries in Table D- 1 for $n=6,13$ are of rigidity index 0 . If $p \neq 2,3$ then for $n=8,9$ and 13 the entries in Tables C-1 and F-1 are of rigidity index 0 which tells us that $\phi$ is rigid if it exists. The existence of $\phi$ for all the cases is proved in Section 3. So for these cases the theorem follows from the results of Section 3.

REMARK. We do not identify rigid representations of dimension 14 so the question of their existence remains open.

Proposition 5.1. The group $G=\operatorname{PSp}(6,3)$ is not Hurwitz.

Proof. By [1], $G$ has a complex irreducible representation of dimension 13 whose image preserves no bilinear form. By Theorem 1.2, $G$ is not Hurwitz.

Proposition 5.2. The groups $G=\operatorname{Sp}(6, q)$ with $q$ even are not Hurwitz.

Proof. Suppose the contrary. Let $\phi: H_{237} \rightarrow \mathrm{Sp}(6, q)$ be a surjective homomorphism and set $X=\phi(x), Y=\phi(y)$. Let $V$ be the natural module for $\operatorname{Sp}(6, q)$. By Lemma $2.7 d^{Y}>0$. So $d^{X} \geqslant 3, d^{Y} \neq 1,3$ (as $Y$ is real). By formula (5) $d^{Y}<4$. So $d^{Y}=2$, and hence $d^{X Y} \leqslant 1$ by (5). As $X Y$ is real, $d^{X Y}=0$. As $c^{X}+c^{Y}+c^{X Y} \leqslant 38$, $c^{Y}=12$ and $c^{X} \geqslant 18$, we have that $c^{X Y} \leqslant 8$. If some eigenvalue $\varepsilon \neq 1$ of $X Y$ is of multiplicity 2 then $c^{X Y} \geqslant 10$. Hence $c^{X Y}=6$ so each eigenvalue is of multiplicity 1 and the multiplicity vector of $X Y$ is $[0,1,1,1,1,1,1]$. By formula $T_{4}^{2}$ in Table B-2, $d^{X}=3$. So the multiplicity vector of $X, Y, X Y$ is $[3,3][2,2,2][0,1,1,1,1,1,1]$.

Let $\bar{F}_{2}$ be an algebraically closed field of characteristic 2 . We first observe that $\operatorname{Sp}\left(6, \bar{F}_{2}\right)$ contains a unique conjugacy class of elements of order 3 and 7 with the 
above multiplicity vector. By Witt's theorem it suffices to show that there are bases of $\bar{F}_{2}^{6}$ with Gram matrix $\left(\begin{array}{cc}0 & \text { Id } \\ \text { Id } & 0\end{array}\right)$ such that $Y$ makes shape $\operatorname{diag}\left(\omega, \omega, 1,1, \omega^{2}, \omega^{2}\right)$, and $X Y$ makes shape $\left.\operatorname{diag}\left(\varepsilon, \varepsilon^{2}, \varepsilon^{3}, \varepsilon^{4}, \varepsilon^{5}, \varepsilon^{6}\right)\right)$. Indeed, it is easy to observe that every element $g \in \operatorname{Sp}\left(6, \bar{F}_{2}\right)$ of odd order preserves a totally isotropic subspace $W$ of dimension 3. Moreover, $W$ can be chosen so that under certain basis $b_{1}, b_{2}, b_{3}$ of $W$ the matrix of $\left.Y\right|_{W}$ would be $\operatorname{diag}(\omega, \omega, 1)$ and $\operatorname{diag}\left(\varepsilon, \varepsilon^{2}, \varepsilon^{3}\right)$ for $\left.X Y\right|_{W}$. It is well known that $b_{1}, b_{2}, b_{3}$ can be complemented to a basis of $\bar{F}_{2}^{6}$ (called a Witt basis) with the above Gram matrix. This justifies the claim.

By [1] (see the character table of $\operatorname{Sp}(6,2)$ ), the conjugacy classes of elements in classes $3 C$ and $7 A$ have the above multiplicity vectors. It follows that $Y$ and $X Y$ are conjugate in $\operatorname{Sp}(6, F)$ to elements of $\operatorname{Sp}(6,2)$ from classes $3 C$ and $7 A$.

By Steinberg's theorem [18, Theorem 49], every irreducible $\bar{F}_{2}$-representation of $\operatorname{Sp}(6,2)$ extends to a representation of $\operatorname{Sp}\left(6, \bar{F}_{2}\right)$ and hence of $\operatorname{Sp}(6, q)$. In particular, as $\operatorname{Sp}(6,2)$ has an irreducible representation of degree 8 (see [2]), this also true for $\operatorname{Sp}\left(6, \bar{F}_{2}\right)$ and $\operatorname{Sp}(6, q)$. Denote the representation of $\operatorname{Sp}(6, q)$ of degree 8 by $\tau$. The trace of $\tau(Y)$ equals 2 and the trace of $\tau(X Y)$ equals 1 as is for the restriction of $\tau$ to $\operatorname{Sp}(6,2)$; see [2]. Therefore, $d^{\tau(Y)}=4$ and $d^{\tau(X Y)}=2$. As $d^{\tau(X)} \geqslant 4$, this contradicts formula (5).

The results of the previous sections are valid for almost arbitrary $q$. Here we consider more special cases.

Definition 5.3. An element $g \in \mathrm{GL}(n, F)$ of order 7 (and its conjugacy class) is called

$\left\{\begin{array}{l}\text { rational, if } p \neq 7 \text { and } g \text { is conjugate in } \operatorname{GL}(n, F) \text { to } g^{i} \text { for } 1 \leqslant i \leqslant 6 \\ \text { semirational, if } p \neq 7 \text { and } g \text { is conjugate in } \operatorname{GL}(n, F) \text { to } g^{2} .\end{array}\right.$

If $g \in \mathrm{GL}(n, F)$ is unipotent, its conjugacy class always meets $\operatorname{GL}(n, q)$ and $U(n, q)$. The condition for $\operatorname{Sp}(n, q)$ and $O(n, q)$ is recorded in Lemma 2.27. If $g$ is semisimple then the similarity class of $g$ does not always meet $G$. This depends on certain conditions on $q$ which can be described in terms of symmetries of the eigenvalue multiplicities or, equivalently, in terms of symmetries of the multiplicity vector of $g$. We only need to state the conditions for $g$ of order 3 or 7 . To do this, we introduce a function $s(g)$ called the symmetry type of $g$. If $|g|=3$, we define $s(g)=2$ if $g$ is real and 1 otherwise. Let $|g|=7$. We set $s(g)=6$ if $g$ is rational, otherwise $s(g)=3,2,1$ if $g$ is, respectively, semirational, real or neither of these.

In order to tabulate the information let $g, h \in \mathrm{GL}(n, F)$ and $|g|=3,|h|=7$. Table 3 (which is a rearrangement of [6, Table 5]) indicates conditions on $s(g), s(h)$ which guarantee that the similarity classes of $g$ and $h$ meet $G$. We use $*$ to express the absence of any condition; for example, $(*, 6)$ means that $h$ is rational, and $s(g)$ may be 1 or 2 . Observe that if $[n-b-c, b, c]$ and $\left[m_{0}, m_{1}, m_{2}, m_{3}, m_{4}, m_{5}, m_{6}\right]$ are the multiplicity vectors of $g, h$, respectively, then the symmetry type of $(g, h)$ is expressed in terms of these vectors as follows. We have $s(g)=2$ if and only if $b=c$. We have $s(h)=6$ if and only if $m_{1}=\cdots=m_{6} ; s(h)=3$ if and only if $m_{1}=m_{2}=m_{4} \neq m_{3}=m_{5}=m_{6} ; s(h)=2$ if and only if $m_{i}=m_{7-i}(i=1,2,3)$ but not all $m_{i}$ coincide. In the column headed by $\operatorname{Sp}(n, q)$ the entry with - refers to Lemma 2.27. 
Lemma 5.4. Let $1 \neq g, h \in G$ where $G \in\{\operatorname{GL}(n, q), U(n, q), \operatorname{Sp}(n, q)\}$. Suppose that $g^{3}=1$ and $h^{7}=1$. Then $(s(g), s(h))$ takes one of the values indicated in Table 3 .

Observe that $2^{6 k} \equiv 1(\bmod 21), 2^{6 k+1} \equiv 2(\bmod 21), 2^{6 k+2} \equiv 4(\bmod 21), 2^{6 k+3} \equiv$ $8(\bmod 21), 2^{6 k-2} \equiv-5(\bmod 21)$ and $2^{6 k-1} \equiv-10(\bmod 21)$. Therefore, if $q$ is even then one has to use only 1 st, 3 rd, 5 th and 7 th rows of Table 3.

Table 3: Symmetry types of elements of order 3 and 7 in classical groups.

\begin{tabular}{|l|l|l|l|}
\hline \multicolumn{1}{|c|}{$q$} & \multicolumn{1}{|c|}{$\mathrm{GL}(n, q)$} & \multicolumn{1}{|c|}{$U(n, q)$} & \multicolumn{1}{|c|}{$\operatorname{Sp}(n, q)$ and } \\
& & & \\
\hline$q \equiv 1(\bmod 21)$ & $(*, *)$ & $(2,2)$ or $(2,6)$ & $(2,2)$ or $(2,6)$ \\
\hline$q \equiv-1(\bmod 21)$ & $(2,2)$ or $(2,6)$ & $(*, *)$ & $(2,2)$ or $(2,6)$ \\
\hline$q \equiv 2,-10(\bmod 21)$ & $(2,3)$ or $(2,6)$ & $(*, 6)$ & $(2,6)$ \\
\hline$q \equiv-2,10(\bmod 21)$ & $(*, 6)$ & $(2,3)$ or $(2,6)$ & $(2,6)$ \\
\hline$q \equiv 4,-5(\bmod 21)$ & $(*, 3)$ or $(*, 6)$ & $(2,6)$ & $(2,6)$ \\
\hline$q \equiv-4,5(\bmod 21)$ & $(2,6)$ & $(*, 3)$ or $(*, 6)$ & $(2,6)$ \\
\hline$q \equiv 8(\bmod 21)$ & $(2, *)$ & $(*, 2)$ or $(*, 6)$ & $(2,2)$ or $(2,6)$ \\
\hline$q \equiv-8(\bmod 21)$ & $(*, 2)$ or $(*, 6)$ & $(2, *)$ & $(2,2)$ or $(2,6)$ \\
\hline$q=3^{6 k}$ & $(*, *)$ & $(*, 2)$ or $(*, 6)$ & $(-, 2)$ or $(-, 6)$ \\
\hline$q=3^{6 k+3}$ & $(*, 2)$ or $(*, 6)$ & $(*, *)$ & $(-, 2)$ or $(-, 6)$ \\
\hline$q=3^{6 k \pm 1}$ & $(*, 6)$ & $(*, 3)$ or $(*, 6)$ & $(-, 6)$ \\
\hline$q=3^{6 k \pm 2}$ & $(*, 3)$ or $(*, 6)$ & $(*, 6)$ & $(-, 6)$ \\
\hline$q \equiv 0(\bmod 7)$ & $(*, *)$ & $(2, *)$ & $(2,-)$ \\
\hline
\end{tabular}

Let $p \neq 2,3,7$. A vector $[a, n-a][n-b-c, b, c]\left[m_{0}, m_{1}, m_{2}, m_{3}, m_{4}, m_{5}, m_{6}\right]$ is called admissible if it passes all tests $T_{0}-T_{30}$ of Table B-1 and tests $T_{A}$ and $T_{S}$. In order to take account of the symmetry type, we introduce the following notation. For $s \in\{1,2\}$, and $t \in\{1,2,3,6\}$ we denote by $N(s, t)$ the set of all natural numbers $n$ such that there is no admissible multiplicity vector which symmetry type is $(k s, l t)$ for some integers $k, l$. For instance, $n=12$ belongs to $N(2,2), N(2,3), N(2,6)$, $N(1,6), N(1,3)$ and does not belong to $N(1,1)$ and $N(2,1)$ as for $n=12$ the admissible vectors are of symmetry type $(2,1)$; see Table C-1. Similarly, the entries for $n=15$ are of symmetry type $(1,6)$ or $(2,1)$ so 15 belongs to $N(2,6), N(2,2)$ and $N(2,3)$ and does not belong to $N(1,1), N(2,1), N(1,3)$ and $N(1,6)$. Observe that $N(s, t) \subseteq N(2,6)$. If $p=2$ we denote a similar set by $N_{2}(s, t)$. If $p=3$ or 7 , we use notation $N_{3}(*, t)$ or $N_{7}(s, *)$ for a similar purpose. (We did not define the notion of similarity type for unipotent elements. So $n \in N_{3}(*, t)$ means that there is no admissible multiplicity vector which symmetry type is $(*, l t)$ for some integer $l$.) 
In fact, the main use of the tables in Appendix $\mathrm{C}$-Appendix $\mathrm{F}$ is for deducing the following lemma.

Lemma 5.5. Assume $12 \leqslant n \leqslant 40$ and $n \neq 13$.

(1) Let $n \in N(2,6)$. Then $n \in\{12,14,15,16,17,18,19,22,23,24,25,31\}$.

(2) Let $n \in N(1,6)$. Then $n \in\{12,16,17,18,23,24\}$.

(3) Let $n \in N(2,3)$. Then $n \leqslant 19$ or $n=23$.

(4) Let $n \in N(1,3)$. Then $n \in\{12,23\}$.

(5) Let $n \in N(2,2)$. Then $n \leqslant 19$ or $n=22$.

(6) Let $n \in N(1,2)$. Then $n \in\{12,16,17,18\}$.

(7) Let $n \in N(2,1)$. Then $n=14$.

(8) Let $n \in N_{2}(2,6)$. Then $n<21$ or $n \in\{22,23,24,25,26,30,31,32,38\}$.

(9) Let $n \in N_{2}(1,6)$. Then $n \in\{12,16,17,18,19,22,23,24,25,31\}$.

(10) Let $n \in N_{2}(2,3)$. Then $n<18$ or $n \in\{19,22,23,24,25\}$.

(11) Let $n \in N_{2}(1,3)$. Then $n \in\{12,16,17,19,22,23\}$.

(12) Let $n \in N_{2}(2,2)$. Then $n \leqslant 20$ or $n=22,23$.

(13) Let $n \in N_{2}(2,1)$. Then $n=14$.

(14) Let $n \in N_{2}(1,2)$. Then $n=12,16,17,18$.

(15) Let $n \in N_{3}(*, 6)$. Then $n<20$ or $n \in\{22,23,24,25,31\}$.

(16) Let $n \in N_{3}(*, 3)$. Then $n<17$ or $n \in\{18,19,22,23,25\}$.

(17) Let $n \in N_{3}(*, 2)$. Then $n<20$ or $n \in\{22,31\}$.

(18) Let $n \in N_{7}(2, *)$. Then $n<20$ or $n=22$.

Proof. This is achieved by inspection of the tables in Appendix C-Appendix F.

REMARK. The restriction $n \leqslant 40$ in Lemma 5.5 is sufficient in order to prove our results. However, we could show that $N(2,6)$ hence $N(s, t)$ contains no entries for $n>40$.

In order to make transparent the matter of significance of Lemma 5.5 for determining non-Hurwitz groups, we record the following statement.

LEMMA 5.6. Let $\phi: H_{237} \rightarrow \mathrm{SL}(n, q)$ (respectively, $H_{237} \rightarrow \mathrm{SU}(n, q)$ ) be an absolutely irreducible representation. Suppose that $\phi(H)$ preserves no non-zero symmetric bilinear form. Then $n \notin N(s, t)$ for $N(s, t)$ positioned in the row with the above $q$ in the second (respectively, third) column of Table 4. In particular, $\mathrm{SL}(n, q)$ (respectively, $\mathrm{SU}(n, q)$ ) is not Hurwitz if $n \in N(s, t)$.

Proof. The multiplicity vector of $\phi(x), \phi(y), \phi(x y)$ is obviously admissible. As $q$ is given, its symmetry type $(s, t)$, say, should agree with Table 3 . So $n \notin N(s, t)$ by the definition of $N(s, t)$.

Proof of Theorem 1.3. By Lemma 5.6, we only have to determine the sets $N(s, t)$, $N_{2}(s, t), N_{3}(*, t)$ and $N_{7}(s, *)$ to fill the appropriate boxes in Tables 1 and 2. This has been done in Lemma 5.5.

Corollary 5.7. (1) The group $\mathrm{SL}(n, 2)$ is not Hurwitz for $n<18$ and $n \in$ $\{19,22,23,24,25\}$.

(2) $\operatorname{SL}(n, 3)$ is not Hurwitz for $n<20$ and $n \in\{22,23,24,25,31\}$. 
Table 4: Symmetry types and admissible multiplicity vectors.

\begin{tabular}{|c|c|c|}
\hline & $\mathrm{SL}(n, q)$ & $\mathrm{SU}(n, q)$ \\
\hline$q \equiv 1(\bmod 21)$ odd & $N(1,1)$ & $N(2,2)$ \\
\hline$q \equiv-1(\bmod 21)$ odd & $N(2,2)$ & $N(1,1)$ \\
\hline$q \equiv 2,-10(\bmod 21)$ odd & $N(2,3)$ & $N(1,6)$ \\
\hline$q \equiv-2,10(\bmod 21)$ odd & $N(1,6)$ & $N(2,3)$ \\
\hline$q \equiv 4,-5(\bmod 21)$ odd & $N(1,3)$ & $N(2,6)$ \\
\hline$q \equiv-4,5(\bmod 21)$ odd & $N(2,6)$ & $N(1,3)$ \\
\hline$q \equiv 8(\bmod 21)$ odd & $N(2,1)$ & $N(1,2)$ \\
\hline$q \equiv-8(\bmod 21)$ odd & $N(1,2)$ & $N(2,1)$ \\
\hline$q=3^{6 k}$ & $N_{3}(*, 1)$ & $N_{3}(*, 2)$ \\
\hline$q=3^{6 k+3}$ & $N_{3}(*, 2)$ & $N(*, 1)$ \\
\hline$q=3^{6 k \pm 1}$ & $N_{3}(*, 6)$ & $N_{3}(*, 3)$ \\
\hline$q=3^{6 k \pm 2}$ & $N_{3}(*, 3)$ & $N_{3}(*, 6)$ \\
\hline$q \equiv 0(\bmod 7)$ & $N_{7}(1, *)$ & $N_{7}(2, *)$ \\
\hline$q=2^{6 k} \equiv 1(\bmod 21)$ & $N_{2}(1,1)$ & $N_{2}(2,2)$ \\
\hline$q=2^{6 k \pm 1} \equiv 2,-10(\bmod 21)$ & $N_{2}(2,3)$ & $N_{2}(1,6)$ \\
\hline$q=2^{6 k \pm 2} \equiv 4,-5(\bmod 21)$ & $N_{2}(1,3)$ & $N_{2}(2,6)$ \\
\hline$q=2^{6 k+3} \equiv 8(\bmod 21)$ & $N_{2}(2,1)$ & $N_{2}(1,2)$ \\
\hline
\end{tabular}

Proof of Theorem 1.4. Set $G=\phi(H)$. Let $p \neq 2,3,7$. If $q \not \equiv \pm 1(\bmod 7)$ then the symmetry type of Hurwitz generators for $G$ is $(2,6)$, otherwise $(2,2)$ or $(2,6)$; see Table 3. Therefore, $n \notin N(2,6)$ in the former case and $n \notin N(2,2)$ in the latter case. As $n$ is even, this coincides with what is recorded in statement (1) of the theorem. Let $p=3$. Then $n \notin N_{3}(*, 2)$ if $q=3^{3 l}$ (which is a power of 27) otherwise $n \notin N_{3}(*, 6)$. Let $p=7$. Then $n \notin N_{7}(2, *)$. Of course, in all the cases we have to choose even $n$. This implies the theorem for odd $p$. 
Let $p=2$. The case $n=6$ has been settled in Proposition 5.2. Part (3) is contained in Theorem 2.28 which also contains (2) for $n=10$. Suppose that $q \not \equiv$ $1(\bmod 7)$ or, equivalently, $q=2^{6 k \pm 1}$ or $q=2^{6 k \pm 2}$. Then the multiplicity vector in question must be of $(2,6)$ symmetry. Inspection of Table D-4 shows that $n \neq$ 18,24 unless $G$ preserves a quadratic form. (Theorem 2.28 follows from Table D-4 as well.) In addition, we show that $\phi$ cannot exist if $n=12$ or 16 . Let $m^{V}$ be the multiplicity vector of $\phi(x), \phi(y), \phi(x y)$. Let $n=12$. Suppose first that $m^{V}=$ $[6,6][4,4,4][0,2,2,2,2,2,2]$. Then $d f_{A}=0,2 d f_{S}=d f_{A}-d^{y}-d^{z}=-4, d f_{S}=-2$, $d f_{V}=2, d f_{E}=-4$ which contradicts Lemma 2.18. The same holds if $[6,6]$ is replaced by $[7,5]$. For other choices of $m^{V}$ we have that $d f_{A}<-2$. Let $n=16$. Suppose first that $m^{V}=[8,8][4,6,6][4,2,2,2,2,2,2]$. Then $d f_{A}=256-128-16-$ $72-16-24=0,2 d f_{S}=d f_{A}-d^{y}-d^{z}=-8, d f_{S}=-4$, which is false. The option $m^{V}=[8,8][6,5,5][4,2,2,2,2,2,2]$ contradicts $T_{0}^{2}$.

REMARK. One can expect that Lemma 2.27(3) is useful to improve these results. Indeed, one can observe that a few entries of symmetry $(2,2)$ and $(2,6)$ in Appendix E and Appendix F do not satisfy Lemma 2.27(3). However, this does not affect the final list of $n$ in Theorem 1.4.

Proof of Theorem 1.5. Table G-1 contains no entry with $n=10$. If $p=3$ then Table G-3 contains no entry with $n=8,10,11,14,17$. If $p=7$ then Table G-5 contains no entry with $n=9,10,11,13,16,18$. So for these values of $n$ the result follows.

Proof of Corollary 1.6. Suppose the contrary. Let first $q=7^{k}$. By Theorem 1.4, $\operatorname{Sp}(8, q)$ is not Hurwitz (Theorem 1.4), so there is a surjective homomorphism $\tilde{H} \rightarrow \operatorname{Sp}(8, q)$ where $\tilde{H}$ is a 2 -fold covering of $H_{237}$ (see Section 3 ). This leads to an irreducible representation $\theta: \tilde{H} \rightarrow \operatorname{Sp}(8, F)$ such that $\theta(\tilde{H}) \cong \operatorname{Sp}(8, q)$ (where $F$ is an algebraically closed field of characteristic 7$)$. Let $\phi: \tilde{H} \rightarrow \operatorname{GL}(2, F)$ be the representation described in Lemma 3.3(3). Then $\phi \otimes \theta$ is an irreducible representation of dimension 16 . As $Z(\tilde{H})$ belongs to the kernel of $\phi \otimes \theta$, this can be viewed as a representation of $H_{237}$. It is irreducible and $(\phi \otimes \theta)(\tilde{H})$ is isomorphic to a central product $\operatorname{Sp}(8, q) \circ \mathrm{SL}(2,7)$. Observe that $\theta(\tilde{H})$ and $\phi(\tilde{H})$ preserve bilinear forms with skew symmetric Gram matrices $A, B$, say. Hence $(\phi \otimes \theta)(\tilde{H})$ preserves a bilinear form with matrix $A \otimes B$ which is symmetric. This contradicts Theorem 1.5 .

Let $q=3^{k}$. By Theorem 1.5 groups $\Omega^{ \pm}(8, q)$ are not Hurwitz. As $\Omega^{-}(8, q)$ is centerless, we are left with examining the case where there is a surjective representation $\theta: \tilde{H} \rightarrow \Omega^{+}(8, q) \subset O(8, F)$. Let $\phi: \tilde{H} \rightarrow \mathrm{SL}(2, F)$ and $\tau=\phi \otimes \theta$. As in the previous paragraph, $\tau(\tilde{H})$ preserves a skew symmetric bilinear form hence $\tau(\tilde{H})$ is contained in $\operatorname{Sp}(16, F)$. As $\tau\left(\tilde{x}^{2}\right)=\mathrm{Id}$, one can view $\tau$ as a representation of $H$, which contradicts Theorem 1.4.

Let $n=10$. Let $G$ denote $G^{+}=\Omega^{+}(10, q)$ or $G^{-}=\Omega^{-}(10, q)$. If $q$ is even then the result is contained in Theorem 1.4(2). Let $q$ be odd. If - Id $\notin G$ then the result follows from Theorem 1.5. So assume that $-\mathrm{Id} \in G$. Suppose that $G / Z(G)$ is Hurwitz. Then there is a surjective homomorphism $\theta: \tilde{H} \rightarrow G$. Let $\phi: \tilde{H} \rightarrow$ $\mathrm{SL}(2, F)$ (as above) and $\tau=\phi \otimes \theta$. Then $\tau(\tilde{H})$ preserves a skew symmetric bilinear 
form hence $\tau(\tilde{H})$ is contained in $\operatorname{Sp}(20, F)$. As above, this contradicts Theorem 1.4(1). (Observe that $-\mathrm{Id} \in G^{+}$if $q \equiv 1(\bmod 4)$, otherwise - Id $\in G^{-}$; see $[\mathbf{1 1}$, Proposition 2.5.13].)

Remark. For $p=7$, Corollary 1.6 can be proved straightforwardly. For this one has to observe that the Jordan normal form of $\theta(x y)$ does not have block $J_{7}$ by Lemma 2.27. Computing $d f_{A}$ and $d f_{E}$ one gets a contradiction.

Proof of Theorem 1.7. Suppose first that $p \neq 3,7$. Then the symmetry type of the multiplicity vector in question is $(2,6)$. Tables G-1, G-2 contain no entry of this symmetry for $n=9,11,17,18,24$. So the result follows. Let $p=3$. Then the symmetry type is $(*, 6)$. Tables $\mathrm{G}-3$ and $\mathrm{G}-4$ contain no entry with $(*, 6)$ symmetry for $n=8,9,10,11,16,17,18,23,24$. This yields the result for $p=3$. (The values $n=8,11,17$ have been excluded from the statement as they have already occurred in Theorem 1.5.)

Proof of Theorem 1.8. Suppose the contrary. Then $\operatorname{SL}(n, 2)$ and $\operatorname{SL}(n, 3)$ are Hurwitz. By Corollary 5.7, at least one of these groups is not Hurwitz for $n<20$, $22 \leqslant n \leqslant 26$ and $n=30,31,32$. To treat the other cases let $X, Y \in G$ be such that $X^{2}=Y^{3}=(X Y)^{7}=$ Id. Let $m$ be the multiplicity vector for the triple $X, Y, X Y$. Then $m$ is of $(2,6)$ symmetry type, and similarly for $X(\bmod p), Y(\bmod p)$ and $X Y(\bmod p)$ for $p=2$. If $p=3,7$ then the symmetry types are $(*, 6)$ and $(2, *)$, respectively. This tells us that $\mathrm{SL}(n, 2)$ and $\mathrm{SL}(n, 3)$ have $(2,3,7)$-generators of $(2,6)$ and $(*, 6)$, respectively. Therefore, the values $n=20,26,38$ can be discarded as the rows of Tables D-2 and D-3 for $n=20,26$ and 38 contain no vector of $(2,6)$ symmetry.

Let $n=29$. According to Table C-2, there are exactly 2 options for multiplicity vectors of $(2,6)$-symmetry type, namely, $[13,16][9,10,10][5,4,4,4,4,4,4]$ and $[15,14][9,10,10][5,4,4,4,4,4,4]$. The second option has to be discarded as the rows of Table E- 2 for $n=29$ contain no vector of $(*, 6)$ symmetry and with the $X$-entry $[15,14]$. Consider the option with $X$-entry $[13,16]$. Then $X$ is conjugate in $\operatorname{GL}(n, \mathbf{Z})$ to a matrix of shape $\operatorname{diag}(-1, \ldots,-1,1, \ldots, 1, M, \ldots, M)$ where $M=\left(\begin{array}{ll}0 & 1 \\ 1 & 0\end{array}\right)$ and $M, 1$ or -1 may not occur. Let $r, s, t$ be the number of occurrences of $M,-1$ and 1, respectively. Then the multiplicity vector of $X$ is $[r+t, r+s]$. Obviously, Jord $X(\bmod 2)$ is $\operatorname{diag}\left(r J_{2},(s+t) J_{1}\right)$, and the multiplicity vector of this matrix is $[r+s+t, r]$. If $[r+t, r+s]=[13,16]$ then $r+s+t \geqslant 16$. Hence the multiplicity vector of $X(\bmod 2)$ cannot be $[15,14]$, which is the only option allowed by Table D-2.

Let $n=37$. Table C-3 gives us the following options for the multiplicity vector, namely, $\quad[17,20][11,13,13][7,5,5,5,5,5,5],[17,20][13,12,12][7,5,5,5,5,5,5]$ and $[19,18][11,13,13][7,5,5,5,5,5,5]$. As $[19,18][11,13,13][7,5,5,5,5,5,5]$ is the only vector of symmetry $(2,6)$ for $n=37$ in Table D-3, we immediately dispose of the second option, while the first option can be ruled out as was done for $n=29$. Indeed, $r+s+t \geqslant 20$ so the multiplicity vector of $X(\bmod 2)$ cannot be $[19,18]$. So we are left with the third option. However, the multiplicity vector of $X(\bmod 3)$ cannot be $[19,18]$ by Table E- 4 . 


\section{Tables}

Here we explain how to read the tables given below.

In Appendix A, Tables A-1 to A-4 list multiplicity vectors for rigid representations described in Section 3. It is partitioned into Tables A-1 $(p \neq 2,3,7)$, A-2 $(p=2), \mathrm{A}-3(p=3)$ and A-4 $(p=7)$. Recall that $\bar{p}=p$ if $p\left(p^{2}-1\right)$ is divisible by 7 ; otherwise $\bar{p}=p^{3}$.

Tables B-1 to B-4 of Appendix B list formulas to be satisfied by the multiplicity vectors of irreducible representations of $H_{237}$. The restriction on $n$ in the $3 \mathrm{rd}$ column is for the reader's guidance only. In fact, the right restriction is weaker, and can be extracted from the lemma indicated in the 'reference' column.

The tables in Appendix $\mathrm{C}$-Appendix $\mathrm{F}$ list admissible multiplicity vectors of certain dimensions, that is, those which pass the tests $T_{A}, T_{S}, T_{E}$ and the tests from Tables B. (Test $T_{E}$ is used only for $p=2$; see the remark after Proposition 4.6.) In all these tables except D-4 passing $T_{S}$ means that $d f_{S} \geqslant 0$ and passing $T_{E}$ means that $d f_{E} \geqslant 0$. So the tables are used for showing that $H_{237}$ does not have irreducible representations with certain multiplicity vectors preserving no symmetric bilinear form. (See Proposition 2.18 and the comments following it.) In contrast, Table D-4 has been created assuming that test $T_{S}$ means $d f_{S} \geqslant-1$ and $T_{E}$ means $d f_{E} \geqslant-2$. In addition, we require each multiplicity vector to be of $(2,2)$ or $(2,6)$ symmetry according with Table 3 . Thus, in this case we call a multiplicity vector admissible 'symplectic' if it is of $(2,2)$ or $(2,6)$ symmetry and passes tests $T_{0}^{2}-T_{16}^{2}$ of Table B-2 and tests $T_{A}, T_{S}, T_{E}$. Table D-4 is used for showing that $H_{237}$ does not have irreducible representations with certain multiplicity vectors in characteristic 2 preserving a symmetric bilinear form and no alternating bilinear form. Observe that Lemma 2.27 has not been used for producing Table D-4; however, every entry of this table satisfies Lemma 2.27.

In Appendix G, Tables G-1 to G-5 list admissible 'orthogonal' multiplicity vectors. If $p \neq 2,3,7$ then a multiplicity vector

$$
m_{V}=\left[m_{V}^{\phi(x)}\right]\left[m_{V}^{\phi(y)}\right]\left[m_{V}^{\phi(x y)}\right]
$$

is called admissible 'orthogonal' if it is of $(2,2)$ or $(2,6)$ symmetry, passes tests $T_{A}$, $T_{0}-T_{30}$ and $d f_{S} \geqslant-2$. If $p=3$ (respectively, 7 ) then a multiplicity vector is called admissible orthogonal if it is of $(*, 2)$ (respectively, $(2, *))$ symmetry, passes tests $T_{A}, T_{0}^{3}-T_{22}^{3}$ (respectively, $T_{0}^{7}-T_{5}^{3}$ ) and $d f_{S} \geqslant-2$ and, additionally, the respective Jordan form of $\phi(y)$ (respectively, $\phi(x y)$ satisfies the condition of Lemma 2.27.

We observe that the tables in Appendix A in fact contain all rigid irreducible representations in dimension less than 14. Indeed, if both $\phi(y), \phi(x y)$ are real, this follows from Proposition 4.12. If $\phi(y)$ or $\phi(x y)$ is not real then $\phi\left(H_{237}\right)$ preserves no symmetric bilinear form (Lemma 2.27). Therefore, the multiplicity vector of $\phi$ is admissible. This means that the multiplicity vector of $\phi$ appears in the tables in Appendix C-Appendix F. By inspection of these tables, there are no other admissible multiplicity vector for $n<14$ except those in the tables of Appendix A. 
Appendix: the tables

Appendix A. Multiplicity vectors for rigid representations of $H_{237}$

Table A-1: $1<n \leqslant 13$ and $p \neq 2,3,7$.

\begin{tabular}{|c|c|c|c|c|}
\hline no. & $\operatorname{dim}$ & multiplicity vector & $p$ & image \\
\hline 1 & 3 & {$[1,2][1,1,1][1,1,0,0,0,0,1]$} & & $\mathrm{PSL}_{2}(\bar{p})$ \\
\hline 2 & 3 & {$[1,2][1,1,1][1,0,1,0,0,1,0]$} & & $\mathrm{PSL}_{2}(\bar{p})$ \\
\hline 3 & 3 & {$[1,2][1,1,1][1,0,0,1,1,0,0]$} & & $\mathrm{PSL}_{2}(\bar{p})$ \\
\hline 4 & 3 & {$[1,2][1,1,1][0,1,1,0,1,0,0]$} & & $\mathrm{SL}(3,2)$ \\
\hline 5 & 3 & {$[1,2][1,1,1][0,0,0,1,0,1,1]$} & & $\mathrm{SL}(3,2)$ \\
\hline 6 & 4 & {$[2,2][2,1,1][0,1,1,0,0,1,1]$} & $\left(7, p^{2}-1\right)=1$ & $\mathrm{PSL}_{2}\left(p^{3}\right)$ \\
\hline $6^{\prime}$ & 4 & {$[2,2][2,1,1][0,1,1,0,0,1,1]$} & $\left(7, p^{2}-1\right) \neq 1$ & $\mathrm{SL}_{2}(p) \circ \mathrm{SL}_{2}(p)$ \\
\hline 7 & 4 & {$[2,2][2,1,1][0,1,0,1,1,0,1]$} & $\left(7, p^{2}-1\right)=1$ & $\operatorname{PSL}_{2}\left(p^{3}\right)$ \\
\hline $7^{\prime}$ & 4 & {$[2,2][2,1,1][0,1,0,1,1,0,1]$} & $\left(7, p^{2}-1\right) \neq 1$ & $\mathrm{SL}_{2}(p) \circ \mathrm{SL}_{2}(p)$ \\
\hline 8 & 4 & {$[2,2][2,1,1][0,0,1,1,1,1,0]$} & $\left(7, p^{2}-1\right)=1$ & $\mathrm{PSL}_{2}\left(p^{3}\right)$ \\
\hline $8^{\prime}$ & 4 & {$[2,2][2,1,1][0,0,1,1,1,1,0]$} & $\left(7, p^{2}-1\right) \neq 1$ & $\mathrm{SL}_{2}(p) \circ \mathrm{SL}_{2}(p)$ \\
\hline 9 & 5 & {$[3,2][1,2,2][1,0,1,1,1,1,0]$} & & $\mathrm{PSL}_{2}(\bar{p})$ \\
\hline 10 & 5 & {$[3,2][1,2,2][1,1,0,1,1,0,1]$} & & $\mathrm{PSL}_{2}(\bar{p})$ \\
\hline 11 & 5 & {$[3,2][1,2,2][1,1,1,0,0,1,1]$} & & $\mathrm{PSL}_{2}(\bar{p})$ \\
\hline 12 & 6 & {$[4,2][2,2,2][0,1,1,1,1,1,1]$} & & $\mathrm{SL}_{3}(2)$ \\
\hline 13 & 7 & {$[3,4][1,3,3][1,1,1,1,1,1,1]$} & & $\operatorname{PSL}(2,8)$ \\
\hline 14 & 8 & {$[4,4][2,3,3][1,1,1,0,2,1,2]$} & & $\mathrm{SL}_{2}(\bar{p}) \circ \mathrm{SL}_{2}(7)$ \\
\hline 15 & 8 & {$[4,4][2,3,3][1,2,1,1,1,2,0]$} & & $\mathrm{SL}_{2}(\bar{p}) \circ \mathrm{SL}_{2}(7)$ \\
\hline 16 & 8 & {$[4,4][2,3,3][1,1,2,2,1,0,1]$} & & $\mathrm{SL}_{2}(\bar{p}) \circ \mathrm{SL}_{2}(7)$ \\
\hline 17 & 8 & {$[4,4][2,3,3][0,2,1,1,1,1,2]$} & $\left(7, p^{2}-1\right)=1$ & $\operatorname{PSL}_{2}\left(p^{3}\right)$ \\
\hline $17^{\prime}$ & 8 & {$[4,4][2,3,3][0,2,1,1,1,1,2]$} & $\left(7, p^{2}-1\right) \neq 1$ & $\mathrm{SL}_{2}(p) \circ \mathrm{SL}_{2}(p)$ \\
\hline 18 & 8 & {$[4,4][2,3,3][0,1,2,1,1,2,1]$} & $\left(7, p^{2}-1\right)=1$ & $\mathrm{PSL}_{2}\left(p^{3}\right)$ \\
\hline $18^{\prime}$ & 8 & {$[4,4][2,3,3][0,1,2,1,1,2,1]$} & $\left(7, p^{2}-1\right) \neq 1$ & $\mathrm{SL}_{2}(p) \circ \mathrm{SL}_{2}(p)$ \\
\hline 19 & 8 & {$[4,4][2,3,3][0,1,1,2,2,1,1]$} & $\left(7, p^{2}-1\right)=1$ & $\operatorname{PSL}_{2}\left(p^{3}\right)$ \\
\hline $19^{\prime}$ & 8 & {$[4,4][2,3,3][0,1,1,2,2,1,1]$} & $\left(7, p^{2}-1\right) \neq 1$ & $\mathrm{SL}_{2}(p) \circ \mathrm{SL}_{2}(p)$ \\
\hline 20 & 8 & {$[4,4][2,3,3][1,2,1,2,0,1,1]$} & & $\mathrm{SL}_{2}(\bar{p}) \circ \mathrm{SL}_{2}(7)$ \\
\hline 21 & 8 & {$[4,4][2,3,3][1,0,2,1,1,1,2]$} & & $\mathrm{SL}_{2}(\bar{p}) \circ \mathrm{SL}_{2}(7)$ \\
\hline 22 & 8 & {$[4,4][2,3,3][1,1,0,1,2,2,1]$} & & $\mathrm{SL}_{2}(\bar{p}) \circ \mathrm{SL}_{2}(7)$ \\
\hline 23 & 9 & {$[5,4][3,3,3][1,2,2,2,1,1,0]$} & & $\mathrm{PSL}_{2}(\bar{p}) \times \mathrm{SL}_{3}(2)$ \\
\hline 24 & 9 & {$[5,4][3,3,3][1,1,2,1,2,0,2]$} & & $\mathrm{PSL}_{2}(\bar{p}) \times \mathrm{SL}_{3}(2)$ \\
\hline 25 & 9 & {$[5,4][3,3,3][1,1,2,2,0,1,2]$} & & $\mathrm{PSL}_{2}(\bar{p}) \times \mathrm{SL}_{3}(2)$ \\
\hline 26 & 9 & {$[5,4][3,3,3][1,2,0,2,1,2,1]$} & & $\mathrm{PSL}_{2}(\bar{p}) \times \mathrm{SL}_{3}(2)$ \\
\hline 27 & 9 & {$[5,4][3,3,3][1,2,1,0,2,2,1]$} & & $\mathrm{PSL}_{2}(\bar{p}) \times \mathrm{SL}_{3}(2)$ \\
\hline 28 & 9 & {$[5,4][3,3,3][1,0,1,1,2,2,2]$} & & $\mathrm{PSL}_{2}(\bar{p}) \times \mathrm{SL}_{3}(2)$ \\
\hline 29 & 13 & {$[7,6][4,3,6][1,2,2,2,2,2,2]$} & & $\mathrm{PSL}_{2}(27)$ \\
\hline 30 & 13 & {$[7,6][4,6,3][1,2,2,2,2,2,2]$} & & $\mathrm{PSL}_{2}(27)$ \\
\hline
\end{tabular}


Table A-2: $1<n \leqslant 13$ and $p=2$.

$\begin{array}{cccl}\text { no. } & \text { dim } & \text { multiplicity vector } & \text { image } \\ 1 & 2 & {[1,1][0,1,1][0,1,0,0,0,0,1]} & \mathrm{SL}_{2}(8) \\ 2 & 2 & {[1,1][0,1,1][0,0,1,0,0,1,0]} & \mathrm{SL}_{2}(8) \\ 3 & 2 & {[1,1][0,1,1][0,0,0,1,1,0,0]} & \mathrm{SL}_{2}(8) \\ 4 & 3 & {[2,1][1,1,1][0,1,1,0,1,0,0]} & \mathrm{SL}_{3}(2) \\ 5 & 3 & {[2,1][1,1,1][0,0,0,1,0,1,1]} & \mathrm{SL}_{3}(2) \\ 6 & 4 & {[2,2][2,1,1][0,1,1,0,0,1,1]} & \mathrm{SL}_{2}(8) \\ 7 & 4 & {[2,2][2,1,1][0,1,0,1,1,0,1]} & \mathrm{SL}_{2}(8) \\ 8 & 4 & {[2,2][2,1,1][0,0,1,1,1,1,0]} & \mathrm{SL}_{2}(8) \\ 9 & 6 & {[3,3][2,2,2][1,1,1,2,0,1,0]} & \mathrm{SL}_{2}(8) \times \mathrm{SL}_{3}(2) \\ 10 & 6 & {[3,3][2,2,2][1,0,1,0,2,1,1]} & \mathrm{SL}_{2}(8) \times \mathrm{SL}_{3}(2) \\ 11 & 6 & {[3,3][2,2,2][1,0,1,1,1,0,2]} & \mathrm{SL}_{2}(8) \times \mathrm{SL}_{3}(2) \\ 12 & 6 & {[3,3][2,2,2][1,2,0,1,1,1,0]} & \mathrm{SL}_{2}(8) \times \mathrm{SL}_{3}(2) \\ 13 & 6 & {[3,3][2,2,2][1,1,0,0,1,2,1]} & \mathrm{SL}_{2}(8) \times \mathrm{SL}_{3}(2) \\ 14 & 6 & {[3,3][2,2,2][1,1,2,1,0,0,1]} & \mathrm{SL}_{2}(8) \times \mathrm{SL}_{3}(2) \\ 15 & 13 & {[7,6][4,3,6][1,2,2,2,2,2,2]} & \mathrm{PSL}_{2}(27) \\ 16 & 13 & {[7,6][4,6,3][1,2,2,2,2,2,2]} & \mathrm{PSL}_{2}(27)\end{array}$

Table A-3: $1<n \leqslant 13$ and $p=3$.

\begin{tabular}{rrll} 
no. & $\operatorname{dim}$ & \multicolumn{1}{c}{ multiplicity vector } & image \\
1 & 3 & {$[1,2][1,1,1][1,1,0,0,0,0,1]$} & $\mathrm{PSL}_{2}(27)$ \\
2 & 3 & {$[1,2][1,1,1][1,0,1,0,0,1,0]$} & $\mathrm{PSL}_{2}(27)$ \\
3 & 3 & {$[1,2][1,1,1][1,0,0,1,1,0,0]$} & $\mathrm{PSL}_{2}(27)$ \\
4 & 3 & {$[1,2][1,1,1][0,1,1,0,1,0,0]$} & $\mathrm{SL}_{3}(2)$ \\
5 & 3 & {$[1,2][1,1,1][0,0,0,1,0,1,1]$} & $\mathrm{SL}_{3}(2)$ \\
6 & 4 & {$[2,2][2,1,1][0,1,1,0,0,1,1]$} & $\mathrm{PSL}_{2}(27)$ \\
7 & 4 & {$[2,2][2,1,1][0,1,0,1,1,0,1]$} & $\mathrm{PSL}_{2}(27)$ \\
8 & 4 & {$[2,2][2,1,1][0,0,1,1,1,1,0]$} & $\mathrm{PSL}_{2}(27)$ \\
9 & 6 & {$[4,2][2,2,2][0,1,1,1,1,1,1]$} & $\mathrm{SL}_{3}(2)$ \\
10 & 7 & {$[3,4][3,3,1][1,1,1,1,1,1,1]$} & $\mathrm{SL}_{2}(8)$ \\
11 & 8 & {$[4,4][3,3,2][1,2,1,2,0,1,1]$} & $\mathrm{SL}_{2}(27) \circ \mathrm{SL}_{2}(7)$ \\
12 & 8 & {$[4,4][3,3,2][1,0,2,1,1,1,2]$} & $\mathrm{SL}_{2}(27) \circ \mathrm{SL}_{2}(7)$ \\
13 & 8 & {$[4,4][3,3,2][1,1,0,1,2,2,1]$} & $\mathrm{SL}_{2}(27) \circ \mathrm{SL}_{2}(7)$ \\
14 & 8 & {$[4,4][3,3,2][1,1,1,0,2,1,2]$} & $\mathrm{SL}_{2}(27) \circ \mathrm{SL}_{2}(7)$ \\
15 & 8 & {$[4,4][3,3,2][1,2,1,1,1,2,0]$} & $\mathrm{SL}_{2}(27) \circ \mathrm{SL}_{2}(7)$ \\
16 & 8 & {$[4,4][3,3,2][1,1,2,2,1,0,1]$} & $\mathrm{SL}_{2}(27) \circ \mathrm{SL}_{2}(7)$ \\
17 & 9 & {$[5,4][3,3,3][1,2,2,2,1,1,0]$} & $\mathrm{PSL}_{2}(27) \times \mathrm{SL}_{3}(2)$ \\
18 & 9 & {$[5,4][3,3,3][1,1,2,1,2,0,2]$} & $\mathrm{PSL}_{2}(27) \times \mathrm{SL}_{3}(2)$ \\
19 & 9 & {$[5,4][3,3,3][1,1,2,2,0,1,2]$} & $\mathrm{PSL}_{2}(27) \times \mathrm{SL}_{3}(2)$ \\
20 & 9 & {$[5,4][3,3,3][1,2,0,2,1,2,1]$} & $\mathrm{PSL}_{2}(27) \times \mathrm{SL}_{3}(2)$ \\
21 & 9 & {$[5,4][3,3,3][1,2,1,0,2,2,1]$} & $\mathrm{PSL}_{2}(27) \times \mathrm{SL}_{3}(2)$ \\
22 & 9 & {$[5,4][3,3,3][1,0,1,1,2,2,2]$} & $\mathrm{PSL}_{2}(27) \times \mathrm{SL}_{3}(2)$ \\
& & &
\end{tabular}


Table A-4: $1<n \leqslant 13$ and $p=7$.

$\begin{array}{rrcl}\text { no. } & \operatorname{dim} & \text { multiplicity vector } & \text { image } \\ 1 & 3 & {[1,2][1,1,1][1,1,1,0,0,0,0]} & \operatorname{PSL}_{2}(7) \\ 2 & 5 & {[3,2][1,2,2][1,1,1,1,1,0,0]} & \operatorname{PSL}_{2}(7) \\ 3 & 7 & {[3,4][1,3,3][1,1,1,1,1,1,1]} & \mathrm{SL}_{2}(8) \\ 4 & 13 & {[7,6][4,3,6][2,2,2,2,2,2,1]} & \operatorname{PSL}(2,27) \\ 5 & 13 & {[7,6][4,6,3][2,2,2,2,2,2,1]} & \operatorname{PSL}(2,27)\end{array}$

Appendix B. Testing inequalities

Table B-1: $p \neq 2,3,7$.

ref. testing inequality

$T_{0} \quad a+m_{0} \leqslant b+c$

$T_{1} \quad m_{0}+m_{1}+m_{6} \leqslant a$

$T_{2} \quad m_{0}+m_{2}+m_{5} \leqslant a$

$T_{3} \quad m_{0}+m_{3}+m_{4} \leqslant a$

$T_{4} \quad m_{1}+m_{2}+m_{4} \leqslant a$

$T_{5} \quad m_{3}+m_{5}+m_{6} \leqslant a$

$T_{6} \quad m_{1}+m_{2}+m_{5}+m_{6} \leqslant b+c$

$T_{7} \quad m_{1}+m_{3}+m_{4}+m_{6} \leqslant b+c$

$T_{8} \quad m_{2}+m_{3}+m_{4}+m_{5} \leqslant b+c$

$T_{9} \quad a+b+c \leqslant n+m_{1}+m_{6}$

$T_{10} \quad a+b+c \leqslant n+m_{2}+m_{5}$

$T_{11} a+b+c \leqslant n+m_{3}+m_{4}$

$T_{12} \quad 2 a \leqslant n+m_{0}$

$T_{13} \quad 2 b+2 c \leqslant n+a$

$T_{14} \quad b+c+m_{1}+m_{6} \leqslant n+m_{0}$

$T_{15} \quad b+c+m_{2}+m_{5} \leqslant n+m_{0}$

$T_{16} \quad b+c+m_{3}+m_{4} \leqslant n+m_{0}$

$T_{17} \quad b+c+m_{1}+m_{3} \leqslant n+m_{4}$

$T_{18} \quad b+c+m_{2}+m_{6} \leqslant n+m_{1}$

$T_{19} \quad b+c+m_{4}+m_{5} \leqslant n+m_{2}$

$T_{20} \quad b+c+m_{4}+m_{6} \leqslant n+m_{3}$

$T_{21} \quad b+c+m_{1}+m_{5} \leqslant n+m_{6}$

$T_{22} \quad b+c+m_{2}+m_{3} \leqslant n+m_{5}$

$T_{23} \quad a+m_{1}+m_{2}+m_{3} \leqslant n+m_{6}$

$T_{24} \quad a+m_{2}+m_{4}+m_{6} \leqslant n+m_{5}$

$T_{25} \quad a+m_{2}+m_{3}+m_{6} \leqslant n+m_{4}$

$T_{26} \quad a+m_{1}+m_{3}+m_{5} \leqslant n+m_{2}$

$T_{27} \quad a+m_{1}+m_{4}+m_{5} \leqslant n+m_{3}$

$T_{28} \quad a+m_{4}+m_{5}+m_{6} \leqslant n+m_{1}$

$T_{29} \quad a+2 c \leqslant n+b+m_{0}$

$T_{30} \quad a+2 b \leqslant n+c+m_{0}$ warning reference

$n>1$ formula (5)

$n \neq 3 \quad$ Lemma 3.5

$n \neq 3 \quad$ Lemma 3.5

$n \neq 3 \quad$ Lemma 3.5

$n \neq 3 \quad$ Lemma 3.6

$n \neq 3 \quad$ Lemma 3.6

$n \neq 4 \quad$ Lemma 3.13

$n \neq 4 \quad$ Lemma 3.13

$n \neq 4 \quad$ Lemma 3.13

$n \neq 5 \quad$ Lemma 3.5

$n \neq 5 \quad$ Lemma 3.5

$n \neq 5 \quad$ Lemma 3.5

$n \neq 6 \quad$ Lemma 3.7

$n \neq 7 \quad$ Lemma 3.8

$n \neq 8 \quad$ Lemma 3.14

$n \neq 8 \quad$ Lemma 3.14

$n \neq 8 \quad$ Lemma 3.14

$n \neq 8 \quad$ Lemma 3.14

$n \neq 8 \quad$ Lemma 3.14

$n \neq 8 \quad$ Lemma 3.14

$n \neq 8 \quad$ Lemma 3.14

$n \neq 8 \quad$ Lemma 3.14

$n \neq 8 \quad$ Lemma 3.14

$n \neq 9 \quad$ Lemma 3.15

$n \neq 9 \quad$ Lemma 3.15

$n \neq 9 \quad$ Lemma 3.15

$n \neq 9 \quad$ Lemma 3.15

$n \neq 9 \quad$ Lemma 3.15

$n \neq 9 \quad$ Lemma 3.15

$n \neq 13 \quad$ Lemma 3.9

$n \neq 13 \quad$ Lemma 3.9 
Table B-2: $p=2$.

$\begin{array}{llll}\text { no. } & \text { testing inequality } & \text { warning } & \text { reference } \\ T_{0}^{2} & a+m_{0} \leqslant b+c & n>1 & \text { formula }(5) \\ T_{1}^{2} & b+c+m_{1}+m_{6} \leqslant n & n \neq 2 & \text { Lemma 3.5 } \\ T_{2} & b+c+m_{2}+m_{5} \leqslant n & n \neq 2 & \text { Lemma 3.5 } \\ T_{3}^{2} & b+c+m_{3}+m_{4} \leqslant n & n \neq 2 & \text { Lemma 3.5 } \\ T_{4}^{2} & a+m_{1}+m_{2}+m_{4} \leqslant n & n \neq 3 & \text { Lemma 3.6 } \\ T_{5}^{2} & a+m_{3}+m_{5}+m_{6} \leqslant n & n \neq 3 & \text { Lemma 3.6 } \\ T_{6}^{2} & m_{1}+m_{2}+m_{5}+m_{6} \leqslant b+c & n \neq 4 & \text { Lemma 3.13 } \\ T_{7}^{2} & m_{1}+m_{3}+m_{4}+m_{6} \leqslant b+c & n \neq 4 & \text { Lemma 3.13 } \\ T_{8}^{2} & m_{2}+m_{3}+m_{4}+m_{5} \leqslant b+c & n \neq 4 & \text { Lemma 3.13 } \\ T_{9}^{2} & m_{3} \leqslant m_{4}+m_{6} & n \neq 6 & \text { Lemma 3.14 } \\ T_{10}^{2} & m_{4} \leqslant m_{1}+m_{3} & n \neq 6 & \text { Lemma 3.14 } \\ T_{11}^{2} & m_{6} \leqslant m_{1}+m_{5} & n \neq 6 & \text { Lemma 3.14 } \\ T_{12}^{2} & m_{1} \leqslant m_{2}+m_{6} & n \neq 6 & \text { Lemma 3.14 } \\ T_{13}^{2} & m_{5} \leqslant m_{2}+m_{3} & n \neq 6 & \text { Lemma 3.14 } \\ T_{14}^{2} & m_{2} \leqslant m_{4}+m_{5} & n \neq 13 & \text { Lemma 3.9 } \\ T_{15} & a+2 c \leqslant n+b+m_{0} & n \neq 13 & \text { Lemma 3.9 } \\ T_{16}^{2} & a+2 b \leqslant n+c+m_{0} & & \end{array}$

Table B-3: $p=3$.

no. testing inequality

$T_{0}^{3} \quad a+m_{0} \leqslant b+c$

$T_{1}^{3} \quad m_{0}+m_{1}+m_{6} \leqslant a$

$T_{2}^{3} \quad m_{0}+m_{2}+m_{5} \leqslant a$

$T_{3}^{3} \quad m_{0}+m_{3}+m_{4} \leqslant a$

$T_{4}^{3} m_{1}+m_{2}+m_{4} \leqslant a$

$T_{5}^{3} \quad m_{3}+m_{5}+m_{6} \leqslant a$

$T_{6}^{3} \quad m_{1}+m_{2}+m_{5}+m_{6} \leqslant b+c$

$T_{7}^{3} \quad m_{1}+m_{3}+m_{4}+m_{6} \leqslant b+c$

$T_{8}^{3} \quad m_{2}+m_{3}+m_{4}+m_{5} \leqslant b+c$

$T_{9}^{3} \quad 2 a \leqslant n+m_{0}$

$T_{10}^{3} \quad n \leqslant a+2 c$

$T_{11}^{3} m_{1}+m_{3} \leqslant m_{4}+c$

$T_{12}^{3} \quad m_{2}+m_{6} \leqslant m_{1}+c$

$T_{13}^{3} m_{4}+m_{5} \leqslant m_{2}+c$

$T_{14}^{3} \quad m_{4}+m_{6} \leqslant m_{3}+c$

$T_{15}^{3} m_{1}+m_{5} \leqslant m_{6}+c$

$T_{16}^{3} \quad m_{2}+m_{3} \leqslant m_{5}+c$

$T_{17}^{3} \quad a+m_{1}+m_{2}+m_{3} \leqslant n+m_{6}$

$T_{18}^{3} \quad a+m_{2}+m_{4}+m_{6} \leqslant n+m_{5}$

$T_{19}^{3} \quad a+m_{2}+m_{3}+m_{6} \leqslant n+m_{4}$

$T_{20}^{3} \quad a+m_{1}+m_{3}+m_{5} \leqslant n+m_{2}$

$T_{21}^{3} \quad a+m_{1}+m_{4}+m_{5} \leqslant n+m_{3}$

$T_{22}^{3} \quad a+m_{4}+m_{5}+m_{6} \leqslant n+m_{1}$ warning

$n>1$

$n \neq 3$

$n \neq 3$

$n \neq 3$

$n \neq 3$

$n \neq 3$

$n \neq 4$

$n \neq 4$

$n \neq 4$

$n \neq 6$

$n \neq 7$

$n \neq 8$

$n \neq 8$

$n \neq 8$

$n \neq 8$

$n \neq 8$

$n \neq 8$

$n \neq 9$

$n \neq 9$

$n \neq 9$

$n \neq 9$

$n \neq 9$

$n \neq 9$ reference

formula (5)

Lemma 3.5

Lemma 3.5

Lemma 3.5

Lemma 3.6

Lemma 3.6

Lemma 3.13

Lemma 3.13

Lemma 3.13

Lemma 3.7

Lemma 3.8

Lemma 3.14

Lemma 3.14

Lemma 3.14

Lemma 3.14

Lemma 3.14

Lemma 3.14

Lemma 3.15

Lemma 3.15

Lemma 3.15

Lemma 3.15

Lemma 3.15

Lemma 3.15 
Table B-4: $p=7$.

$\begin{array}{llll}\text { no. } & \text { testing inequality } & \text { warning } & \\ T_{0}^{7} & a+m_{0} \leqslant b+c & n>1 & \text { formula }(5) \\ T_{1}^{7} & m_{0}+m_{1}+m_{2} \leqslant a & n \neq 3 & \text { Lemma 3.5 } \\ T_{2}^{7} & a+b+c \leqslant n+m_{5}+m_{6} & n \neq 5 & \text { Lemma 3.5 } \\ T_{3}^{7} & 2 b+2 c \leqslant n+a & n \neq 7 & \text { Lemma 3.8 } \\ T_{4}^{7} & a+2 c \leqslant n+b+m_{6} & n \neq 13 & \text { Lemma 3.9 } \\ T_{5}^{7} & a+2 b \leqslant n+c+m_{6} & n \neq 13 & \text { Lemma 3.9 }\end{array}$

NOTE: In all the tables below we omit multiplicity vectors that can be obtained from each other by substitutions $\omega \rightarrow \omega^{2}$ and $\varepsilon \rightarrow \varepsilon^{i}$ for $1 \leqslant i \leqslant 6$.

Appendix C. Admissible multiplicity vectors for $p \neq 2,3,7$

Table C-1: $1<n<20$.

\begin{tabular}{|c|c|c|c|}
\hline$n$ & multiplicity vector & rid. index & symm. type \\
\hline 3 & {$[1,2][1,1,1][1,1,0,0,0,0,1]$} & 0 & $(2,2)$ \\
\hline 3 & {$[1,2][1,1,1][0,1,1,0,1,0,0]$} & 0 & $(2,3)$ \\
\hline 8 & {$[4,4][2,3,3][1,2,1,2,0,1,1]$} & 0 & $(2,1)$ \\
\hline 9 & {$[5,4][3,3,3][1,2,2,2,1,1,0]$} & 0 & $(2,1)$ \\
\hline 12 & {$[6,6][4,4,4][1,1,2,1,3,2,2]$} & 2 & $(2,1)$ \\
\hline 12 & {$[6,6][4,4,4][2,1,2,3,1,1,2]$} & 2 & $(2,1)$ \\
\hline 13 & {$[7,6][4,3,6][1,2,2,2,2,2,2]$} & 0 & $(1,6)$ \\
\hline 14 & {$[6,8][5,3,6][2,2,2,2,2,2,2]$} & 0 & $(1,6)$ \\
\hline 15 & {$[7,8][4,4,7][3,2,2,2,2,2,2]$} & 0 & $(1,6)$ \\
\hline 15 & {$[7,8][5,5,5][2,1,3,2,2,2,3]$} & 4 & $(2,1)$ \\
\hline 16 & {$[8,8][6,5,5][2,1,2,2,3,3,3]$} & 4 & $(2,1)$ \\
\hline 16 & {$[8,8][5,4,7][2,2,2,3,3,2,2]$} & 2 & $(1,2)$ \\
\hline 16 & {$[8,8][4,6,6][2,1,2,2,3,3,3]$} & 2 & $(2,1)$ \\
\hline 16 & {$[8,8][6,5,5][2,1,3,1,3,2,4]$} & 0 & $(2,1)$ \\
\hline 16 & {$[8,8][5,4,7][2,1,2,2,3,3,3]$} & 0 & $(1,1)$ \\
\hline 16 & {$[8,8][5,4,7][3,1,2,3,2,3,2]$} & 0 & $(1,1)$ \\
\hline 16 & {$[8,8][5,4,7][3,1,3,2,2,2,3]$} & 0 & $(1,1)$ \\
\hline 17 & {$[9,8][5,6,6][2,2,2,3,2,3,3]$} & 6 & $(2,3)$ \\
\hline 17 & {$[9,8][5,6,6][2,1,3,3,3,2,3]$} & 4 & $(2,1)$ \\
\hline 17 & {$[9,8][5,6,6][3,1,2,2,3,3,3]$} & 4 & $(2,1)$ \\
\hline 17 & {$[9,8][5,6,6][2,2,2,2,3,4,2]$} & 4 & $(2,1)$ \\
\hline 17 & {$[9,8][6,4,7][2,2,2,3,2,3,3]$} & 2 & $(1,3)$ \\
\hline 17 & {$[9,8][5,6,6][1,2,3,4,2,2,3]$} & 2 & $(2,1)$ \\
\hline 17 & {$[9,8][5,6,6][3,1,2,3,2,2,4]$} & 2 & $(2,1)$ \\
\hline 17 & {$[9,8][6,4,7][2,1,3,3,3,2,3]$} & 0 & $(1,1)$ \\
\hline 17 & {$[9,8][6,4,7][2,2,2,2,3,4,2]$} & 0 & $(1,1)$ \\
\hline 18 & {$[10,8][6,6,6][2,2,3,4,2,2,3]$} & 4 & $(2,1)$ \\
\hline 18 & {$[10,8][6,6,6][2,1,3,3,2,3,4]$} & 2 & $(2,1)$ \\
\hline
\end{tabular}




$\begin{array}{llll}18 & {[10,8][6,6,6][2,2,2,5,2,3,2]} & 0 & (2,1) \\ 18 & {[8,10][5,5,8][2,2,3,3,3,3,2]} & 0 & (1,2) \\ 18 & {[10,8][5,5,8][3,2,2,3,2,3,3]} & 0 & (1,3) \\ 18 & {[10,8][6,6,6][2,1,2,4,3,2,4]} & 0 & (2,1) \\ 18 & {[8,10][6,6,6][2,1,3,2,4,2,4]} & 0 & (2,1) \\ 19 & {[9,10][7,6,6][2,2,3,2,4,3,3]} & 6 & (2,1) \\ 19 & {[9,10][7,6,6][3,2,2,2,3,3,4]} & 6 & (2,1) \\ 19 & {[9,10][6,5,8][3,2,3,3,3,3,2]} & 4 & (1,2) \\ 19 & {[9,10][5,7,7][2,2,3,2,4,3,3]} & 4 & (2,1) \\ 19 & {[9,10][5,7,7][3,2,2,2,3,3,4]} & 4 & (2,1) \\ 19 & {[9,10][6,5,8][1,3,3,3,3,3,3]} & 2 & (1,6) \\ 19 & {[9,10][6,5,8][4,2,2,3,2,3,3]} & 2 & (1,3) \\ 19 & {[9,10][7,6,6][3,1,3,2,4,2,4]} & 2 & (2,1) \\ 19 & {[9,10][6,5,8][2,2,3,2,4,3,3]} & 2 & (1,1) \\ 19 & {[9,10][6,5,8][3,2,2,2,3,3,4]} & 2 & (1,1) \\ 19 & {[9,10][6,5,8][2,1,4,3,3,3,3]} & 0 & (1,1) \\ 19 & {[9,10][6,5,8][2,2,2,3,4,4,2]} & 0 & (1,1)\end{array}$

Table C-2: Admissible multiplicity vectors with symmetries $(2,6),(1,6),(2,3),(2,2),(1,3)$, and $(1,2)$ for $19<n<32$ (for $n=31$ the vectors with symmetries $(1,2)$ and $(1,3)$ are not included).

$\begin{array}{rrcc}n & \text { multiplicity vector } & \text { rid. index } & \text { symm. type } \\ 20 & {[10,10][6,7,7][2,3,3,3,3,3,3]} & 10 & (2,6) \\ 20 & {[10,10][5,6,9][2,3,3,3,3,3,3]} & 2 & (1,6) \\ 20 & {[10,10][7,5,8][2,3,3,3,3,3,3]} & 6 & (1,6) \\ 21 & {[11,10][7,7,7][3,3,3,3,3,3,3]} & 12 & (2,6) \\ 21 & {[11,10][6,6,9][3,3,3,3,3,3,3]} & 6 & (1,6) \\ 21 & {[9,12][6,6,9][3,3,3,3,3,3,3]} & 2 & (1,6) \\ 22 & {[10,12][7,6,9][4,3,3,3,3,3,3]} & 6 & (1,6) \\ 22 & {[10,12][7,6,9][2,3,3,4,4,3,3]} & 4 & (1,2) \\ 22 & {[12,10][7,6,9][2,3,3,4,4,3,3]} & 4 & (1,2) \\ 22 & {[12,10][6,8,8][4,2,2,4,2,4,4]} & 2 & (2,3) \\ 23 & {[11,12][7,8,8][3,3,3,4,4,3,3]} & 12 & (2,2) \\ 23 & {[11,12][6,7,10][3,3,3,4,4,3,3]} & 4 & (1,2) \\ 23 & {[11,12][8,6,9][3,3,3,4,4,3,3]} & 8 & (1,2) \\ 24 & {[12,12][6,9,9][3,3,3,4,3,4,4]} & 8 & (2,3) \\ 24 & {[12,12][8,8,8][3,3,3,4,3,4,4]} & 14 & (2,3) \\ 24 & {[12,12][7,7,10][3,3,3,4,3,4,4]} & 8 & (1,3) \\ 24 & {[12,12][9,6,9][3,3,3,4,3,4,4]} & 8 & (1,3) \\ 24 & {[12,12][7,7,10][3,3,3,4,3,4,4]} & 8 & (1,3) \\ 24 & {[12,12][8,8,8][4,3,3,4,4,3,3]} & 14 & (2,2) \\ 24 & {[12,12][8,8,8][2,3,4,4,4,4,3]} & 12 & (2,2) \\ 24 & {[12,12][7,7,10][4,3,3,4,4,3,3]} & 8 & (1,2) \\ 24 & {[12,12][7,7,10][2,3,4,4,4,4,3]} & 6 & (1,2) \\ 25 & {[13,12][8,7,10][1,4,4,4,4,4,4]} & 4 & (1,6)\end{array}$


$[14,14][8,10,10][4,4,4,4,4,4,4]$ 
30

30

30

30

30

30

30

30

30

30

30

30

30

30

30

30

30

30

30
$[14,14][8,10,10][2,4,4,5,5,4,4]$ $[14,14][10,9,9][4,3,4,5,5,4,3]$ $[14,14][10,9,9][4,3,5,4,4,5,3]$ $[14,14][9,8,11][4,3,4,5,5,4,3]$ $[14,14][9,8,11][4,3,5,4,4,5,3]$ $[14,14][9,8,11][2,4,4,5,5,4,4]$ $[13,16][9,10,10][5,4,4,4,4,4,4]$ $[15,14][9,10,10][5,4,4,4,4,4,4]$ $[13,16][8,9,12][5,4,4,4,4,4,4]$ $[13,16][10,8,11][5,4,4,4,4,4,4]$ $[15,14][8,9,12][5,4,4,4,4,4,4]$ $[15,14][9,7,13][5,4,4,4,4,4,4]$ $[15,14][9,10,10][2,4,4,5,4,5,5]$ $[15,14][9,10,10][5,3,3,5,3,5,5]$ $[15,14][11,9,9][2,4,4,5,4,5,5]$ $[15,14][7,11,11][5,3,3,5,3,5,5]$ $[15,14][9,10,10][3,4,4,5,5,4,4]$ $[15,14][11,9,9][3,4,4,5,5,4,4]$ $[15,14][9,10,10][5,3,4,5,5,4,3]$ $[15,14][9,10,10][5,3,5,4,4,5,3]$ $[15,14][9,10,10][3,3,5,5,5,5,3]$ $[13,16][9,10,10][3,4,4,5,5,4,4]$ $[15,14][8,9,12][2,4,4,5,4,5,5]$ $[15,14][10,8,11][2,4,4,5,4,5,5]$ $[15,14][8,9,12][5,3,3,5,3,5,5]$ $[15,14][10,8,11][3,4,4,5,5,4,4]$ $[13,16][10,8,11][3,4,4,5,5,4,4]$ $[15,14][8,9,12][3,4,4,5,5,4,4]$ $[13,16][8,9,12][3,4,4,5,5,4,4]$ $[14,16][10,10,10][6,4,4,4,4,4,4]$ $[16,14][10,10,10][3,4,4,5,4,5,5]$ $[16,14][8,11,11][3,4,4,5,4,5,5]$ $[16,14][8,11,11][6,3,3,5,3,5,5]$ $[14,16][10,10,10][4,4,4,5,5,4,4]$ $[16,14][10,10,10][4,4,4,5,5,4,4]$ $[14,16][10,10,10][4,3,5,5,5,5,3]$ $[16,14][10,10,10][4,3,5,5,5,5,3]$ $[16,14][8,11,11][4,4,4,5,5,4,4]$ $[14,16][8,11,11][4,4,4,5,5,4,4]$ $[14,16][10,10,10][2,4,5,5,5,5,4]$ $[16,14][10,10,10][2,4,5,5,5,5,4]$ $[16,14][7,10,13][3,4,4,5,4,5,5]$ $[16,14][9,9,12][3,4,4,5,4,5,5]$ $[16,14][11,8,11][3,4,4,5,4,5,5]$ $[14,16][9,9,12][4,4,4,5,5,4,4]$ $[14,16][11,8,11][4,4,4,5,5,4,4]$ $[16,14][9,9,12][4,4,4,5,5,4,4]$ $[14,16][9,9,12][4,3,5,5,5,5,3]$
14

16

16

12

12

10

16

20

8

12

12

8

14

14

12

4

18

16

16

16

14

14

6

10

6

14

10

10

6

18

18

12

6

20

20

16

16

12

12

14

14

0

12

12

14

14

14

10 


$\begin{array}{lrcc}30 & {[14,16][9,9,12][2,4,5,5,5,5,4]} & 8 & (1,2) \\ 30 & {[16,14][9,9,12][2,4,5,5,5,5,4]} & 8 & (1,2) \\ 31 & {[15,16][11,10,10][4,4,4,5,4,5,5]} & 22 & (2,3) \\ 31 & {[15,16][9,11,11][4,4,4,5,4,5,5]} & 20 & (2,3) \\ 31 & {[17,14][9,11,11][4,4,4,5,4,5,5]} & 16 & (2,3) \\ 31 & {[15,16][9,11,11][7,3,3,5,3,5,5]} & 8 & (2,3) \\ 31 & {[15,16][11,10,10][5,4,4,5,5,4,4]} & 22 & (2,2) \\ 31 & {[15,16][9,11,11][5,4,4,5,5,4,4]} & 20 & (2,2) \\ 31 & {[15,16][11,10,10][3,4,5,5,5,5,4]} & 20 & (2,2) \\ 31 & {[15,16][9,11,11][3,4,5,5,5,5,4]} & 18 & (2,2) \\ 31 & {[15,16][11,10,10][5,3,5,5,5,5,3]} & 18 & (2,2) \\ 31 & {[17,14][9,11,11][5,4,4,5,5,4,4]} & 16 & (2,2) \\ 31 & {[17,14][11,10,10][3,4,5,5,5,5,4]} & 16 & (2,2) \\ 31 & {[15,16][9,11,11][5,3,5,5,5,5,3]} & 16 & (2,2) \\ 31 & {[15,16][11,10,10][3,4,4,6,6,4,4]} & 16 & (2,2) \\ 31 & {[17,14][9,11,11][3,4,5,5,5,5,4]} & 14 & (2,2) \\ 31 & {[15,16][9,11,11][3,4,4,6,6,4,4]} & 14 & (2,2)\end{array}$

Table C-3: Admissible multiplicity vectors with $(2,6)$ symmetry for $31<n<41$.

$\begin{array}{ccc}n & \text { multiplicity vector } & \text { rid. index } \\ 32 & {[16,16][12,10,10][2,5,5,5,5,5,5]} & 16 \\ 32 & {[16,16][10,11,11][2,5,5,5,5,5,5]} & 18 \\ 33 & {[15,18][9,12,12][3,5,5,5,5,5,5]} & 14 \\ 33 & {[17,16][9,12,12][3,5,5,5,5,5,5]} & 18 \\ 33 & {[17,16][13,10,10][3,5,5,5,5,5,5]} & 18 \\ 33 & {[15,18][11,11,11][3,5,5,5,5,5,5]} & 20 \\ 33 & {[17,16][11,11,11][3,5,5,5,5,5,5]} & 24 \\ 34 & {[16,18][10,12,12][4,5,5,5,5,5,5]} & 24 \\ 34 & {[18,16][10,12,12][4,5,5,5,5,5,5]} & 24 \\ 34 & {[16,18][12,11,11][4,5,5,5,5,5,5]} & 26 \\ 34 & {[18,16][12,11,11][4,5,5,5,5,5,5]} & 26 \\ 35 & {[19,16][11,12,12][5,5,5,5,5,5,5]} & 26 \\ 35 & {[19,16][9,13,13][5,5,5,5,5,5,5]} & 16 \\ 35 & {[15,20][11,12,12][5,5,5,5,5,5,5]} & 18 \\ 35 & {[17,18][9,13,13][5,5,5,5,5,5,5]} & 20 \\ 35 & {[17,18][13,11,11][5,5,5,5,5,5,5]} & 28 \\ 35 & {[17,18][11,12,12][5,5,5,5,5,5,5]} & 30 \\ 36 & {[16,20][10,13,13][6,5,5,5,5,5,5]} & 18 \\ 36 & {[20,16][10,13,13][6,5,5,5,5,5,5]} & 18 \\ 36 & {[16,20][12,12,12][6,5,5,5,5,5,5]} & 24 \\ 36 & {[18,18][10,13,13][6,5,5,5,5,5,5]} & 26 \\ 36 & {[18,18][12,12,12][6,5,5,5,5,5,5]} & 32 \\ 37 & {[17,20][11,13,13][7,5,5,5,5,5,5]} & 24 \\ 37 & {[17,20][13,12,12][7,5,5,5,5,5,5]} & 26 \\ & & \end{array}$




$\begin{array}{lll}37 & {[19,18][11,13,13][7,5,5,5,5,5,5]} & 28 \\ 38 & {[18,20][14,12,12][2,6,6,6,6,6,6]} & 18 \\ 38 & {[20,18][14,12,12][2,6,6,6,6,6,6]} & 18 \\ 38 & {[18,20][12,13,13][2,6,6,6,6,6,6]} & 20 \\ 38 & {[20,18][12,13,13][2,6,6,6,6,6,6]} & 20 \\ 38 & {[18,20][12,13,13][8,5,5,5,5,5,5]} & 26 \\ 39 & {[19,20][11,14,14][3,6,6,6,6,6,6]} & 24 \\ 39 & {[19,20][13,13,13][3,6,6,6,6,6,6]} & 30 \\ 39 & {[19,20][15,12,12][3,6,6,6,6,6,6]} & 24 \\ 39 & {[21,18][11,14,14][3,6,6,6,6,6,6]} & 20 \\ 39 & {[21,18][13,13,13][3,6,6,6,6,6,6]} & 26 \\ 39 & {[21,18][15,12,12][3,6,6,6,6,6,6]} & 20 \\ 40 & {[18,22][12,14,14][4,6,6,6,6,6,6]} & 26 \\ 40 & {[18,22][14,13,13][4,6,6,6,6,6,6]} & 28 \\ 40 & {[20,20][10,15,15][4,6,6,6,6,6,6]} & 20 \\ 40 & {[20,20][12,14,14][4,6,6,6,6,6,6]} & 34 \\ 40 & {[20,20][14,13,13][4,6,6,6,6,6,6]} & 36 \\ 40 & {[20,20][16,12,12][4,6,6,6,6,6,6]} & 26 \\ 40 & {[22,18][12,14,14][4,6,6,6,6,6,6]} & 26 \\ 40 & {[22,18][14,13,13][4,6,6,6,6,6,6]} & 28\end{array}$

Appendix D. Admissible multiplicity vectors for $p=2$

Table D-1: $1<n<20$.

$\begin{array}{rccc}n & \text { multiplicity vector } & \text { rid. index } & \text { symm. type } \\ 3 & {[2,1][1,1,1][0,1,1,0,1,0,0]} & 0 & (2,1) \\ 6 & {[3,3][2,2,2][1,1,1,2,0,1,0]} & 0 & (2,1) \\ 12 & {[6,6][4,4,4][2,1,1,1,2,2,3]} & 2 & (2,1) \\ 13 & {[7,6][4,3,6][1,2,2,2,2,2,2]} & 0 & (1,6) \\ 14 & {[7,7][5,3,6][2,2,2,2,2,2,2]} & 2 & (1,6) \\ 15 & {[8,7][4,4,7][3,2,2,2,2,2,2]} & 0 & (1,6) \\ 15 & {[8,7][5,5,5][2,1,3,2,2,2,3]} & 4 & (2,1) \\ 16 & {[8,8][5,4,7][2,1,2,2,3,3,3]} & 0 & (1,1) \\ 16 & {[8,8][5,4,7][3,1,2,3,2,3,2]} & 0 & (1,1) \\ 16 & {[8,8][5,4,7][3,1,3,2,2,2,3]} & 0 & (1,1) \\ 16 & {[8,8][6,5,5][2,1,2,2,3,3,3]} & 4 & (2,1) \\ 17 & {[9,8][6,4,7][2,1,3,3,3,2,3]} & 0 & (1,1) \\ 17 & {[9,8][6,4,7][2,2,2,2,3,4,2]} & 0 & (1,1) \\ 17 & {[9,8][5,6,6][3,1,2,2,3,3,3]} & 4 & (2,1) \\ 18 & {[9,9][7,4,7][2,2,2,2,3,3,4]} & 0 & (1,1) \\ 18 & {[9,9][5,5,8][3,2,2,3,2,3,3]} & 2 & (1,3) \\ 18 & {[9,9][6,6,6][1,2,3,2,4,3,3]} & 4 & (2,1) \\ 18 & {[9,9][6,6,6][3,1,3,3,3,2,3]} & 6 & (2,1) \\ 18 & {[9,9][6,6,6][2,2,2,2,3,3,4]} & 6 & (2,1) \\ 18 & {[9,9][6,6,6][3,2,2,2,3,4,2]} & 6 & (2,1) \\ 18 & {[9,9][6,6,6][3,2,2,3,2,3,3]} & 8 & (2,3)\end{array}$


$19 \quad[10,9][7,6,6][2,2,3,2,4,3,3] \quad 6$

$19 \quad[10,9][6,5,8][3,2,3,3,3,3,2] \quad 4$

$19 \quad[10,9][6,5,8][2,2,3,2,4,3,3] \quad 2$

$19 \quad[10,9][6,5,8][3,2,2,2,3,3,4] \quad 2$

Table D-2: Admissible multiplicity vectors with non-trivial symmetry for $19<n<33$.

multiplicity vector $[10,10][7,5,8][2,3,3,3,3,3,3]$ $[11,9][7,5,8][2,3,3,3,3,3,3]$ $[11,10][7,7,7][3,3,3,3,3,3,3]$ $[11,10][6,6,9][3,3,3,3,3,3,3]$ $[12,9][6,6,9][3,3,3,3,3,3,3]$ $[11,11][7,6,9][4,3,3,3,3,3,3]$ $[11,10][8,6,9][3,3,3,4,4,3,3]$ $[12,12][8,8,8][3,3,3,4,3,4,4]$ $[12,12][8,8,8][4,3,3,4,4,3,3]$ $[12,12][8,8,8][2,3,4,4,4,4,3]$ $[12,12][7,7,10][3,3,3,4,3,4,4]$ $[12,12][9,6,9][3,3,3,4,3,4,4]$ $[12,12][9,6,9][3,3,3,4,4,3,3]$ $[13,12][7,9,9][4,3,3,4,3,4,4]$ $[13,12][9,8,8][3,3,4,4,4,4,3]$ $[13,12][8,7,10][4,3,3,4,3,4,4]$ $[14,11][8,7,10][3,3,4,4,4,4,3]$ $[13,12][8,7,10][3,3,4,4,4,4,3]$ $[14,12][9,7,10][2,4,4,4,4,4,4]$ $[13,13][9,7,10][2,4,4,4,4,4,4]$ $[13,13][8,9,9][5,3,3,4,3,4,4]$ $[14,12][8,9,9][4,3,4,4,4,4,3]$ $[13,13][8,9,9][4,3,4,4,4,4,3]$ $[14,12][7,8,11][5,3,3,4,3,4,4]$ $[13,13][7,8,11][5,3,3,4,3,4,4]$ $[13,13][9,7,10][4,3,4,4,4,4,3]$ $[15,12][9,9,9][3,4,4,4,4,4,4]$ $[14,13][9,9,9][3,4,4,4,4,4,4]$ $[15,12][8,8,11][3,4,4,4,4,4,4]$ $[14,13][8,8,11][3,4,4,4,4,4,4]$ $[15,12][10,7,10][3,4,4,4,4,4,4]$ $[14,14][10,9,9][4,4,4,4,4,4,4]$ $[14,14][8,10,10][4,4,4,4,4,4,4]$ $[15,13][8,10,10][4,4,4,4,4,4,4]$ $[15,13][9,8,11][4,4,4,4,4,4,4]$ $[14,14][9,8,11][4,4,4,4,4,4,4]$ $[14,14][10,6,12][4,4,4,4,4,4,4]$ rid. index symm. type 
$31 \quad[17,14][11,10,10][3,4,5,5,5,5,4]$

32

$[17,15][11,9,12][2,5,5,5,5,5,5]$

32

$[17,15][10,11,11][5,4,4,5,4,5,5]$

32

$[16,16][12,10,10][2,4,4,6,4,6,6]$

$32 \quad[17,15][12,10,10][2,4,4,6,4,6,6]$

32

$[16,16][10,11,11][4,4,5,5,5,5,4]$

$32 \quad[16,16][12,10,10][4,4,5,5,5,5,4]$

32

32

$[16,16][10,11,11][6,4,4,5,5,4,4]$

32

$[17,15][10,11,11][4,4,5,5,5,5,4]$

$32 \quad[16,16][12,10,10][4,4,4,6,6,4,4]$

32

$[18,14][10,11,11][4,4,5,5,5,5,4]$

$(2,2)$

Table D-3: Admissible multiplicity vectors with $(2,6)$ symmetry for $32<n<41$.

$\begin{array}{lcc}n & \text { multiplicity vector } & \text { rid. index } \\ 33 & {[17,16][13,10,10][3,5,5,5,5,5,5]} & 18 \\ 33 & {[18,15][11,11,11][3,5,5,5,5,5,5]} & 20 \\ 33 & {[17,16][11,11,11][3,5,5,5,5,5,5]} & 24 \\ 34 & {[17,17][12,11,11][4,5,5,5,5,5,5]} & 28 \\ 34 & {[17,17][10,12,12][4,5,5,5,5,5,5]} & 26 \\ 34 & {[18,16][12,11,11][4,5,5,5,5,5,5]} & 26 \\ 34 & {[18,16][10,12,12][4,5,5,5,5,5,5]} & 24 \\ 34 & {[18,16][10,12,12][4,5,5,5,5,5,5]} & 18\end{array}$




$\begin{array}{lll}35 & {[18,17][11,12,12][5,5,5,5,5,5,5]} & 30 \\ 35 & {[19,16][11,12,12][5,5,5,5,5,5,5]} & 26 \\ 36 & {[20,16][10,13,13][6,5,5,5,5,5,5]} & 18 \\ 36 & {[18,18][10,13,13][6,5,5,5,5,5,5]} & 26 \\ 36 & {[18,18][12,12,12][6,5,5,5,5,5,5]} & 32 \\ 37 & {[19,18][11,13,13][7,5,5,5,5,5,5]} & 28 \\ 39 & {[20,19][13,13,13][3,6,6,6,6,6,6]} & 30 \\ 39 & {[20,19][15,12,12][3,6,6,6,6,6,6]} & 24 \\ 39 & {[21,18][13,13,13][3,6,6,6,6,6,6]} & 26 \\ 39 & {[21,18][15,12,12][3,6,6,6,6,6,6]} & 20 \\ 40 & {[22,18][12,14,14][4,6,6,6,6,6,6]} & 26 \\ 40 & {[22,18][14,13,13][4,6,6,6,6,6,6]} & 28 \\ 40 & {[21,19][12,14,14][4,6,6,6,6,6,6]} & 32 \\ 40 & {[21,19][14,13,13][4,6,6,6,6,6,6]} & 34 \\ 40 & {[20,20][12,14,14][4,6,6,6,6,6,6]} & 34 \\ 40 & {[20,20][14,13,13][4,6,6,6,6,6,6]} & 36 \\ 40 & {[20,20][16,12,12][4,6,6,6,6,6,6]} & 26\end{array}$

Table D-4: Admissible symplectic multiplicity vectors for $p=2$ and $2<n<30$.

multiplicity vector $[3,3][2,2,2][0,1,1,1,1,1,1]$ $[7,7][4,5,5][2,2,2,2,2,2,2]$ $[9,9][6,6,6][2,2,3,3,3,3,2]$ $[10,10][6,7,7][2,3,3,3,3,3,3]$ $[11,9][6,7,7][2,3,3,3,3,3,3]$ $[11,11][6,8,8][4,3,3,3,3,3,3]$ $[11,11][8,7,7][2,3,3,4,4,3,3]$ $[12,12][8,8,8][4,3,3,4,4,3,3]$ $[12,12][8,8,8][2,3,4,4,4,4,3]$ $[13,11][8,8,8][2,3,4,4,4,4,3]$ $[13,13][8,9,9][2,4,4,4,4,4,4]$ $[13,13][10,8,8][2,4,4,4,4,4,4]$ $[14,12][8,9,9][2,4,4,4,4,4,4]$ $[13,13][8,9,9][4,3,4,4,4,4,3]$ $[14,12][8,9,9][4,3,4,4,4,4,3]$ $[14,14][10,9,9][4,4,4,4,4,4,4]$ $[14,14][8,10,10][4,4,4,4,4,4,4]$ $[15,13][8,10,10][4,4,4,4,4,4,4]$ $[14,14][10,9,9][4,3,4,5,5,4,3]$ $[14,14][10,9,9][4,3,5,4,4,5,3]$ $[14,14][10,9,9][2,4,4,5,5,4,4]$ $[15,13][10,9,9][2,4,4,5,5,4,4]$ rid. ind

2

6

8

10

8

10

10

14

12

10

14

12

12

16

14

20

18

16

16

16

14

12
30

26

18

32

28

30

26

20

26

32

34

34

26 
Appendix E. Admissible multiplicity vectors for $p=3$

Table E-1: $1<n<20$.

$\begin{array}{lccc}n & \text { multiplicity vector } & \text { rid. index } & \text { symm. type } \\ 3 & {[1,2][1,1,1][0,1,1,0,1,0,0]} & 0 & (*, 3) \\ 8 & {[4,4][3,3,2][1,2,1,2,0,1,1]} & 0 & (*, 1) \\ 9 & {[5,4][3,3,3][1,2,2,2,1,1,0]} & 0 & (*, 1) \\ 12 & {[6,6][4,4,4][1,1,2,1,3,2,2]} & 2 & (*, 1) \\ 12 & {[6,6][4,4,4][2,1,1,1,2,2,3]} & 2 & (*, 1) \\ 15 & {[7,8][5,5,5][2,1,3,2,2,2,3]} & 4 & (*, 1) \\ 16 & {[8,8][6,6,4][2,1,2,2,3,3,3]} & 2 & (*, 1) \\ 16 & {[8,8][6,5,5][2,1,2,2,3,3,3]} & 4 & (*, 1) \\ 17 & {[9,8][6,6,5][2,2,2,3,2,3,3]} & 6 & (*, 3) \\ 17 & {[9,8][6,6,5][2,1,3,3,3,2,3]} & 4 & (*, 1) \\ 17 & {[9,8][6,6,5][2,2,2,2,3,4,2]} & 4 & (*, 1) \\ 17 & {[9,8][6,6,5][1,2,2,2,3,3,4]} & 2 & (*, 1) \\ 18 & {[10,8][6,6,6][2,2,2,2,3,3,4]} & 4 & (*, 1) \\ 18 & {[10,8][6,6,6][2,1,3,3,2,3,4]} & 2 & (*, 1) \\ 18 & {[10,8][6,6,6][2,1,2,4,3,2,4]} & 0 & (*, 1) \\ 18 & {[10,8][6,6,6][2,1,3,2,4,2,4]} & 0 & (*, 1) \\ 18 & {[8,10][6,6,6][2,1,3,2,4,2,4]} & 0 & (*, 1) \\ 19 & {[9,10][7,6,6][2,2,3,2,4,3,3]} & 6 & (*, 1) \\ 19 & {[9,10][7,6,6][3,2,2,2,3,3,4]} & 6 & (*, 1) \\ 19 & {[9,10][7,7,5][2,2,3,2,4,3,3]} & 4 & (*, 1) \\ 19 & {[9,10][7,7,5][3,2,2,2,3,3,4]} & 4 & (*, 1) \\ 19 & {[9,10][7,7,5][3,1,3,2,4,2,4]} & 2 & (*, 1)\end{array}$

Table E-2: Admissible multiplicity vectors

with symmetries $(*, 6),(*, 3)$ and $(*, 2)$ for $19<n<30$.

$\begin{array}{lccc}n & \text { multiplicity vector } & \text { rid. index } & \text { symm. type } \\ 20 & {[10,10][7,7,6][2,3,3,3,3,3,3]} & 10 & (*, 6) \\ 21 & {[11,10][7,7,7][3,3,3,3,3,3,3]} & 12 & (*, 6) \\ 23 & {[11,12][8,8,7][3,3,3,4,4,3,3]} & 12 & (*, 2) \\ 24 & {[12,12][8,8,8][3,3,3,4,3,4,4]} & 14 & (*, 3) \\ 24 & {[12,12][9,8,7][3,3,3,4,3,4,4]} & 12 & (*, 3) \\ 24 & {[12,12][10,7,7][3,3,3,4,3,4,4]} & 8 & (*, 3) \\ 24 & {[12,12][8,8,8][4,3,3,4,4,3,3]} & 14 & (*, 2) \\ 24 & {[12,12][8,8,8][2,3,4,4,4,4,3]} & 12 & (*, 2) \\ 25 & {[13,12][9,9,7][3,3,4,4,4,4,3]} & 12 & (*, 2) \\ 25 & {[13,12][9,8,8][3,3,4,4,4,4,3]} & 14 & (*, 2) \\ 26 & {[14,12][10,8,8][2,4,4,4,4,4,4]} & 12 & (*, 6) \\ 26 & {[12,14][10,8,8][2,4,4,4,4,4,4]} & 12 & (*, 6) \\ 26 & {[12,14][10,8,8][5,3,3,4,3,4,4]} & 12 & (*, 3) \\ 26 & {[12,14][10,8,8][4,3,4,4,4,4,3]} & 14 & (*, 2)\end{array}$




$\begin{array}{lrcr}27 & {[13,14][10,10,7][3,4,4,4,4,4,4]} & 12 & (*, 6) \\ 27 & {[13,14][10,9,8][3,4,4,4,4,4,4]} & 16 & (*, 6) \\ 27 & {[13,14][9,9,9][3,4,4,4,4,4,4]} & 18 & (*, 6) \\ 27 & {[15,12][9,9,9][3,4,4,4,4,4,4]} & 14 & (*, 6) \\ 27 & {[15,12][9,9,9][3,3,3,4,3,4,4]} & 8 & (*, 3) \\ 27 & {[13,14][9,9,9][5,3,4,4,4,4,3]} & 16 & (*, 2) \\ 28 & {[14,14][10,10,8][4,4,4,4,4,4,4]} & 18 & (*, 6) \\ 28 & {[14,14][10,9,9][4,4,4,4,4,4,4]} & 20 & (*, 6) \\ 28 & {[14,14][10,10,8][4,3,4,5,5,4,3]} & 14 & (*, 2) \\ 28 & {[14,14][10,9,9][4,3,4,5,5,4,3]} & 16 & (*, 2) \\ 28 & {[14,14][10,10,8][4,3,5,4,4,5,3]} & 14 & (*, 2) \\ 28 & {[14,14][10,9,9][4,3,5,4,4,5,3]} & 16 & (*, 2) \\ 28 & {[14,14][10,10,8][2,4,4,5,5,4,4]} & 12 & (*, 2) \\ 28 & {[14,14][10,9,9][2,4,4,5,5,4,4]} & 14 & (*, 2) \\ 29 & {[13,16][10,10,9][5,4,4,4,4,4,4]} & 16 & (*, 6) \\ 29 & {[15,14][11,10,8][2,4,4,5,4,5,5]} & 10 & (*, 3) \\ 29 & {[15,14][11,9,9][2,4,4,5,4,5,5]} & 12 & (*, 3) \\ 29 & {[15,14][10,10,9][2,4,4,5,4,5,5]} & 14 & (*, 3) \\ 29 & {[13,16][10,10,9][3,4,4,5,5,4,4]} & 14 & (*, 2) \\ 29 & {[15,14][11,10,8][3,4,4,5,5,4,4]} & 14 & (*, 2) \\ 29 & {[15,14][11,9,9][3,4,4,5,5,4,4]} & 16 & (*, 2) \\ 29 & {[15,14][10,10,9][3,4,4,5,5,4,4]} & 18 & (*, 2)\end{array}$

Table E-3: Admissible multiplicity vectors with $(*, 3)$ and $(*, 6)$ symmetry for $29<n<34$.

$\begin{array}{lccc}n & \text { multiplicity vector } & \text { rid. index } & \text { symm. type } \\ 30 & {[14,16][10,10,10][6,4,4,4,4,4,4]} & 18 & \left(^{*}, 6\right) \\ 30 & {[16,14][10,10,10][3,4,4,5,4,5,5]} & 18 & \left(^{*}, 3\right) \\ 30 & {[16,14][11,10,9][3,4,4,5,4,5,5]} & 16 & \left(^{*}, 3\right) \\ 30 & {[16,14][11,11,8][3,4,4,5,4,5,5]} & 12 & \left(^{*}, 3\right) \\ 31 & {[15,16][11,11,9][4,4,4,5,4,5,5]} & 20 & \left(^{*}, 3\right) \\ 31 & {[15,16][12,10,9][4,4,4,5,4,5,5]} & 18 & \left(^{*}, 3\right) \\ 31 & {[15,16][12,11,8][4,4,4,5,4,5,5]} & 14 & \left(^{*}, 3\right) \\ 32 & {[16,16][12,11,9][2,5,5,5,5,5,5]} & 14 & \left(^{*}, 6\right) \\ 32 & {[16,16][12,10,10][2,5,5,5,5,5,5]} & 16 & \left(^{*}, 6\right) \\ 32 & {[16,16][11,11,10][5,4,4,5,4,5,5]} & 24 & \left(^{*}, 3\right) \\ 33 & {[15,18][12,12,9][3,5,5,5,5,5,5]} & 14 & \left(^{*}, 6\right) \\ 33 & {[17,16][13,11,9][3,5,5,5,5,5,5]} & 16 & \left(^{*}, 6\right) \\ 33 & {[15,18][12,11,10][3,5,5,5,5,5,5]} & 18 & \left(^{*}, 6\right) \\ 33 & {[17,16][12,12,9][3,5,5,5,5,5,5]} & 18 & \left(^{*}, 6\right) \\ 33 & {[15,18][11,11,11][3,5,5,5,5,5,5]} & 20 & \left(^{*}, 6\right) \\ 33 & {[17,16][13,10,10][3,5,5,5,5,5,5]} & 18 & \left(^{*}, 6\right) \\ 33 & {[17,16][12,11,10][3,5,5,5,5,5,5]} & 22 & \left(^{*}, 6\right) \\ 33 & {[17,16][11,11,11][3,5,5,5,5,5,5]} & 24 & \left(^{*}, 6\right)\end{array}$


Table E-4: Admissible multiplicity vectors with $(*, 6)$ symmetry for $33<n<40$.

$\begin{array}{lcc}n & \text { multiplicity vector } & \text { rid. index } \\ 34 & {[18,16][12,11,11][4,5,5,5,5,5,5]} & 26 \\ 34 & {[16,18][12,11,11][4,5,5,5,5,5,5]} & 26 \\ 34 & {[18,16][12,12,10][4,5,5,5,5,5,5]} & 24 \\ 34 & {[16,18][12,12,10][4,5,5,5,5,5,5]} & 24 \\ 34 & {[16,18][13,11,10][4,5,5,5,5,5,5]} & 22 \\ 34 & {[16,18][13,12,9][4,5,5,5,5,5,5]} & 18 \\ 35 & {[17,18][12,12,11][5,5,5,5,5,5,5]} & 30 \\ 35 & {[17,18][13,11,11][5,5,5,5,5,5,5]} & 28 \\ 35 & {[17,18][13,12,10][5,5,5,5,5,5,5]} & 26 \\ 35 & {[17,18][13,13,9][5,5,5,5,5,5,5]} & 20 \\ 35 & {[15,20][12,12,11][5,5,5,5,5,5,5]} & 18 \\ 36 & {[18,18][12,12,12][6,5,5,5,5,5,5]} & 32 \\ 36 & {[16,20][12,12,12][6,5,5,5,5,5,5]} & 24 \\ 36 & {[16,20][13,12,11][6,5,5,5,5,5,5]} & 22 \\ 36 & {[16,20][13,13,10][6,5,5,5,5,5,5]} & 18 \\ 37 & {[17,20][13,12,12][7,5,5,5,5,5,5]} & 26 \\ 37 & {[17,20][13,13,11][7,5,5,5,5,5,5]} & 24 \\ 38 & {[18,20][14,13,11][2,6,6,6,6,6,6]} & 16 \\ 38 & {[18,20][14,12,12][2,6,6,6,6,6,6]} & 18 \\ 38 & {[18,20][13,13,12][2,6,6,6,6,6,6]} & 20 \\ 38 & {[20,18][14,13,11][2,6,6,6,6,6,6]} & 16 \\ 38 & {[20,18][14,12,12][2,6,6,6,6,6,6]} & 18 \\ 38 & {[20,18][13,13,12][2,6,6,6,6,6,6]} & 20 \\ 39 & {[19,20][15,14,10][3,6,6,6,6,6,6]} & 16 \\ 39 & {[19,20][15,13,11][3,6,6,6,6,6,6]} & 22 \\ 39 & {[19,20][14,14,11][3,6,6,6,6,6,6]} & 24 \\ 39 & {[19,20][15,12,12][3,6,6,6,6,6,6]} & 24 \\ 39 & {[19,20][14,13,12][3,6,6,6,6,6,6]} & 28 \\ 39 & {[19,20][13,13,13][3,6,6,6,6,6,6]} & 30 \\ 39 & {[21,18][15,13,11][3,6,6,6,6,6,6]} & 18 \\ 39 & {[21,18][14,14,11][3,6,6,6,6,6,6]} & 20 \\ 39 & {[21,18][15,12,12][3,6,6,6,6,6,6]} & 20 \\ 39 & {[21,18][14,13,12][3,6,6,6,6,6,6]} & 24 \\ 39 & {[21,18][13,13,13][3,6,6,6,6,6,6]} & 26 \\ & & \end{array}$


Appendix F. Admissible multiplicity vectors for $p=7$

Table F-1: $1<n<26$.

$\begin{array}{rrrr} & \text { multiplicity vector } & \text { rid. index } & \text { symm type } \\ 13 & {[7,6][4,3,6][2,2,2,2,2,2,1]} & 0 & (1, *) \\ 14 & {[6,8][5,3,6][2,2,2,2,2,2,2]} & 0 & (1, *) \\ 15 & {[7,8][4,4,7][3,2,2,2,2,2,2]} & 0 & (1, *) \\ 16 & {[8,8][5,4,7][3,3,2,2,2,2,2]} & 2 & (1, *) \\ 19 & {[9,10][6,5,8][3,3,3,3,3,2,2]} & 4 & (1, *) \\ 19 & {[9,10][6,5,8][3,3,3,3,3,3,1]} & 2 & (1, *) \\ 20 & {[10,10][6,7,7][3,3,3,3,3,3,2]} & 10 & (2, *) \\ 20 & {[10,10][7,5,8][3,3,3,3,3,3,2]} & 6 & (1, *) \\ 20 & {[10,10][5,6,9][3,3,3,3,3,3,2]} & 2 & (1, *) \\ 21 & {[11,10][7,7,7][3,3,3,3,3,3,3]} & 12 & (2, *) \\ 21 & {[11,10][6,6,9][3,3,3,3,3,3,3]} & 6 & (1, *) \\ 21 & {[11,10][6,6,9][4,3,3,3,3,3,2]} & 4 & (1, *) \\ 21 & {[9,12][6,6,9][3,3,3,3,3,3,3]} & 2 & (1, *) \\ 22 & {[10,12][7,6,9][4,3,3,3,3,3,3]} & 6 & (1, *) \\ 23 & {[11,12][7,8,8][4,4,3,3,3,3,3]} & 12 & (2, *) \\ 23 & {[11,12][8,6,9][4,4,3,3,3,3,3]} & 8 & (1, *) \\ 23 & {[11,12][6,7,10][4,4,3,3,3,3,3]} & 4 & (1, *) \\ 24 & {[12,12][8,8,8][4,4,4,3,3,3,3]} & 14 & (2, *) \\ 24 & {[12,12][8,8,8][4,4,4,4,3,3,2]} & 12 & (2, *) \\ 24 & {[12,12][7,7,10][4,4,4,3,3,3,3]} & 8 & (1, *) \\ 24 & {[12,12][7,7,10][4,4,4,4,3,2,2]} & 6 & (1, *) \\ 24 & {[12,12][7,7,10][5,4,3,3,3,3,3]} & 6 & (1, *) \\ 25 & {[13,12][7,9,9][4,4,4,4,3,3,3]} & 12 & (2, *) \\ 25 & {[13,12][8,7,10][4,4,4,4,3,3,3]} & 10 & (1, *) \\ 25 & {[13,12][8,7,10][4,4,4,4,4,3,2]} & 8 & (1, *) \\ 25 & {[13,12][8,7,10][4,4,4,4,4,4,1]} & 4 & (1, *)\end{array}$

Table F-2: Admissible multiplicity vectors with $(2, *)$ symmetry for $p=7$ for $25<n<31$.

$\begin{array}{rrc}n & \text { multiplicity vector } & \text { rid. index } \\ 26 & {[12,14][8,9,9][4,4,4,4,4,4,2]} & 12 \\ 26 & {[14,12][8,9,9][4,4,4,4,4,4,2]} & 12 \\ 26 & {[12,14][8,9,9][4,4,4,4,4,3,3]} & 14 \\ 26 & {[14,12][8,9,9][4,4,4,4,4,3,3]} & 14 \\ 27 & {[13,14][9,9,9][5,4,4,4,4,4,2]} & 14 \\ 27 & {[13,14][9,9,9][5,4,4,4,4,3,3]} & 16 \\ 27 & {[13,14][7,10,10][4,4,4,4,4,4,3]} & 12 \\ 27 & {[13,14][9,9,9][4,4,4,4,4,4,3]} & 18 \\ 28 & {[14,14][10,9,9][4,4,4,4,4,4,4]} & 20 \\ 28 & {[14,14][8,10,10][4,4,4,4,4,4,4]} & 18 \\ 28 & {[14,14][8,10,10][5,4,4,4,4,4,3]} & 16\end{array}$




$\begin{array}{lcc}28 & {[14,14][8,10,10][5,5,4,4,4,3,3]} & 14 \\ 28 & {[14,14][8,10,10][5,5,4,4,4,4,2]} & 12 \\ 29 & {[15,14][9,10,10][5,4,4,4,4,4,4]} & 20 \\ 29 & {[15,14][9,10,10][5,5,4,4,4,4,3]} & 18 \\ 29 & {[15,14][9,10,10][5,5,5,4,4,3,3]} & 16 \\ 29 & {[13,16][9,10,10][5,4,4,4,4,4,4]} & 16 \\ 29 & {[15,14][9,10,10][5,5,5,4,4,4,2]} & 14 \\ 29 & {[15,14][9,10,10][5,5,5,5,4,4,4]} & 14 \\ 30 & {[14,16][10,10,10][5,5,4,4,4,4,4]} & 20 \\ 30 & {[14,16][10,10,10][6,4,4,4,4,4,3]} & 18 \\ 30 & {[14,16][8,11,11][5,5,4,4,4,4,4]} & 14 \\ 30 & {[16,14][8,11,11][5,5,5,4,4,4,4]} & 14\end{array}$

Appendix G. Admissible orthogonal multiplicity vectors

Table G-1: $p \neq 2,3,7$ and $6<n<19$.

$\begin{array}{rccc}n & \text { multiplicity vector } & \text { rid. index } & \text { symm. type } \\ 7 & {[3,4][1,3,3][1,1,1,1,1,1,1]} & 0 & (2,6) \\ 7 & {[3,4][3,2,2][1,1,1,1,1,1,1]} & 2 & (2,6) \\ 8 & {[4,4][2,3,3][2,1,1,1,1,1,1]} & 2 & (2,6) \\ 9 & {[5,4][3,3,3][1,1,1,2,2,1,1]} & 2 & (2,2) \\ 11 & {[5,6][3,4,4][1,1,2,2,2,2,1]} & 2 & (2,2) \\ 12 & {[6,6][4,4,4][0,2,2,2,2,2,2]} & 2 & (2,6) \\ 12 & {[6,6][4,4,4][2,1,2,2,2,2,1]} & 4 & (2,2) \\ 13 & {[7,6][5,4,4][1,2,2,2,2,2,2]} & 4 & (2,6) \\ 13 & {[7,6][3,5,5][1,2,2,2,2,2,2]} & 2 & (2,6) \\ 14 & {[8,6][4,5,5][2,2,2,2,2,2,2]} & 4 & (2,6) \\ 14 & {[6,8][5,4,4][2,2,2,2,2,2,2]} & 4 & (2,6) \\ 15 & {[7,8][5,5,5][3,2,2,2,2,2,2]} & 6 & (2,6) \\ 15 & {[7,8][5,5,5][1,2,2,3,3,2,2]} & 4 & (2,2) \\ 16 & {[8,8][6,5,5][2,2,2,3,3,2,2]} & 6 & (2,2) \\ 16 & {[8,8][4,6,6][2,2,2,3,3,2,2]} & 4 & (2,2) \\ 17 & {[9,8][5,6,6][3,2,2,3,3,2,2]} & 6 & (2,2) \\ 17 & {[9,8][5,6,6][1,2,3,3,3,3,2]} & 4 & (2,2) \\ 18 & {[8,10][6,6,6][2,2,3,3,3,3,2]} & 6 & (2,2) \\ 18 & {[10,8][6,6,6][2,2,3,3,3,3,2]} & 6 & (2,2)\end{array}$

Table G-2: Admissible orthogonal multiplicity vectors with $(2,6)$ symmetry for $p \neq 2,3,7$ and $18<n<32$.

$\begin{array}{rcc}n & \text { multiplicity vector } & \text { rid. index } \\ 19 & {[9,10][5,7,7][1,3,3,3,3,3,3]} & 4 \\ 19 & {[9,10][7,6,6][1,3,3,3,3,3,3]} & 6 \\ 20 & {[10,10][6,7,7][2,3,3,3,3,3,3]} & 10 \\ 20 & {[10,10][8,6,6][2,3,3,3,3,3,3]} & 8\end{array}$




$\begin{array}{lrc}21 & {[9,12][5,8,8][3,3,3,3,3,3,3]} & 6 \\ 21 & {[11,10][7,7,7][3,3,3,3,3,3,3]} & 12 \\ 22 & {[10,12][6,8,8][4,3,3,3,3,3,3]} & 8 \\ 22 & {[12,10][6,8,8][4,3,3,3,3,3,3]} & 8 \\ 22 & {[10,12][8,7,7][4,3,3,3,3,3,3]} & 10 \\ 23 & {[11,12][7,8,8][5,3,3,3,3,3,3]} & 10 \\ 25 & {[13,12][7,9,9][1,4,4,4,4,4,4]} & 6 \\ 25 & {[13,12][9,8,8][1,4,4,4,4,4,4]} & 8 \\ 26 & {[12,14][10,8,8][2,4,4,4,4,4,4]} & 10 \\ 26 & {[14,12][10,8,8][2,4,4,4,4,4,4]} & 10 \\ 26 & {[12,14][8,9,9][2,4,4,4,4,4,4]} & 12 \\ 26 & {[14,12][8,9,9][2,4,4,4,4,4,4]} & 12 \\ 27 & {[15,12][7,10,10][3,4,4,4,4,4,4]} & 8 \\ 27 & {[13,14][7,10,10][3,4,4,4,4,4,4]} & 12 \\ 27 & {[13,14][11,8,8][3,4,4,4,4,4,4]} & 12 \\ 27 & {[15,12][9,9,9][3,4,4,4,4,4,4]} & 14 \\ 27 & {[13,14][9,9,9][3,4,4,4,4,4,4]} & 18 \\ 28 & {[12,16][8,10,10][4,4,4,4,4,4,4]} & 10 \\ 28 & {[16,12][8,10,10][4,4,4,4,4,4,4]} & 10 \\ 28 & {[12,16][10,9,9][4,4,4,4,4,4,4]} & 12 \\ 28 & {[14,14][8,10,10][4,4,4,4,4,4,4]} & 18 \\ 28 & {[14,14][10,9,9][4,4,4,4,4,4,4]} & 20 \\ 29 & {[15,14][7,11,11][5,4,4,4,4,4,4]} & 10 \\ 29 & {[13,16][11,9,9][5,4,4,4,4,4,4]} & 14 \\ 29 & {[13,16][9,10,10][5,4,4,4,4,4,4]} & 16 \\ 29 & {[15,14][9,10,10][5,4,4,4,4,4,4]} & 20 \\ 30 & {[14,16][8,11,11][6,4,4,4,4,4,4]} & 12 \\ 30 & {[16,14][8,11,11][6,4,4,4,4,4,4]} & 12 \\ 30 & {[14,16][10,10,10][6,4,4,4,4,4,4]} & 18 \\ 31 & {[15,16][11,10,10][1,5,5,5,5,5,5]} & 10 \\ 31 & {[15,16][9,11,11][7,4,4,4,4,4,4]} & 14 \\ & & \end{array}$

Table G-3: $p=3$ and $6<n<19$.

$\begin{array}{rrrr}n & \text { multiplicity vector } & \text { rid. index } & \text { symm. typ } \\ 7 & {[3,4][3,3,1][1,1,1,1,1,1,1]} & 0 & (*, 6) \\ 7 & {[3,4][3,2,2][1,1,1,1,1,1,1]} & 2 & (*, 6) \\ 9 & {[5,4][3,3,3][1,1,1,2,2,1,1]} & 2 & (*, 2) \\ 12 & {[6,6][4,4,4][0,2,2,2,2,2,2]} & 2 & (*, 6) \\ 12 & {[6,6][4,4,4][2,1,2,2,2,2,1]} & 4 & (*, 2) \\ 13 & {[7,6][5,4,4][1,2,2,2,2,2,2]} & 4 & (*, 6) \\ 13 & {[7,6][5,5,3][2,1,2,2,2,2,1]} & 2 & (*, 6) \\ 15 & {[7,8][5,5,5][3,2,2,2,2,2,2]} & 6 & (*, 6) \\ 15 & {[7,8][5,5,5][1,2,2,3,3,2,2]} & 4 & (*, 2) \\ 16 & {[8,8][6,6,4][2,2,2,3,3,2,2]} & 4 & (*, 2) \\ 16 & {[8,8][6,5,5][2,2,2,3,3,2,2]} & 6 & (*, 2) \\ 18 & {[8,10][6,6,6][2,2,3,3,3,3,2]} & 6 & (*, 2) \\ 18 & {[10,8][6,6,6][2,2,3,3,3,3,2]} & 6 & (*, 2)\end{array}$

Jordan shape 
Table G-4: Admissible orthogonal multiplicity vectors of $(*, 6)$ symmetry for $p=3$ and $18<n<32$.

$n$

19

19

20

20

21

21

22

22

25

25

26

26

26

26

27

27

27

27

28

28

28

28

29

29

30

31

31 multiplicity vector

$[9,10][7,7,5][1,3,3,3,3,3,3]$

$[9,10][7,6,6][1,3,3,3,3,3,3]$

$[10,10][8,6,6][2,3,3,3,3,3,3]$

$[10,10][8,7,5][2,3,3,3,3,3,3]$

$[9,12][7,7,7][3,3,3,3,3,3,3]$

$[11,10][7,7,7][3,3,3,3,3,3,3]$

$[10,12][8,8,6][4,3,3,3,3,3,3]$

$[10,12][8,7,7][4,3,3,3,3,3,3]$

$[13,12][9,9,7][1,4,4,4,4,4,4]$

$[13,12][9,8,8][1,4,4,4,4,4,4]$

$[12,14][10,9,7][2,4,4,4,4,4,4]$

$[14,16][10,9,7][2,4,4,4,4,4,4]$

$[12,14][10,8,8][2,4,4,4,4,4,4]$

$[14,12][10,8,8][2,4,4,4,4,4,4]$

$[13,14][11,9,7][3,4,4,4,4,4,4]$

$[13,14][11,8,8][3,4,4,4,4,4,4]$

$[15,12][9,9,9][3,4,4,4,4,4,4]$

$[13,14][9,9,9][3,4,4,4,4,4,4]$

$[12,16] \quad[10,10,8][4,4,4,4,4,4,4]$

$[12,16] \quad[10,9,9][4,4,4,4,4,4,4]$

$[14,14][10,10,8][4,4,4,4,4,4,4]$

$[14,14] \quad[10,9,9][4,4,4,4,4,4,4]$

$[13,16][11,10,8][5,4,4,4,4,4,4]$

$[13,16][11,9,9][5,4,4,4,4,4,4]$

$[14,16][10,10,10][6,4,4,4,4,4,4]$

$[15,16][11,11,9][1,5,5,5,5,5,5]$

$[15,16][11,10,10][1,5,5,5,5,5,5]$ rid. index Jordan shape

4

$\left(5 J_{3}, 2 J_{2}\right)$

$\left(6 J_{3}, J_{1}\right)$

$\left(6 J_{3}, 2 J_{1}\right)$

$\left(5 J_{3}, 2 J_{2}, J_{1}\right)$

$\left(7 J_{3}\right)$

$\left(7 J_{3}\right)$

$\left(6 J_{3}, 2 J_{2}\right)$

$\left(7 J_{3}, J_{1}\right)$

$\left(7 J_{3}, 2 J_{2}\right)$

$\left(8 J_{3}, J_{1}\right)$

$\left(7 J_{3}, 2 J_{2}, J_{1}\right)$

$\left(7 J_{3}, 2 J_{2}, J_{1}\right)$

$\left(8 J_{3}, 2 J_{1}\right)$

$\left(8 J_{3}, 2 J_{1}\right)$

$\left(7 J_{3}, 2 J_{2}, 2 J_{1}\right)$

$\left(8 J_{3}, 3 J_{1}\right)$

$\left(9 J_{3}\right)$

$\left(9 J_{3}\right)$

$\left(8 J_{3}, 2 J_{2}\right)$

$\left(9 J_{3}, J_{1}\right)$

$\left(8 J_{3}, 2 J_{2}\right)$

$\left(9 J_{3}, J_{1}\right)$

$\left(8 J_{3}, 2 J_{2}, J_{1}\right)$

$\left(9 J_{3}, 2 J_{2}\right)$

$\left(10 J_{3}\right)$

$\left(9 J_{3}, 2 J_{2}\right)$

$\left(10 J_{3}, J_{1}\right)$

Table G-5: $p=7$ and $6<n<20$.

$n$

7

7

8

12

12

14

14

15

17

19

19

19

19 multiplicity vector

$[3,4][1,3,3][1,1,1,1,1,1,1]$

$[3,4][3,2,2][1,1,1,1,1,1,1]$

$[4,4][2,3,3][2,1,1,1,1,1,1]$

$[6,6][4,4,4][2,2,2,2,2,2,0]$

$[6,6][4,4,4][2,2,2,2,2,1,1]$

$[6,8][4,5,5][2,2,2,2,2,2,2]$

$[8,6][4,5,5][2,2,2,2,2,2,2]$

$[7,8][5,5,5][3,2,2,2,2,2,2]$

$[9,8][5,6,6][3,3,3,2,2,2,2]$

$[9,10][5,7,7][3,3,3,3,3,3,1]$

$[9,10][5,7,7][3,3,3,3,3,2,2]$

$[9,10][7,6,6][3,3,3,3,3,2,2]$

$[9,10][7,6,6][3,3,3,3,3,3,1]$ rid. index Jordan shape

0

2

$J_{7}$

$J_{7}$

$\left(J_{7}, J_{1}\right)$

$\left(2 J_{6}\right)$

$\left(J_{7}, J_{5}\right)$

$\left(2 J_{7}\right)$

$\left(2 J_{7}\right)$

$\left(2 J_{7}, J_{1}\right)$

$\left(2 J_{7}, J_{3}\right)$

$\left(J_{7}, 2 J_{6}\right)$

$\left(2 J_{7}, J_{5}\right)$

$\left(2 J_{7}, J_{5}\right)$

$\left(J_{7}, 2 J_{6}\right)$ 
Acknowledgement The authors are grateful to the referee for many useful comments, and for checking the tables.

\section{References}

1. J. H. Conway, R. T. Curtis, S. P. Norton, R. A. Parker and R. A. Wilson, ATLAS of finite groups (Clarendon Press, Oxford, 1985). 41, 42, 53,54

2. C. Jansen, K. Lux, R. A. Parker and R. A. Wilson, An Atlas of Brauer characters (Clarendon Press, Oxford, 1995). 41, 42, 54

3. J. M. Cohen, 'On non-Hurwitz groups and non-congruence subgroups of the modular group', Glasgow Math. J. 22 (1981) 1-7. 22

4. M. D. E. CONDER, 'Generators for alternating and symmetric groups', $J$. London Math. Soc. (2) 22 (1980) 75-86. 21

5. M. D. E. Conder, 'Hurwitz groups: a brief survey', Bull. Amer. Math. Soc. 23 (1990) 359-370.

6. L. Di Martino, Ch. Tamburini and A. Zalesski, 'Hurwitz groups of small rank', Comm. Algebra 28 (2000) 5383-5404. 21, 22, 23, 24, 25, 47, 53, 54

7. W. FEIT, The representation theory of finite groups (North-Holland, Amsterdam, 1982). 38, 41

8. B. Huppert, Endliche Gruppen I (Springer, Berlin/Heidelberg, 1967). 39

9. J. Humphreys, Conjugacy classes in semisimple algebraic groups (Amer. Math. Soc., Providence, RI, 1995). 34

10. G. Jones, 'Ree groups and Riemann surfaces', J. Algebra 165(1994), 41-62. 21,46

11. P. Kleidman and M. W. LieBeCK, Subgroup structure of classical groups (Cambridge Univ. Press, Cambridge 1990). 30, 59

12. A. Lucchini, M. C. Tamburini and J. Wilson, Hurwitz groups of large rank, J. London Math. Soc. (2) 61(2000) 81-92. 21, 23

13. A. Lucchini and M. C. TAmburini, 'Classical groups of large rank as Hurwitz groups', J. Algebra 219 (1999) 531-546. 21

14. A. M. Macbeath, Generators of the linear fractional groups, Proc. Symp. Pure Math. 12 (1969) 14-32. 39

15. G. Malle, 'Hurwitz groups and $G_{2}(q)$ ', Canad. Math. Bull. 33 (1990) 349356. 21,46

16. G. MALle, 'Small rank exceptional Hurwitz groups', Groups of Lie type and their geometries, London Math. Soc. Lecture Notes 207 (Cambridge Univ. Press, Cambridge, 1995) 173-183. 21, 23, 46

17. C. T. Simpson, 'Products of matrices', Differential geometry, global analysis and topology, Canad. Math. Soc. Conf. Proc. 12 (Amer. Math. Soc., Providence, RI, 1991) 157-185. 29

18. R. Steinberg, 'Lectures on Chevalley groups', Yale Univ., 1967. 40, 43, 54

19. L. Scotт, Matrices and cohomology, Ann. of Math. 105 (1987) 473-492. 24, 27 
20. T. Springer and R. Steinberg, 'Conjugacy classes', 'Seminar on algebraic groups and related finite groups, Lect. Notes in Math. 131 (Springer, Berlin, 1970). 38

21. K. Strambach and H. Völklein, 'On linearly rigid tuples', J. Reine Angew. Math. 510 (1999) 57-62. 29

22. Ch. Tamburini and $\mathrm{S}$. Vassallo, '(2,3)-generazione di $\mathrm{SL}(4, q)$ in caracteristica dispari e problemi collegati', Boll. Un. Mat. Ital. (7) (1994) 121-134. 24

23. Ch. Tamburini and $M$. Vsemirnov, 'Hurwitz groups and Hurwitz generation', Handbook of algebra, vol.4 (Elsevier, 2006) 385-426. 21, 22, 46

24. Ch. Tamburini and M. Vsemirnov, 'Irreducible (2,3,7)-subgroups of $\mathrm{PGL}_{n}(F), n \leqslant 7$ ', J. Algebra 300 (2006) 339-362. 25

25. M. Chiara Tamburini and A.E. Zalesski, 'Classical groups in dimension 5 which are Hurwitz', Finite groups 2003, Proc. Gainesville Conf. on Finite Groups, March 6-12, 2003 (ed. C. Y. Ho, P. Sin, P. H. Tiep and A. Turull; De Gruyter, Berlin, 2004) 363-372. 25

26. M. Vsemirnov, 'Hurwitz groups of intermediate rank', LMS J. Comput. Math. 7 (2004) 300-336. 21, 23

27. R. Wilson, 'The Monster is a Hurwitz group', J. Group Theory 4 (2001) 367-374. 21

28. Yongzhong Sun, 'On the $(2,3,7)$-Generation of some special linear groups', Comm. Algebra 34 (2006) 51-74. 23

29. A. ZALESSKI, 'Minimal polynomials and eigenvalues of $p$-elements in representations of groups with a cyclic Sylow p-subgroup', J. London Math. Soc. 59 (1999) 845-866. 41

R. Vincent Robert.Vincent@uea.ac.uk

A.E. Zalesski a.zalesskii@uea.ac.uk

School of Mathematics

University of East Anglia

Norwich NR47TJ

United Kingdom 